ORGANISATION EUROPEEENNE POUR LA RECHERCHE NUCLËAIRE CERN european organization for nuClear research

\title{
INTRODUCTION TO RELIABILITY THEORY
}

B. Schorr

Lectures given in the

Academic Training Programme of CERN 1973-1974

G E N E V A 


\section{(C) Copyright CERN, Genève, 1974}

Propriété littéraire et scientifique réservée pour tous les pays du monde. Ce document ne peut être reproduit ou traduit en tout ou en partie sans l'autorisation écrite du Directeur général du CERN, titulaire du droit d'auteur. Dans les cas appropriés, et s'il s'agit d'utiliser le document à des fins non commerciales, cette autorisation sera volontiers accordée.

Le CERN ne revendique pas la propriété des inventions brevetables et dessins ou modèles susceptibles de dépôt qui pourraient être décrits dans le présent document; ceux-ci peuvent être librement utilisés par les instituts de recherche, les industriels et autres intéressés. Cependant, le CERN se réserve le droit de s'opposer à toute revendication qu'un usager pourrait faire de la propriété scientifique ou industrielle de toute invention et tout dessin ou modèle décrits dans le présent document.
Literary and scientific copyrights reserved in all countries of the world. This report, or any part of it, may not be reprinted or translated without written permission of the ccpyright holder, the Director-General of CERN. However, permission will be freely granted for appropriate non-commercial use. If any patentable invention or registrable design is described in the report, CERN makes no claim to property rights in it but offers it for the free use of research institutions, manufacturers and others. CERN, however, may oppose any attempt by a user to claim any proprietary or patent rights in such inventions or designs as may be described in the present document. 


\section{ORGANISATION EUROPÉENNE POUR LA RECHERCHE NUCLĒAIRE \\ CERN european organization for nUCLEAR RESEARCH}

\section{INTRODUCTION TO RELIABILITY THEORY}

B. Schorr

Lectures given in the

Academic Training Programme of CERN 1973-1974

G E N E V A 
CERN - Service d'Information scientifique - H/581 - 2000 - septembre 1974 
This report consists of lecture notes of a series of introductory lectures on reliability given at CERN in the Spring of 1974. It covers unrepairable and repairable systems. In addition, some optimization problems are discussed. Two major examples, one from the airplane industry, the other from the computer industry, illustrate the theory. In an Appendix, basic concepts of probability and statistics are summarized to help the reader understand the report. The report is intended for engineers and physicists who have to deal with design and/or maintenance of complex systems. 


\section{CONTENTS}

\begin{tabular}{|c|c|c|}
\hline & & Page \\
\hline INTRODUCTION & & 1 \\
\hline CHAPTER I & UNREPAIRABLE SYSTEMS & 5 \\
\hline & 1. DEFINITION OF RELIABILITY CHARACTERISTICS & 5 \\
\hline & 2. TYPICAL FAILURE DISTRIBUTIONS & 8 \\
\hline & 3. IFR FAILURE DISTRIBUTIONS & 11 \\
\hline & 4. SERIES CONNECTIONS & 12 \\
\hline & 5. PARALLEL CONNECTIONS (HOT REDUNDANCIES) & 16 \\
\hline & 6. PARALLEL CONNECTIONS (COLD REDUNDANCIES) & 19 \\
\hline & 7. THE FUEL SUPPLY SYSTEM OF AN AIRLINER & 24 \\
\hline CHAPTER II & REPAIRABLE SYSTEMS & 35 \\
\hline & 1. DEFINITION OF RELIABILITY CHARACTERISTICS & 35 \\
\hline & 2. SERIES CONNECTIONS & 41 \\
\hline & 3. COMPUTER PROGRAM TO MONITOR THE MAINTENANCE ON THE CDC 6600 & 49 \\
\hline CHAPTER III & OPTIMIZATION PROBLEMS & 51 \\
\hline & 1. INTRODUCTORY REMARKS & 51 \\
\hline & 2. OPTIMIZATION & 51 \\
\hline & 3. REDUNDANCY OPTIMIZATION & 53 \\
\hline & 4. NUMBER OF SPARE PARTS & 57 \\
\hline & 4.1 Average number of spare parts & 57 \\
\hline & 4.2 Optimum number of spare parts for a series connection & 58 \\
\hline & 5. OPTIMUM TIME INTERVAL FOR AGE REPLACEMENT & 60 \\
\hline APPENDIX & & 63 \\
\hline REFERENCES & & 81 \\
\hline
\end{tabular}




\section{INTRODUCTION}

One of the things which make life difficult is the fact that we often cannot rely on our fellow-men or even on ourselves. We expect from these people, or from ourselves, certain activities to be done in a given time, and when this time is over and things are not done as we expected, we are, at the least, disappointed. In the case where we observe from time to time such behaviour on the part of the same individuals, we say that these people are not reliable. That means we cannot predict with certainty that they will do their job properly next time. This problem certainly is as old as mankind, as the story of Adam and Eve clearly shows. In order to help solve the problem of creating reliable individuals education is considered to be one of the means.

When men began to use simple tools like knives, hatches, wedges and so on, they also had to rely on them, i.e. they had to make sure that the tools were functioning when they were to be used. To make these simple tools reliable, it was essentially sufficient to make them fairly strong and solid. However, as over thousands of years tools gradually became sophisticated, both in their structure as well as with respect to their mission, the solution of the problem became more difficult. Apparently, the original method of making the elements of the tools "strong" and "solid" enough could not be used any longer in a straightforward way. In other words, compromises had to be made: they had to be not too "strong" and not too "solid" but still "strong" and "solid" enough so that the tools worked reliably. When, however, people tried to find solutions on the basis of compromises, it turned out that a clear definition of what is meant by reliability of a tool was needed in order to have the possibility of comparing different designs with respect to that characteristic. This finally led to a definition which assigns to a tool a quantitative measure, a number, to describe its reliability, as we shall see later.

From now on we will always replace the word tool by the more general expression system. The problem of making a system reliable is basically an engineering problem. But when the quantitative reliability concept was introduced it also became a mathematical problem, at least to some extent. Therefore it is not surprising that nowadays there is a complete branch of its own in mathematics which is called reliability mathematics. First steps in the development and application of the concept of reliability were made in the nineteen thirties in the field of machine maintenance. The main impulse, however, in engineering as well as in mathematics, grew out of the necessities and the experiences of the Second World War. Finally, it was the exploding growth of aeronautics and astronautics, correlated with the complexity of systems, which stimulated, in a fascinating way, research and application of reliability theory.

Let us briefly come back to what we said about how to create reliable fellow-men. It is education which is believed to help in solving this problem. Being fairly broadminded 
we may also say that systems ought to be "educated" to reliability in the sense that, already beginning with the design and then during the development and the production, the reliability of a system must be built in. The work which has to be done to "educate" in this way a system to reliability is simply called reliability work. This kind of work should already begin in the projection phase of a system and should continue practically till the end of the life of the system. In the beginning, reasonable reliability specifications have to be formulated. In the design phase the system has to be designed such that it will meet the reliability specifications. In the development and the production phase care has to be taken that the system finally will have the required reliability. During operation the necessary maintenance and repair activities have to be done to keep the system on the required reliability level, and finally it is important for later designs and developments to keep records of the system failures during its whole lifetime.

The whole reliability work obviously demonstrates that not only do the designers of a system have to be familiar with the concepts of reliability theory and their practical application, but that many other people should know at least the basic concepts of it, beginning from the individuals who order a system to those who are responsibie for its development, production, maintenance and repair.

Let us briefly discuss an example which demonstrates how useful reliability work on a system can be. We consider a typical analysis of the direct operational cost of a modern short-range airliner. Figure 1 shows that the maintenance and repair costs are about $20 \%$ of the total cost. In addition we have about $3 \%$ of the amortization cost which comes from the spare parts side. The maintenance and repair costs are in the order of magnitude of fuel and oil costs. Therefore, from the point of view of cost, it is equally important to reduce maintenance and repair costs as well as fuel and oil costs, disregarding safety, availabilitiy and other aspects which have to be considered for an airliner.

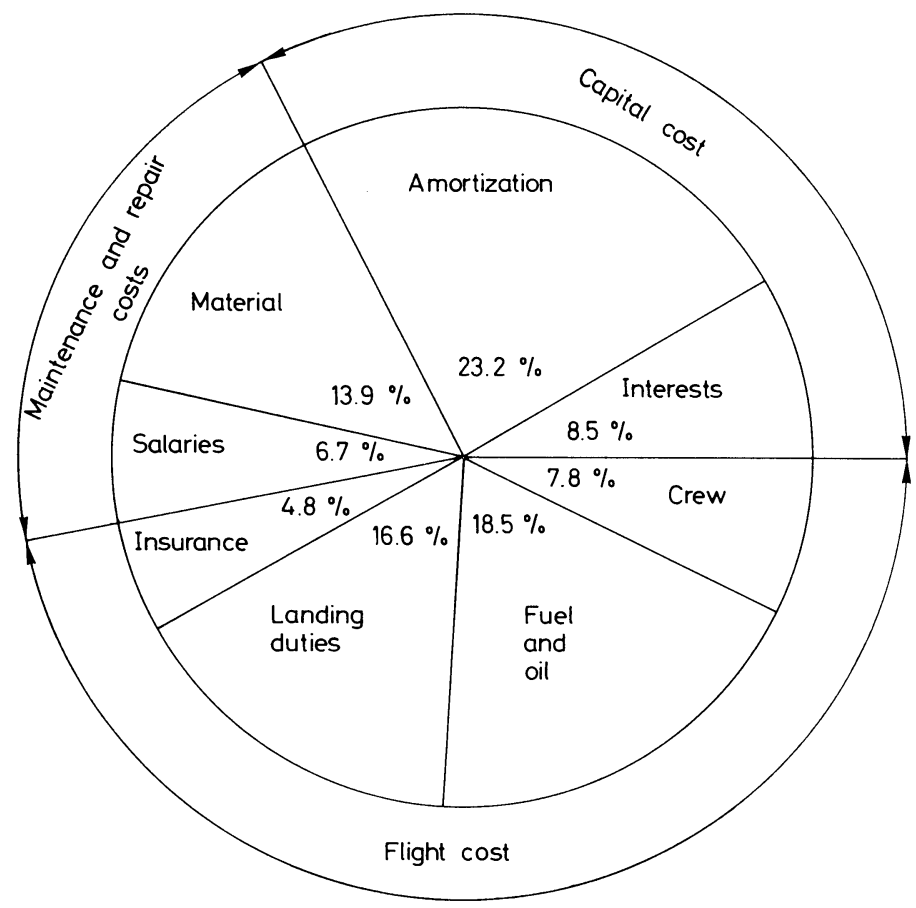

Fig. 1 Operational cost of an airliner [taken from Brink et al. 1)] 
The costs for maintenance and repair result from a lot of separate costs. Therefore, the total cost for maintenance and repair has to be analysed. Such an analysis essentially reveals that maintenance and repair costs are reduced if, for instance, we have

- a small number of components to reduce failures,

- low cost of components,

- long operating times of components, i.e. low failure rates,

- low number of man-hours for scheduled maintenance, e.g. quick exchange of spare parts,

- and so on.

The reliability work done in the design phase of the airplane will particularly take care of the points mentioned.

Let us have a look at a few actual figures in our example. The total cost for maintenance and repair is about $\$ 400$ per flight hour. The amount for scheduled inspections is roughly $15 \%$ of the total maintenance cost. A reduction of, for instance, $10 \%$ of the cost for these inspections means a saving of $\$ 6$ per flight hour and, with about 3000 flight hours per year, we have a yearly saving of $\$ 18,000$ per aircraft.

Furthermore, the mean time between two defects or two unscheduled repairs lies between three and six hours. In our example the average cost for repairing a defect is $\$ 660$. That means, that an increase of the mean time between two defects of again $10 \%$ would reduce costs by $\$ 10$ to $\$ 20$ per flight hour or by $\$ 48,000$ to $\$ 78,000$ per year and aircraft.

Naturally, we cannot drastically reduce costs for maintenance, repair, availability, safety and so on, without increasing costs for development and production. But it is obviously reasonable and promising to work on this problem in order to find an acceptable compromise between development and production costs on the one hand and maintenance and repair costs, for instance, on the other hand. The "best" compromise we can make is normally called the optimal solution of the problem. More about that will be discussed later in Chapter III on optimization.

We may say that the theory and application of reliability has basically four different aspects:

i) probabilistic: definition and prediction of reliability characteristics;

ii) statistical: data collection and evaluation;

iii) engineering: design, development, production, testing;

iv) managerial: responsibilities, communication, training.

In this report we will mainly cover probabilistic aspects and to some extent design aspects in reliability engineering. Statistical aspects will be touched on only occasionally and managerial aspects are not covered at all. The report discusses in an elementary way the basic concepts of reliability theory. It gives the main ideas of the field to interested engineers, physicists and mathematicians. It should, for instance, enable them to follow discussions where reliability specifications are treated for new systems. The reader should, after a careful study of the report, be able to read more advanced literature on the subject. 
At the end of the report there are references to books, handbooks and papers which cover all the aspects of reliability mentioned. Those readers who want to contact people who are working practically in the field of reliability may find them, for instance, in the airplane, electronics, reactor or computer industry. In the airplane industry, for instance, they have normally groups of specialized reliability engineers. There exist also short courses about the field given by industrial experts [for example: in Germany VDI, Lehrgang über Technische Wartbarkeit und Zuverlässigkeit, Verein Deutscher Ingenieure, Düsseldorf; and ASQ, Lehrgang über Technische Zuverlässigkeit, Deutsche Gesellschaft für Qualität, (ASQ), Frankfurt].

The mathematical prerequisites which are necessary to read this report are essentially familiarity with calculus, systems of ordinary differential equations with constant coefficients, and the basic concepts of probability and statistics. Since it is assumed that not every reader is familiar enough with probability and statistics, a very brief introduction to basic concepts of these fields is given in the Appendix.

The report is divided into three chapters. The first chapter covers unrepairable systems. The basic reliability characteristics like lifetime, reliability, failure distribution, failure rate, mean time to failure, etc., are introduced for unrepairable systems. Then, after a discussion of typical failure distributions, series and parallel connections of system components are studied. This chapter finishes with the discussion of the reliability of the fuel supply system of an airliner. This example is based on a reliability study which was made in the design phase of an airliner. It will demonstrate how, in a practical case, the concepts introduced in Chapter I are applied.

In the second chapter repairable systems are discussed. There, additional reliability characteristics like maintainability, availability, and so on, are introduced. A detailed discussion of series connections of repairable components follows. Parallel connections of repairable components are not discussed. The mathematical treatment of such systems is too involved for an introductory report. A reader who has studied the section on series connections of repairable systems will be able to understand the corresponding literature on parallel connections of repairable systems. Chapter II finishes with the discussion of an existing computer program for maintenance monitoring of a CDC 6600 computer.

The last chapter discusses optimization problems in the context of reliability theory. Special examples of redundancy optimization, the optimum number of spare parts, and the optimum choice of the time interval in an age replacement policy are discussed. 
CHAPTER I

\section{UNREPAIRABLE SYSTEMS}

\section{DEFINITION OF RELIABILITY CHARACTERISTICS}

We consider a system to be a unit, consisting of one or more components, which has to perform certain tasks. For instance, a computer, a car, an aircraft, a linear accelerator, and so on are systems. The components of a system may be systems themselves. In this case, we call them subsystems of the system. How a system is to be subdivided into subsystems, or sub-subsystems, and so on, is normally more a technical question or a question of convenience or of available detailed information about the subsystems.

For us a system is basically determined by its components, the logical connection of its components (series, parallel, etc.) and its tasks. In addition, we require to be given a criterion which uniquely defines whether the system is up or down. We shall only consider systems which are either up or down. We will not consider deteriorating systems. For such systems we refer to Chapter 7 of Kozlov and Ushakov ${ }^{2}$.

It is important to have a failure criterion, since it is not always a priori clear whether a system is up or down. This depends highly on the kind of tasks it has to perform. For instance, a car with failed lights must be considered as failed for public traffic, but it can still do the job on a private terrain during day-time.

We call a system (or a component) unrepairable if it cannot be repaired to perform its particular tasks after failure. This does not necessarily mean that the system cannot be repaired at all. It only means that failure of the system during operation leads to practically unrepairable consequences. For instance, an on-1ine computer controlling a process which does not allow any interruption must be considered as unrepairable in this context, even if it can be repaired later.

There exist many characteristics which are used to describe reliability aspects of systems and components. We shall discuss here the most common reliability characteristics. Others can be found in the references given at the end of this paper (see, for example, Ref. 2), or have to be invented in special practical problems as we will see later when we discuss practical examples. In principle, almost all of these characteristics are either probabilities or expected values of functions of random variables.

We begin with the definition of the reliability $\mathrm{R}$ of an unrepairable system. The basic idea to find a quantitative measure of what is called reliability of an apparatus in daily life is the following. We want to have a measure for the chances which are given that a system does not fail under given conditions during the time interval $[0, t]$. Under these circumstances we may consider the survival of a system to be a random event and the probability of survival is then a measure of the system's chances to survive. Therefore, the following definition of reliability of a system (or component) seems to be reasonable. 


\section{Definition 1}

The probability $R(t)$ that an unrepairable system performs without failure a specified function under given conditions for a specified time of length $t$ is called the reliability (or reliability function) of the system.

Let us first give an intuitive statistical interpretation of the reliability $R(t)$. Suppose we have a large number of the same systems which all started to run at $t=0$ and under the same conditions. After a time of length $t$ we calculate the relative frequency $\hat{R}(t)$ of the systems which have not failed. $\hat{R}(t)$ can then be considered as an estimate of the reliability $R(t)$. Conversely, if a system has, for instance, the reliability $R(t)=0.99$, this means that about $99 \%$ of a large number of the same sort of systems, all operating since $t=0$ and under the same conditions, will survive time $t$. However, we cannot say that a particular system out of such a lot will survive. But when we have two systems $S_{1}$ and $S_{2}$ with $R_{S_{1}}(t)>R_{S_{2}}(t)$ we will prefer $S_{1}$, since we have more confidence in $S_{1}$ than in $S_{2}$ because of the statistical interpretation given above. It may, however, turn out later that $S_{2}$ lives longer than $S_{1}$.

Looking at Definition 1 we observe that $R(t)$ is dependent not only on the time $t$, but also on the system itself (components, logical connection of the components, etc.), on the given conditions, and on the specified functions to be performed. In the present paper not all of these factors influencing $\mathrm{R}(\mathrm{t})$ will be discussed, since they mostly result from engineering problems to be considered separately for every specific system. We might mention that, for instance, humidity, heat, radiation, and so on, are important factors which have to be considered. Their influences on $R(t)$ are normally handled by using different tables $\left.{ }^{3}, 4\right)$. Or human factors as far as man-machine interaction is concerned have to be considered, and so $\left.\mathrm{on}^{3}, 5\right)$.

We shall confine our discussions mainly to the factors -- age, structure of the system, maintenance and repair -- and we will consider some mathematical models which describe the influence of these factors on the reliability $R(t)$. For this reason we first need some more definitions.

Let $\mathrm{T} \geq 0$ be a random variable describing the lifetime of a system $S$. In other words, $\mathrm{T}$ is the length of the time interval during which a system $\mathrm{S}$ operates without failure. We can then say that $R(t)$ is the probability that $T>t$ or

$$
R(t)=P(T>t)=1-F(t),
$$

where $F(t)=P(T \leq t)$ is the distribution function of the lifetime $T$. We say that $F(t)$ describes the failure distribution or the failure law of the system $S$. We suppose that the lifetime $T$ is always a continuous random variable with failure density function $f(t)=F^{\prime}(t)$. We can then introduce another reliability characteristic which is very important to describe the influence of ageing on the reliability of a system S.

\section{Definition 2}

Let $R(t)$ be the reliability and $f(t)$ the failure density function of an unrepairable system $S$. Then 


$$
\begin{gathered}
-7- \\
\lambda(t)=\frac{f(t)}{R(t)}
\end{gathered}
$$

is called the failure rate function of $\mathrm{S}$.

The failure rate function is a quantity which has the following intuitive meaning: $\lambda(t) \Delta t$ describes, to the first order of magnitude in $\Delta t$, the probability that the system $S$, having reached the age $t$, will fail during the interval $(t, t+\Delta t)$. Actuaries use this concept under the name "force of mortality" to calculate mortality tables. In reliability theory it is also called "hazard rate".

Practical experience shows that the function $\lambda(t)$ qualitatively behaves as shown in Fig. 2. Being very similar to the shape of a normal bath-tub, the curve in Fig. 2 is often called the bath-tub curve of reliability theory. The curve may be sub-divided into three different periods. Period I corresponds to so-called early failures or the debugging time. It represents the failure rate behaviour of a system caused by components which escaped quality control inspection. Suppose during period I the corresponding system was always repaired after failure so that the weak components were replaced by good ones. The system may then enter the period II, where the failure rate is more or less constant. This period is called the period of random failures. Such failures always occur because of some random influences which are unpredictable. We also call period II the useful lifetime of a system. It is normally considered to be the operating period of a system. After this period, wear out of the system will become significant and the system enters period III where wear out failures are dominant. When the system reaches period III special maintenance and repair operations have to start to keep the system out of this period and to bring it back to the random failure period. The period I should normally be considered as the running-in time of a system and its actual operation time should start when the failure rate is more or less constant. We shall therefore discuss primarily systems with failure rates which are either constant or monotonically increasing. We call a failure distribution with such a failure rate an IFR failure distribution (distribution with Increasing Failure Rate) or we say shortly the distribution is IFR. Occasionally we also say that the corresponding distribution function is IFR. Simply from the fact that a system has an IFR failure distribution many useful conclusions on its reliability $R(t)$ can already be drawn as we shall see later.

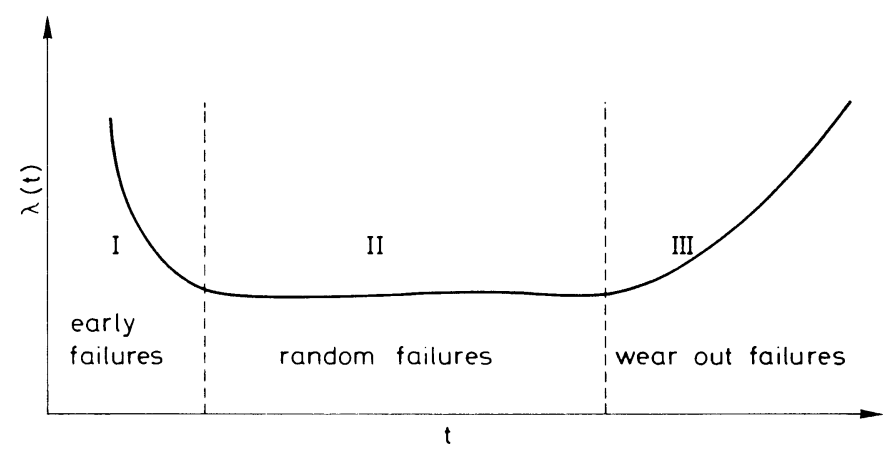

Fig. 2 Qualitative behaviour of the failure rate function $\lambda(t)$ 
From Eqs. (I.1) and (I.2) and the fact that $f(t)=F^{\prime}(t)=-R^{\prime}(t)$ we easily find that

$$
R^{\prime}(t)=-\lambda(t) R(t)
$$

Integration of this differential equation for $R(t)$, together with the obvious initial condition $R(0)=1$ (the system begins to run at $t=0$ ), yields

$$
R(t)=\exp \left[-\int_{0}^{t} \lambda(\tau) d \tau\right]
$$

Equation (I.4) shows that the knowledge of the failure rate is sufficient to determine the reliability function $R(t)$.

Taking into account Eqs. (I.1) to (I.4) together with $f(t)=F^{\prime}(t)$ we can say that all the reliability characteristics

$$
\begin{aligned}
& R(t)=\text { reliability function, } \\
& F(t)=\text { failure distribution function, } \\
& f(t)=\text { failure density function, } \\
& \lambda(t)=\text { failure rate function, }
\end{aligned}
$$

are known if one of them is known. It is therefore often a matter of convenience, of avai1able statistical data or other information, which one of them is first determined, or which one is calculated at all. Very often it occurs that only $R(t)$ is needed and this only for a special value of the time $t$. It also occurs very often that not enough information is given to determine any of the mentioned functions, but that we can only say something about the average lifetime of the system. In this case we say that we determine the mean time to failure (MTTF) of an unrepairable system. It is defined as the expected value of the lifetime $\mathrm{T}$ of the system and is given by

$$
\text { MTTF }=\int_{0}^{\infty} t d F(t)=\int_{0}^{\infty} t f(t) d t .
$$

(Throughout the whole report we shall always assume that MTTF is finite.) Using partial integration it can easily be shown that

$$
\text { MTTF }=\int_{0}^{\infty} R(t) d t,
$$

if, for instance, $\lambda(t) \geq \lambda>0$ for all $t \geq 0$.

Having defined the five most important reliability characteristics, $R(t), F(t), f(t)$, $\lambda(t)$, and MTTF for unrepairable systems we shall discuss typical failure distributions which are often used in practice to find analytical expressions for the reliability characteristics of existing components and systems.

\section{TYPICAL FAILURE DISTRIBUTIONS}

There exist typical failure distributions which depend on unknown parameters. These parameters have to be determined to adjust the distribution to the particular system under 
consideration. The determination of these parameters is a matter of statistical estimation which we do not discuss here. A few comments, however, on how to do that are given in Section 6 of the Appendix. The most important failure distributions used in practice are given in Table 1. The simplest and at the same time mostly applied failure distribution is the exponential failure distribution.

Table 1

Typical failure distributions

\begin{tabular}{|l|c|c|}
\cline { 2 - 4 } \multicolumn{1}{c|}{} & Failure density $f(t)$ & $\lambda(t)$ \\
\hline Exponential & $\lambda \mathrm{e}^{-\lambda t} ; \quad\left[R(t)=\mathrm{e}^{-\lambda t}\right]$ & $\lambda$ \\
\hline Gamma & $\lambda(\lambda t)^{\alpha-1} \mathrm{e}^{-\lambda t} / \Gamma(\alpha) ; \quad \alpha, \lambda>0$, & - \\
\hline Weibul1 & $\lambda \alpha \mathrm{t}^{\alpha-1} \mathrm{e}^{-\lambda t^{\alpha}} ; \quad \alpha, \lambda>0, \quad\left[R(t)=\mathrm{e}^{-\lambda t^{\alpha}}\right]$ & $\lambda \alpha t^{\alpha-1}$ \\
\hline $\begin{array}{l}\text { Truncated } \\
\text { normal }\end{array}$ & $\exp \left[-\frac{(t-\mu)^{2}}{2 \sigma^{2}}\right] /(a \sigma \sqrt{2 \pi}) ; \quad \begin{array}{l}\sigma>0,-\infty<\mu<+\infty, \\
\text { a normalizing constant }\end{array}$ & - \\
\hline
\end{tabular}

This may be motivated by several facts. A formal but very important reason is its simplicity. In addition, it is the only failure distribution having constant failure rate $\lambda$ which indicates that this failure law can be taken to describe the useful lifetime of components and systems (period II in Fig. 2). Another reason for the practical importance of the exponential law is the following fact. Suppose we have a system consisting of many components. In addition, we assume that

a) the lifetimes of the components are independent;

b) every component failure causes a system failure;

c) failures are detected rather fast and failed components are replaced immediately when detected.

Under these conditions, it can be shown that the failure law of such a system is approximately an exponential law if the system is in operation already for a long time and the number of components is very high ${ }^{6}$ ).

For the exponential law there exists a simple relation between the failure rate $\lambda$ and the mean time to failure MTTF. Let $\mathrm{T}$ be the lifetime of a system having exponential distribution, we easily find that

$$
\operatorname{MTTF}=\int_{0}^{\infty} R(t) d t=\int_{0}^{\infty} e^{-\lambda t} d t=\frac{1}{\lambda} .
$$

There is another interesting property of the exponential failure law. It roughly says that a system having exponential failure distribution does not age. To show that, we consider the conditional probability that $\mathrm{T}>\mathrm{x}+t$ under the condition $\mathrm{T}>t$. In other words, we want to find the reliability of such a system for the time interval of length $x$, supposing 
that the system already operated during the time interval $[0, t]$. From the definition of conditional probability (see Section 3 of the Appendix) we find

$$
P(T>x+t \mid T>t)=\frac{P(\{T>x+t\} \cap\{T>t\})}{P(T>t)}=\frac{P(T>x+t)}{P(T>t)}
$$

or, using formula (I.1) we have

$$
P(T>x+t \mid T>t)=\frac{R(x+t)}{R(t)}
$$

This formula is valid for any failure law of the lifetime $T$. Suppose $T$ has an exponential failure distribution with failure rate $\lambda$. From Table 1 we know that $R(t)=\exp (-\lambda t)$ and from Eq. (I.8) we find

$$
P(T>x+t \mid T>t)=\frac{e^{-\lambda(x+t)}}{e^{-\lambda t}}=e^{-\lambda x}=R(x)
$$

Therefore, formula (I.9) proves the statement that a system with exponential failure law does not age. Naturally, this 1 aw is an idealized model for real systems. It is clear that it can only be used to represent the failure law of components and systems for a certain period of their lifetimes. In practice, for short time missions engineers assume in many cases an exponential law for the lifetimes of components and systems, as we shall see later.

Having a quick look at all the other distributions in Table 1, we observe that all of them are dependent on two unknown parameters which have to be determined in one way or another. We can easily see from the last column in Table 1 that the Weibull distribution has a decreasing failure rate if $\alpha<1$ and an increasing or constant failure rate if $\alpha \geq 1$.
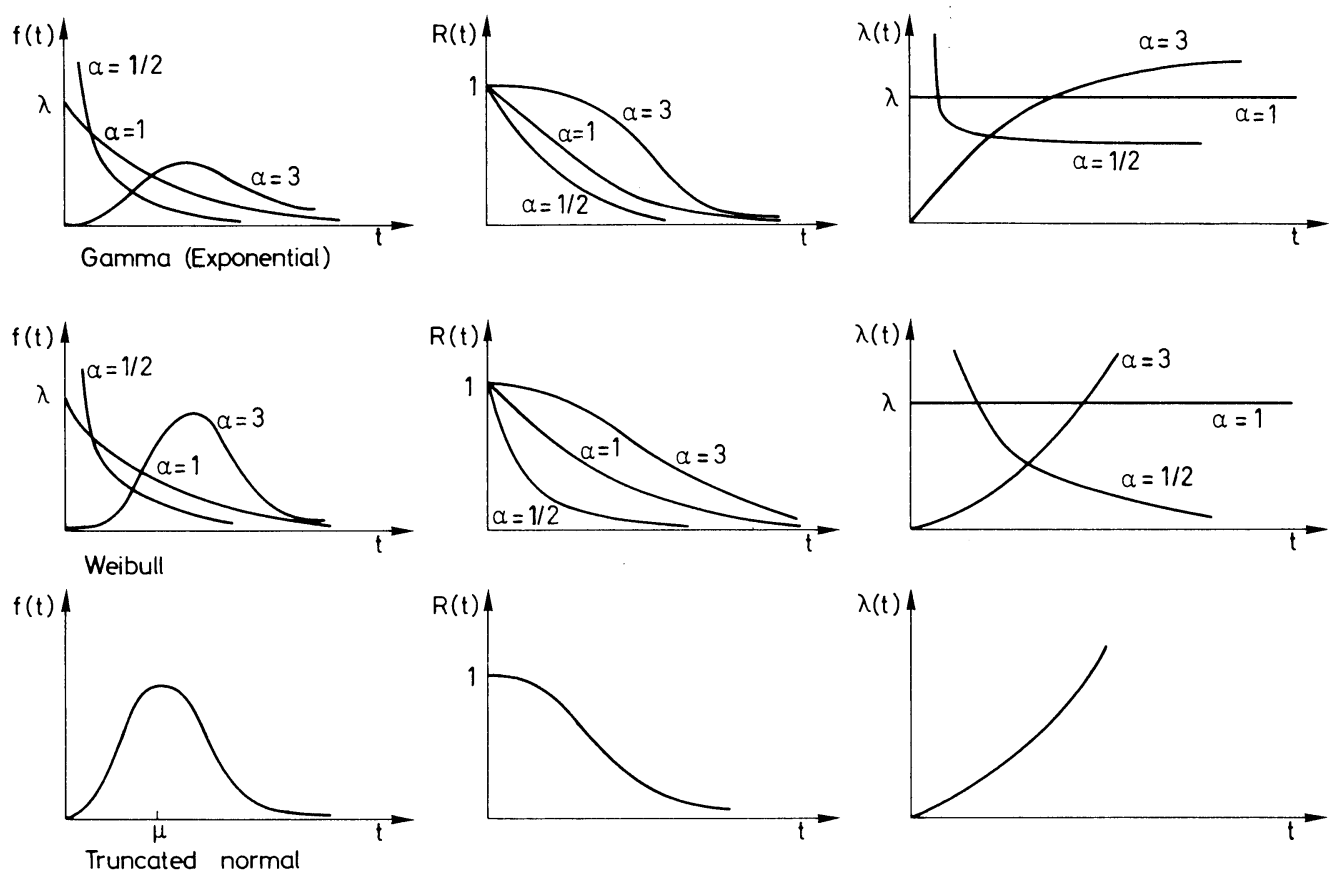

Fig. 3 Typical failure distributions 
The same can be shown for the gamma distribution, but that is more difficult to prove. (The analytical expressions for $\lambda(t)$ of the gamma and the truncated normal distributions are too complicated and therefore not given in Table 1.) This implies that the gamma and the Weibull distributions may be used to describe the failure distributions of systems in all the three different periods given in Fig. 2 by changing the parameter $\alpha$. The truncated normal distribution has always an increasing failure rate and is therefore only suitable for the wear out period. Figure 3 shows examples of $f(t), R(t)$, and $\lambda(t)$ for the different failure laws defined in Table 1.

We mention again that it is basically a statistical problem as to which particular distribution has to be taken in a particular case and how to determine the unknown parameters. The reader may find these problems covered, for instance, in Chapouille and de Pazzis $^{7}$.

\section{IFR FAILURE DISTRIBUTIONS}

For many cases it may neither be possible nor necessary to know the exact failure distribution of a system. In such a case the knowledge that the considered system has an IFR failure distribution may be a big advantage as we shall see soon. We therefore suppose for the moment that the considered failure distribution has increasing failure rate (IFR property). The following theorem can then be proved (see Ref. 6).

\section{Theorem 1}

If $\mathrm{F}(\mathrm{t})$ is IFR with $\mu=\operatorname{MTTF}<\infty$, then

$$
\begin{aligned}
\cdot \exp \left[-\frac{1}{\mu} t\right] & \leq R(t) \leq 1 \quad \text { for } 0 \leq t<\mu, \\
0 & \leq R(t) \leq \exp [-\omega t] \quad \text { for } t \geq \mu
\end{aligned}
$$

where $\omega=\omega(t)$ is the root of the equation

$$
1-\omega \mu=\exp [-\omega t] \text {. }
$$

There exist even more general theorems of this type which can be found in Ref. 6 .

In Theorem 1 we only have to know that $F(t)$ is IFR and what the value of the mean time to failure MTTF is, and the inequalities (I.10) and (I.11) yield us upper and lower bounds for the corresponding reliability function $R(t)$. Figure 4 shows the bounds for $R(t)$ if MTTF $=1$. The lower bound over the interval $0 \leq t<1$ is the exponential function $e^{-t}$. Table 2 shows the upper bound for $1 \leq t \leq 3$.

There exist statistical tests to find out from failure data whether, in a practical case, a considered system has an IFR failure distribution. Such a test is, for instance, described in Ref. 6. The problem of finding an approximate value of MTTF from failure data is an estimation problem for unknown parameters, which is treated, for example, in Ref. 7 . 


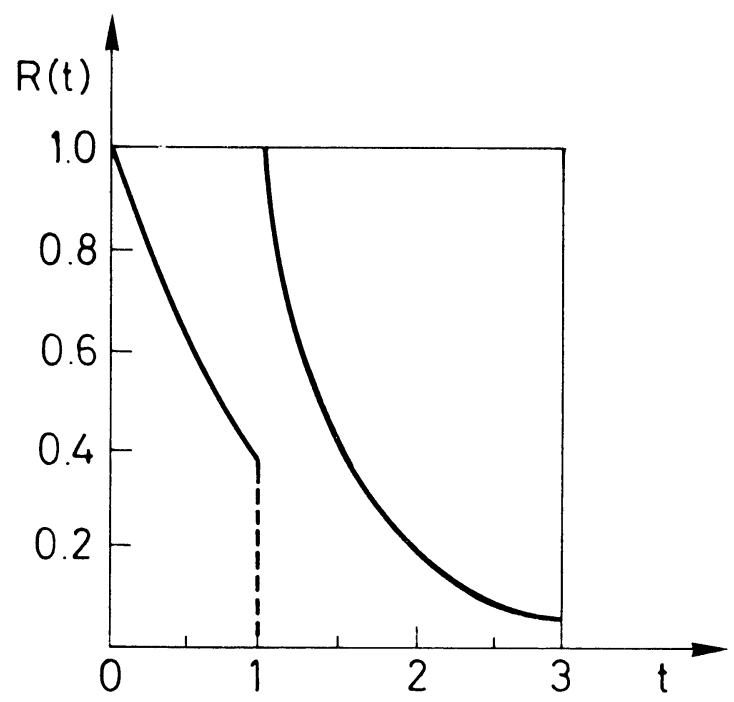

Fig. 4 Upper and lower bounds for $R(t)$ of an IFR distribution (MTTF $=1$ )

Table 2

Upper bound for $R(t)$ of an IFR distribution (MTTF $=\mu=1$ )

\begin{tabular}{|c|c|c|c|}
\hline$t$ & $\begin{array}{c}\text { Upper } \\
\text { bound }\end{array}$ & $t$ & $\begin{array}{c}\text { Upper } \\
\text { bound }\end{array}$ \\
\hline 1.0 & 1.000 & & \\
1.1 & 0.824 & 2.1 & 0.178 \\
1.2 & 0.686 & 2.2 & 0.156 \\
1.3 & 0.577 & 2.3 & 0.138 \\
1.4 & 0.489 & 2.4 & 0.121 \\
1.5 & 0.417 & 2.5 & 0.107 \\
1.6 & 0.358 & 2.6 & 0.095 \\
1.7 & 0.309 & 2.7 & 0.084 \\
1.8 & 0.268 & 2.8 & 0.075 \\
1.9 & 0.233 & 2.9 & 0.067 \\
2.0 & 0.203 & 3.0 & 0.060 \\
\hline
\end{tabular}

\section{SERIES CONNECTIONS}

We already mentioned earlier that the reliability behaviour of systems is highly dependent on the logical connection of its components. We basically have two different kinds of connections of components

- series connections,

- parallel connections, 
where systems with the latter connections are said to have redundancies. There is again a subdivision of redundancies into hot redundancies and cold or stand-by redundancies.

In order to have an idea what these different connections mean, we simply consider the block diagrams of three different systems $S_{i}, i=1,2,3$.

The diagram in Fig. 5 indicates that the system $S_{1}$ fails if $C_{1}$ or $C_{2}$, or both $C_{1}$ and $\mathrm{C}_{2}$, fail. The series connection representation in Fig. 5 must not necessarily mean that $\mathrm{C}_{1}$ and $\mathrm{C}_{2}$ are mechanically or electrically in a series connection. It only shows their logical connection or, in other words, the logical dependence of $\mathrm{S}_{1}$ on these two components. The same holds for the diagrams in Figs. 6 and 7. Of course, in real systems logical series

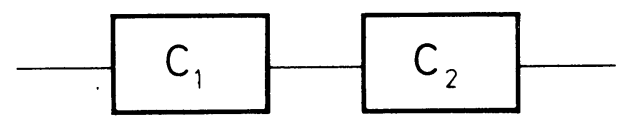

Fig. 5 Series connection $\mathrm{S}_{1}$

connections or parallel connections will mostly coincide with, for instance, mechanical or electrical series or parallel connections. It may, however, happen that, for instance, a mechanical parallel connection must be considered as a series connection in a reliability analysis, as we shall see later in the example of a fuel supply system of an airliner.

The diagram in Fig. 6 indicates firstly that both components are in an operational state and secondly that $\mathrm{S}_{2}$ fails only if $\mathrm{C}_{1}$ and $\mathrm{C}_{2}$ have failed. The expression hot redundancy stresses the fact that both components are in operational state.

The diagram in Fig. 7 indicates that $C_{1}$ is in operational state and $C_{2}$ is stand-by such that whenever $\mathrm{C}_{1}$ fails it can be replaced by $\mathrm{C}_{2}$. (How this is done physically is another question which is not discussed here.) $S_{3}$ like $S_{2}$ fails only if $C_{1}$ and $C_{2}$ have failed.

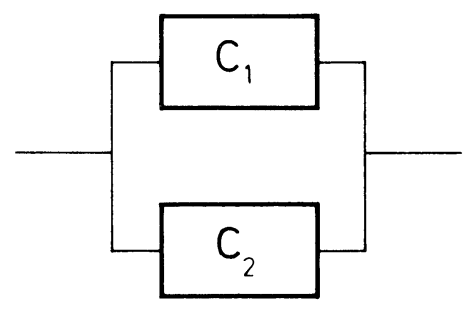

Fig. 6 Parallel connection $\mathrm{S}_{2}$ : hot redundancy

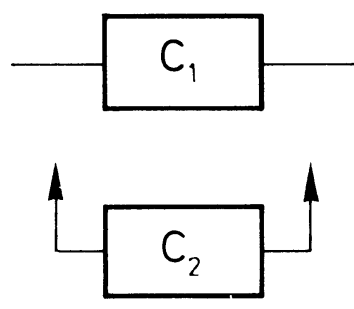

Fig. 7 Parallel connection $\mathrm{S}_{3}$ : cold redundancy

There exist, of course, other connections than series and parallel connections, but we only discuss the latter ones because mostly they occur in practice. Real systems are normally mixtures of series and parallel connections of many components.

It is easy to see that the reliability behaviour of the different connections will be quite different. Let us begin now with the discussion of series connections. In Sections 5 and 6 parallel connections will be covered.

Analogous to the diagram in Fig. 5 we can define what we understand by a series connection of $n$ components. 


\section{Definition 3}

A series connection of $n$ components $C_{1}, \ldots, C_{n}$ is a system consisting of these components and failing when at least one of the $C_{i}$ fails.

We now ask the question: "What is the reliability of a series connection of $\mathrm{n}$ unrepairable components $C_{1}, \ldots, C_{n}$, having independent lifetimes $T_{1}, \ldots, T_{n}$ and given reliability functions $R_{1}(t), \ldots, R_{n}(t)$ ?". The following theorem answers the question.

\section{Theorem 2}

A system $\mathrm{S}$ consisting of $\mathrm{n}$ unrepairable components $\mathrm{C}_{1}, \ldots, \mathrm{C}_{\mathrm{n}}$ in series connection with independent lifetimes $\mathrm{T}_{1}, \ldots, \mathrm{T}_{\mathrm{n}}$ of the components has the reliability function

$$
R(t)=\prod_{i=1}^{n} R_{i}(t)
$$

where $R_{i}(t)$ is the reliability function of $C_{i}$.

Proof. Let T be the lifetime of the system S. We then have

$$
R(t)=P(T>t)=P\left(T_{1}>t, \ldots, T_{n}>t\right) .
$$

Since the $T_{i}$ are independent, it follows that

$$
R(t)=\prod_{i=1}^{n} P\left(T_{i}>t\right)=\prod_{i=1}^{n} R_{i}(t)
$$

which proves Theorem 2 .

From the proof we can see that the independence of the lifetimes of the components is very important for the result of Theorem 2. In many practical situations we may assume that the lifetimes of the components of a system are practically independent. But there are systems where this is not true. For instance, in each system where the failure of one component puts higher pressure on the other components. Such situations need special treatment; they cannot be handled in a general way.

From Theorem 2 we immediately have the following result for the failure $\lambda(t)$ of the series connection $\mathrm{S}$.

\section{Theorem 3}

Under the conditions of Theorem 2, the failure rate function $\lambda(t)$ of the series connection $\mathrm{S}$ is given by

$$
\lambda(t)=\sum_{i=1}^{n} \lambda_{i}(t),
$$

where $\lambda_{i}(t)$ is the failure rate function of $C_{i}$.

In particular, if the components have constant failure rates $\lambda_{i}, i=1, \ldots, n$, the system $\mathrm{S}$ has constant failure rate

$$
\lambda=\sum_{i=1}^{n} \lambda_{i},
$$


Theorem 3 immediately follows from Theorem 2 and formula (I.4). It shows that even in the case where each component has a small failure rate $\lambda_{i}(t)$, the series connection $S$ can have a large failure rate if $\mathrm{n}$ is large.

As an example for the reliability of a series connection, we take $n$ components with the same constant failure rates $\lambda_{i}=1$ for $i=1, \ldots, n$. Then, the reliability function $R(t)=R(t ; n)$ of the corresponding series connection is given by

$$
R(t ; n)=e^{-n t}
$$

Figure 8 shows $R(t ; n)$ for different $n$.

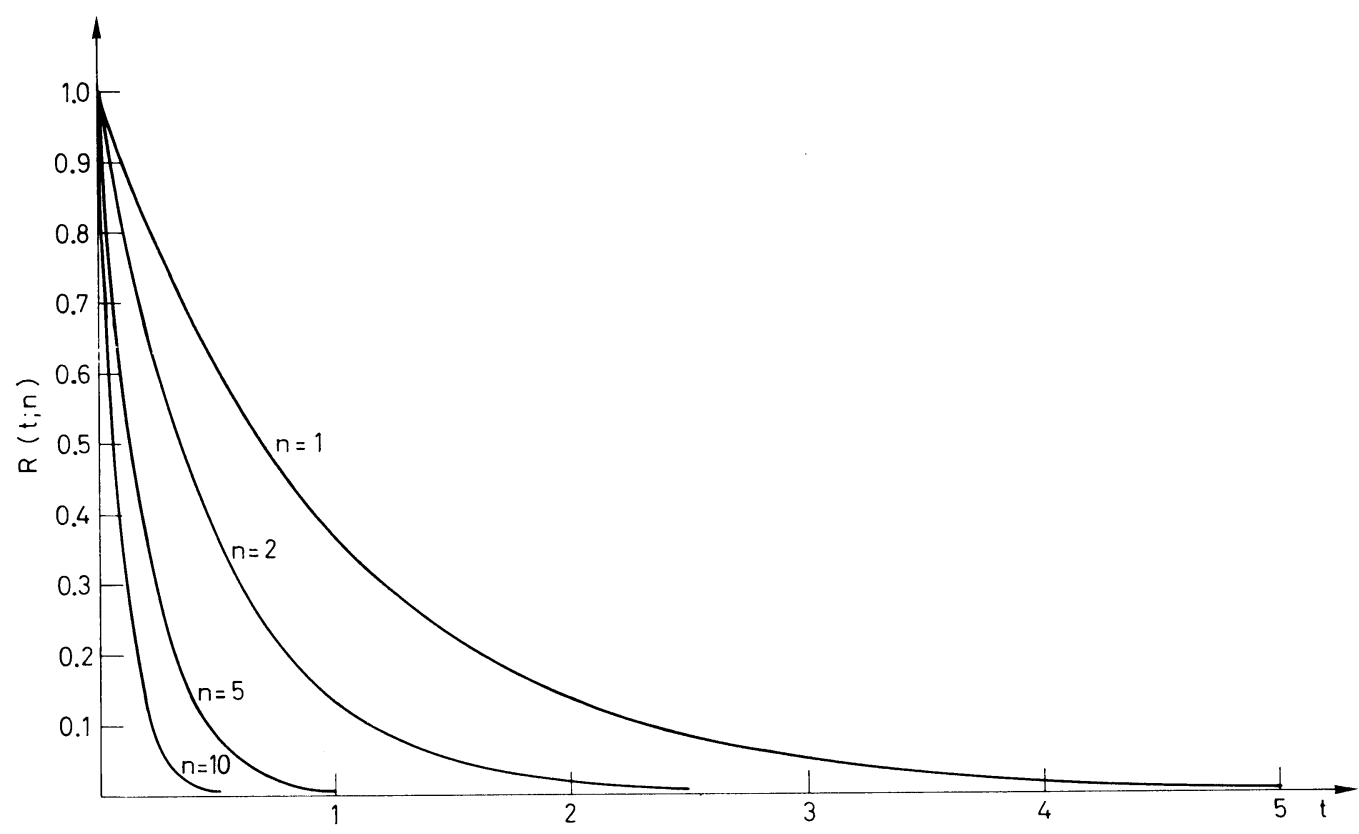

Fig. 8 Reliability of a series connection of $n$ equal components with failure rates $\lambda_{i}=1$

We are also interested in the mean time to failure MTTF of our series connection S. In the general case, it has to be calculated with the aid of Eqs. (I.13) and (I.6). However, in the case where the failure rates of all components are constant, we obtain from Eqs. (I.7) and (I.15):

$$
\operatorname{MTTF}=\frac{1}{\sum_{i=1}^{n} \lambda_{i}} .
$$

And, if $\lambda_{i}=\lambda=1 /$ MTTF $_{\text {comp }}$, we have

$$
\operatorname{MTTF}=\frac{1}{n} \operatorname{MTTF}_{\text {comp }}
$$




\section{PARALLEL CONNECTIONS (HOT REDUNDANCIES)}

We have seen that series connections can become very unreliable if they consist of a large number of components. It is, however, impossible to design real systems without series connections. We therefore have to do something to prevent systems from becoming too unreliable. One possibility is to take more reliable components. But in most of the practical cases there is only one kind for each of the different components available and we have to live with that situation. We must therefore use the possibility of designing systems with redundant components. For, we shall show that this way it is possible to design, at least theoretically, systems which have any desired degree of reliability, using components which may not be very reliable. However, in reality, we will very quickly run into problems with respect to cost, weight, volume, etc., of a system if we want to make the system extremely reliable by using redundant components. Problems of this type are discussed in Chapter III, where optimization aspects are taken into account. .

Let us now first define what, in general, we understand by a parallel connection. We already know from Section 4 that we distinguish between two different types of parallel connections: hot redundancies and cold or stand-by redundancies. For shortness we shall refer to these two connections as hot parallel and cold parallel connections. Generally, a hot parallel connection is defined as follows.

\section{Definition 4}

A hot parallel connection of $n$ components $C_{1}, \ldots, C_{n}$ is a system consisting of these components in operating state and failing only if all components have failed.

A block diagram of such a system for $\mathrm{n}=2$ is given in Fig. 6 .

Asking the question as to what the reliability of a hot parallel connection of $n$ unrepairable components with independent lifetimes is, we find the answer in:

\section{Theorem 4}

A system $S$ consisting of $n$ unrepairable components $C_{1}, \ldots, C_{n}$ in hot parallel connection with independent lifetimes $\mathrm{T}_{1}, \ldots, \mathrm{T}_{\mathrm{n}}$ of the components has the reliability function

$$
R(t)=1-\prod_{i=1}^{n}\left[1-R_{i}(t)\right] \text {, }
$$

where $R_{i}(t)$ is the reliability function of $C_{i}$.

Proof. Let $\mathrm{T}$ be the lifetime of the system $\mathrm{S}$. We then have

$$
R(t)=1-P(T \leq t)=1-P\left(T_{1} \leq t, \ldots, T_{n} \leq t\right)
$$

This formula represents the fact that the system $\mathrm{S}$ fails if, and only if, all the components have failed. Since the lifetimes of the components are independent, we have from Eq. (I.20):

$$
R(t)=1-\prod_{i=1}^{n} P\left(T_{i} \leq t\right)=1-\prod_{i=1}^{n}\left[1-P\left(T_{i}>t\right)\right],
$$

which is identical with Eq. (I.19). Thus, Theorem 4 is proved. 
From the formula (I.19) we can see that the expression for $R(t)$ of a hot parallel connection is only slightly more complicated than the corresponding one for a series connection (Theorem 2). But this already leads to the fact that there exists no corresponding formula for the failure rate function $\lambda(t)$ of a hot parallel connection as we have it in the formula (I.14) for a series connection. In other words, here we have to use the formula (I.19) and put it in the defining equation (I.2) of the failure rate function to find $\lambda(t)$ for a hot parallel connection in each special case.

In order to get a feeling of how much redundancy can improve the reliability of a system, we consider the special case where all the $n$ components of a hot parallel connection have the same constant failure rate $\lambda_{0}$. According to Eq. (I.19) we then have

$$
R(t)=R\left(t ; n, \lambda_{0}\right)=1-\left(1-e^{-\lambda_{0} t}\right)^{n},
$$

which shows that $R\left(t ; n, \lambda_{0}\right)$ tends to 1 for all $\lambda_{0}>0, t \geq 0$, and $n \rightarrow \infty$. In Fig. 9 the function $R(t ; n, 1)$ is plotted for different $n$.

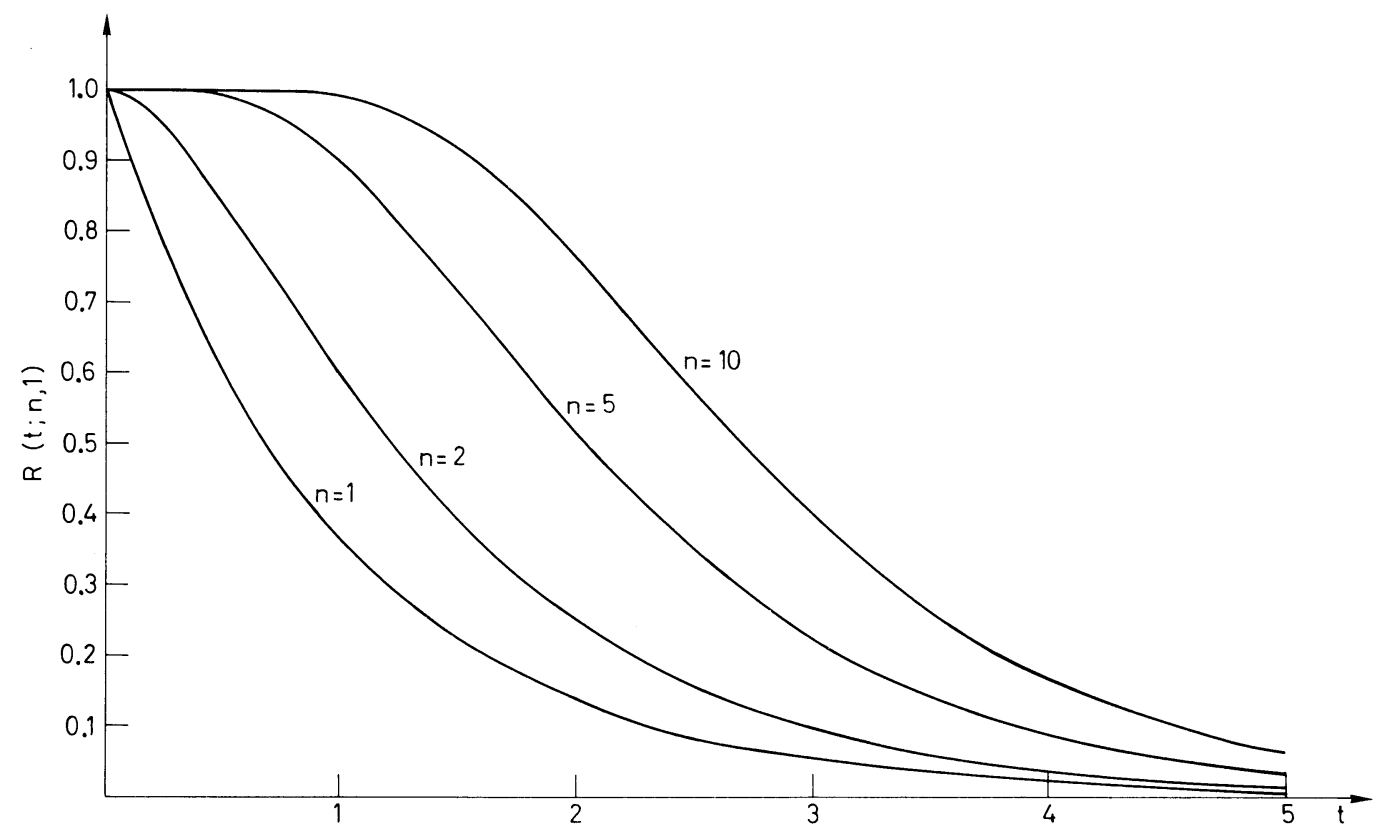

Fig. 9 Reliability of a hot parallel connection of $n$ components with equal failure rate $\lambda_{0}=1$

From Eq. (I.21) we see that a hot parallel connection of $n$ components with equal constant failure rate does not have a constant failure rate itself. Using Eq. (I.2) in the form

$$
\lambda\left(t ; n, \lambda_{0}\right)=-\frac{R^{\prime}\left(t ; n, \lambda_{0}\right)}{R\left(t ; n, \lambda_{0}\right)},
$$

we easily find from Eqs. (I.21) and (I.22) the expression

$$
\lambda\left(t ; n, \lambda_{0}\right)=\frac{n \lambda_{0}}{\sum_{j=0}^{n-1}\left(1-e^{-\lambda_{0} t}\right)^{-j}} .
$$


This formula clearly shows that the failure rate function $\lambda\left(t ; n, \lambda_{0}\right)$ of a hot parallel connection of $\mathrm{n}$ components with equal constant failure rate $\lambda_{0}$ is monotonically increasing (IFR failure distribution). In addition, we see that $\lambda\left(t ; n, \lambda_{0}\right)$ tends to $\lambda_{0}$ for $t \rightarrow \infty$. Figure 10 shows $\lambda(t ; n, 1)$ for different $n$.

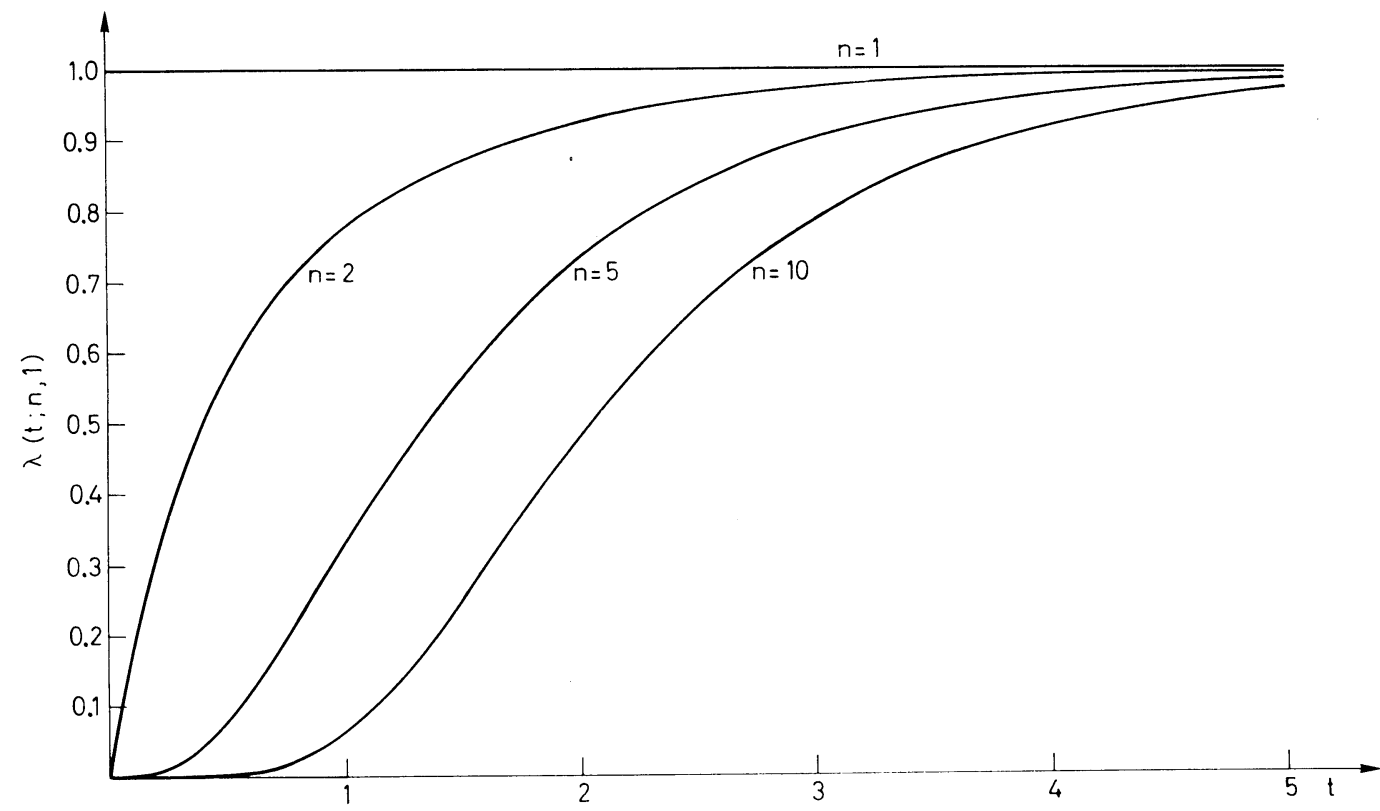

Fig. 10 Failure rate of a hot parallel connection of $n$ components with equal failure rate $\lambda_{0}=1$

The mean time to failure MTTF of a hot parallel connection can generally be calculated by using the formulae (I.19) and (I.6). For our special example we obtain from Eq. (I.21) and (I.6):

$$
\begin{aligned}
\text { MTTF } & =\int_{0}^{\infty}\left[1-\left(1-\mathrm{e}^{-\lambda_{0} t}\right)\right]^{\mathrm{n}} \mathrm{dt} \\
& =\frac{1}{\lambda_{0}} \int_{0}^{1} \frac{1-\mathrm{y}^{\mathrm{n}}}{1-\mathrm{y}} \mathrm{dy}=\frac{1}{\lambda_{0}} \sum_{\mathrm{k}=0}^{\mathrm{n}-1} \int_{0}^{1} y^{\mathrm{k}} \mathrm{dy}
\end{aligned}
$$

or

$$
\operatorname{MTTF}=\left(\sum_{k=1}^{\mathrm{n}} \frac{1}{\mathrm{k}}\right) \operatorname{MTTF}_{\text {comp }},
$$

where the transformation $y=1-e^{-\lambda_{0} t}$ was applied in Eq. (I.24) and the notation $1 / \lambda_{0}=$ MTTF $_{\text {comp }}$ is used in formula (I.25).

Let us compare the mean time to failure MTTF ${ }_{\text {ser }}$ of a series connection of $\mathrm{n}$ components

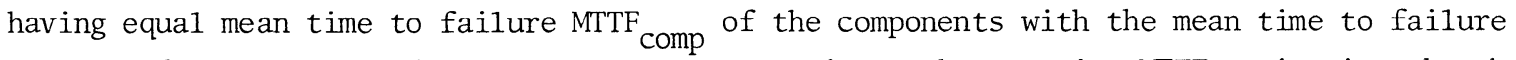
MTTF $_{\text {par }}$ of the corresponding hot parallel connection. The quantity MTTF ${ }_{\text {ser }}$ is given by the

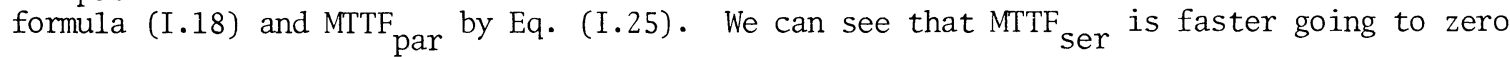
than MTTF ${ }_{\text {par }}$ is going to infinity for $\mathrm{n} \rightarrow \infty$. For instance, a series connection of two equal 
components has half the mean time to failure of a component, but two equal components in hot parallel have only 1.5 times the mean time to failure of a component.

As an example of a combination of series and hot parallel connections we consider a system given by the block diagram in Fig. 11. There we have a hot parallel connection of $n$ equal series connections of two components with equal constant failure rate $\lambda$. We know that each of the series connections of this type has

$$
\operatorname{MTTF}_{\text {ser }}=\frac{1}{2 \lambda}=\frac{1}{2} \operatorname{MTTF}_{\text {comp }} \text {. }
$$

From the formula (I.25) it is easy to see that the system in Fig. 11 has

$$
\operatorname{MTTF}_{\mathrm{S}}=\left(\sum_{\mathrm{k}=1}^{\mathrm{n}} \frac{1}{\mathrm{k}}\right) \mathrm{MTTF}_{\text {ser }}=\left(\frac{1}{2} \sum_{\mathrm{k}=1}^{\mathrm{n}} \frac{1}{\mathrm{k}}\right) \operatorname{MTTF}_{\text {comp }} \cdot
$$

We now ask the question: How to choose $\mathrm{n}$ such that

$$
\mathrm{MTTF}_{\mathrm{S}} \geq \mathrm{MTTF}_{\text {comp }} ?
$$

From Eq. (I.27) we easily see that $\mathrm{n}$ must satisfy the inequality

$$
2 \leq \sum_{\mathrm{k}=1}^{\mathrm{n}} \frac{1}{\mathrm{k}}
$$

which is true if $\mathrm{n} \geq 4$.

In other words, to make the system more or less at least as reliable as one of the components, we need at least four hot redundant series connections of two components.

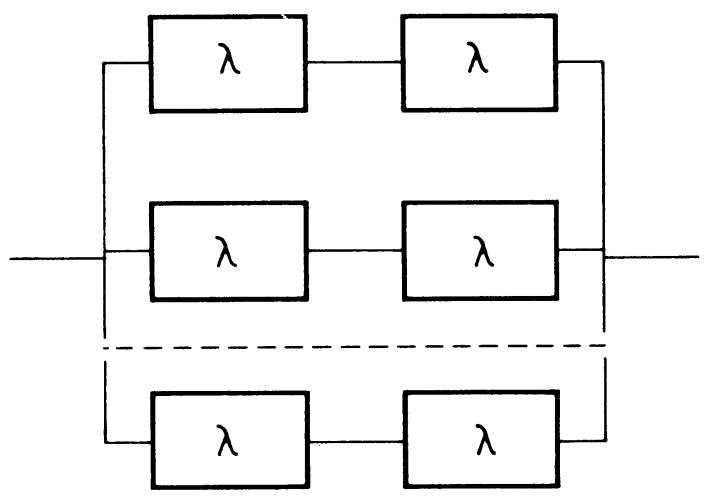

Fig. 11 Hot parallel connection of $n$ equal series connections of two components with equal constant failure rate $\lambda$

\section{PARALLEL CONNECTIONS (COLD REDUNDANCIES)}

In Section 4 we already discussed briefly what we understand by cold redundancies. Their general definition may be given in the following way. 


\section{Definition 5}

The components $\mathrm{C}_{1}, \ldots, \mathrm{C}_{\mathrm{n}}$ are called cold redundant or stand-by components for a system $S_{0}$, if they are not in operating state, but are available to replace components of the system $S_{0}$.

Remark: We assume that cold redundant components do not age as long as they are stand-by.

Among the set of stand-by components we can again distinguish between such components which can replace a failed component immediately by using some kind of a switch, and such stand-by components which need a time-consuming procedure to replace failed components. A single example for the latter case is given by a spare wheel of a car. Stand-by components of this type are discussed in Chapter II, where repairable systems are considered. Here, we shall confine ourselves to the case where we assume having an "ideal switch" for the stand-by components. That means, the "ideal switch" will replace a failed component by a stand-by component immediately after failure and it will do it with certainty, which means that the "ideal switch" has reliability 1. In the literature on reliability also the case where the "ideal switch" is not ideal with respect to reliability is treated (see, for instance, Ref. 2).

It is our goal to find the reliability characteristics of a system $\mathrm{S}$ consisting of a system $S_{0}$ together with $n$ stand-by components $C_{1}, \ldots, C_{n}$ for $S_{0}$. It is too difficult to solve this problem in full generality even with our assumption of an "ideal switch". We sha11 confine ourselves to two special, but important cases.

Case 1. We suppose that the system $S_{0}$ consists of just one component $C_{0}$ for which all the stand-by components are spare parts. In other words, when $\mathrm{C}_{0}$ fails $\mathrm{C}_{1}$ will replace it and so on. Thus, the system $\mathrm{S}$ fails if all components $\mathrm{C}_{0}, \mathrm{C}_{1}, \ldots, \mathrm{C}_{\mathrm{n}}$ have failed.

Case 2. Here, we suppose that the system $\mathrm{S}$ consists of $\mathrm{m}+\mathrm{n}$ equal components with constant failure rate $\lambda$. The first $m$ components form a series connection and represent the system $\mathrm{S}_{0}$, and the remaining $\mathrm{n}$ components are stand-by for $\mathrm{S}_{0}$. If one of the components in $\mathrm{S}_{0}$ fails, it is immediately replaced by a stand-by component. The system $S$ fails after $n+1$ components have failed.

Let us begin with case 1 which is very useful in each situation where $\mathrm{C}_{0}$ and its standby components $C_{1}, \ldots, C_{n}$ are one subsystem of a bigger system. To find the reliability characteristics of the bigger system, we also need those of the system considered in case 1 . Therefore, this case is not as special as it might look at first glance.

We denote the lifetime of $C_{i}$ by $X_{i}$, and $T_{j}$ will denote the lifetime of the system $S_{j}$ consisting of $S_{0}=C_{0}$ plus $C_{1}, \ldots, C_{j}$ as stand-by components for $S_{0}$. With this notation our system $S$ is then equal to $S_{n}$ of which we want to find the reliability characteristics.

Because of the fact that we assumed an "ideal switch", it is immediately seen that

$$
T_{j}=\sum_{i=0}^{j} X_{i}, \quad j=1,2, \ldots, n .
$$

Since $T_{j}$ is the sum of $j+1$ independent random variables -- the $x_{i}$ are supposed to be independent -- we can apply the results of Section 9 of the Appendix to find the distribution function $F_{n}\left(t_{n}\right)$ of the lifetime $T_{n}$ of the system $S_{n}=S$. 
We suppose that we know the distribution functions $G_{i}\left(x_{i}\right)$ of the lifetime $x_{i}$ of the components $C_{i}$ for $i=0,1, \ldots, n$. Taking into account that the functions $G_{i}\left(x_{i}\right)$ and the distribution functions $F_{j}\left(x_{j}\right)$ of the lifetimes $T_{j}$ vanish for negative arguments, the formula (A.49) in the Appendix easily yields the recurrence relation

$$
F_{j}(t)=\int_{0}^{t} G_{j}(t-\tau) d F_{j-1}(\tau)
$$

for $j=1,2, \ldots, n$, where $F_{0}(t)=G_{0}(t)$.

Using formula (I.29) it is now easy to find a recurrence relation for $R_{j}(t)=1-F_{j}(t)$, where $R_{j}(t)$ is the reliability of the system $S_{j}$. The result is given by the formula

$$
R_{j}(t)=R_{j-1}(t)-\int_{0}^{t} P_{j}(t-\tau) d R_{j-1}(\tau)
$$

for $j=1,2, \ldots, n$, where

$$
\begin{aligned}
& R_{0}(t)=P_{0}(t), \\
& P_{j}(t)=1-G_{j}(t), \quad j=0,1,2, \ldots, n .
\end{aligned}
$$

Equation (I.30) allows us to find the reliability $R(t)=R_{n}(t)$ of the system $S=S_{n}$ in an iterative way.

We formulate the result for case 1 in the following theorem.

Theorem 5

A system $S$ consisting of $n+1$ independent unrepairable components $C_{i}$ with reliability functions $P_{i}(t)$ for $i=0,1, \ldots, n$, where $C_{1}, \ldots, C_{n}$ are stand-by components for $\mathrm{C}_{0}$ and where an "ideal switch" is assumed, has the reliability function $R(t)=R_{n}(t)$ which is obtained from the recurrence relation (I.30).

The failure rate function $\lambda(t)$ for the system $S$ in case 1 has to be computed for each particular case by the aid of formula (I.2). However, the mean time to failure MTTF of $S$ is given by

$$
\operatorname{MTTF}=\sum_{i=0}^{\mathrm{n}} \operatorname{MTTF}\left(\mathrm{C}_{\mathrm{i}}\right),
$$

where $\operatorname{MTTF}\left(C_{i}\right)$ is the mean time to failure of the component $C_{i}$. Equation (I.31) immediately follows from the representation of the lifetime of $S=S_{n}$ in Eq. (I.28).

As an application of Theorem 5 we take the special case where the components have constant failure rates $\lambda_{i}, i=0,1, \ldots, n$, but where all $\lambda_{i}$ are different. (Equal $\lambda_{i}$ will be handled later under case 2.) We are then able to solve the recurrence relation (I.30). The result is formulated in the next theorem. 


\section{Theorem 6}

Let $S$ be a system as in Theorem 5 and let the components have constant failure rates $\lambda_{i}>0, i=0,1, \ldots, n$, which are all distinct. We then have for the reliability function of $\mathrm{S}$ :

$$
R(t)=\prod_{j=0}^{n} \lambda_{j} \sum_{i=0}^{n} \frac{e^{-\lambda_{i} t}}{\lambda_{j} \prod_{\substack{k=0 \\ k \neq i}}^{n}\left(\lambda_{k}-\lambda_{i}\right)},
$$

and for the mean time to failure of $S$ :

$$
\text { MTTF }=\sum_{i=0}^{n} \frac{1}{\lambda_{i}} .
$$

The proof of Eq. (I.32) is found by induction using Eq. (I.30). The formula (I.33) is just a special case of the formula (I.31).

Let us now come to case 2, formulated on page 20. The results for that case are given in the next theorem.

\section{Theorem ?}

Let $S$ be a system of $m+n$ equal independent unrepairable components with constant failure rate $\lambda_{0}$. Let $S_{0}$ be the series connection of the first $m$ components and let the remaining $n$ components be stand-by for $S_{0}$. Under the assumption that there is an "ideal switch" for the stand-by components, the reliability function $R\left(t ; n, m, \lambda_{0}\right)$, the failure rate function $\lambda\left(t ; n, m, \lambda_{0}\right)$ and the mean time to failure $\mathrm{MTTF}_{S}$ of $S$ are given by

$$
\begin{aligned}
R\left(t ; n, m, \lambda_{0}\right) & =\sum_{i=0}^{n} \frac{\left(m \lambda_{0} t\right)^{i}}{i !} e^{-m \lambda_{0} t}, \\
\lambda\left(t ; n, m, \lambda_{0}\right) & =\frac{m \lambda_{0}}{\sum_{i=0}^{n} \frac{n !}{i !\left(m \lambda_{0} t\right)^{n-i}}} \\
\operatorname{MTTF}_{S} & =\frac{n+1}{m} \operatorname{MTTF}_{\text {comp }}
\end{aligned}
$$

We do not prove Theorem 7 in detail. To find Eq. (I.34) methods are used norma1ly which are developed in Queuing Theory. The basic ideas of these methods will be discussed in Chapter II. If $m=1$, however, we have case 1 and we can apply Theorem 5 to prove Eq. (I.34).

Having proved Eq. (I.34) it is straightforward to find formula (I.35) using Eqs. (I.2) and (I.34).

Finally, Eq. (I.36) is obtained by evaluating the integral

$$
\mathrm{MTTF}_{S}=\int_{0}^{\infty} R\left(t ; n, m, \lambda_{0}\right) d t
$$

and setting $\lambda_{0}^{-1}=\mathrm{MTTF}_{\text {comp }}$. 
Figure 12 shows $R(t ; 1, n, 1)$ for different $n$ and Fig. 13 shows the corresponding failure rate functions $\lambda(t ; 1, n, 1)$. In each of the figures we have the curves (dashed curves) of the corresponding hot parallel systems and we observe a big difference. In other words, cold redundant components make a system much more reliable than hot redundant components do. This comes from the fact that we assume cold redundancies do not age as long as they are stand-by, which must not always be true.

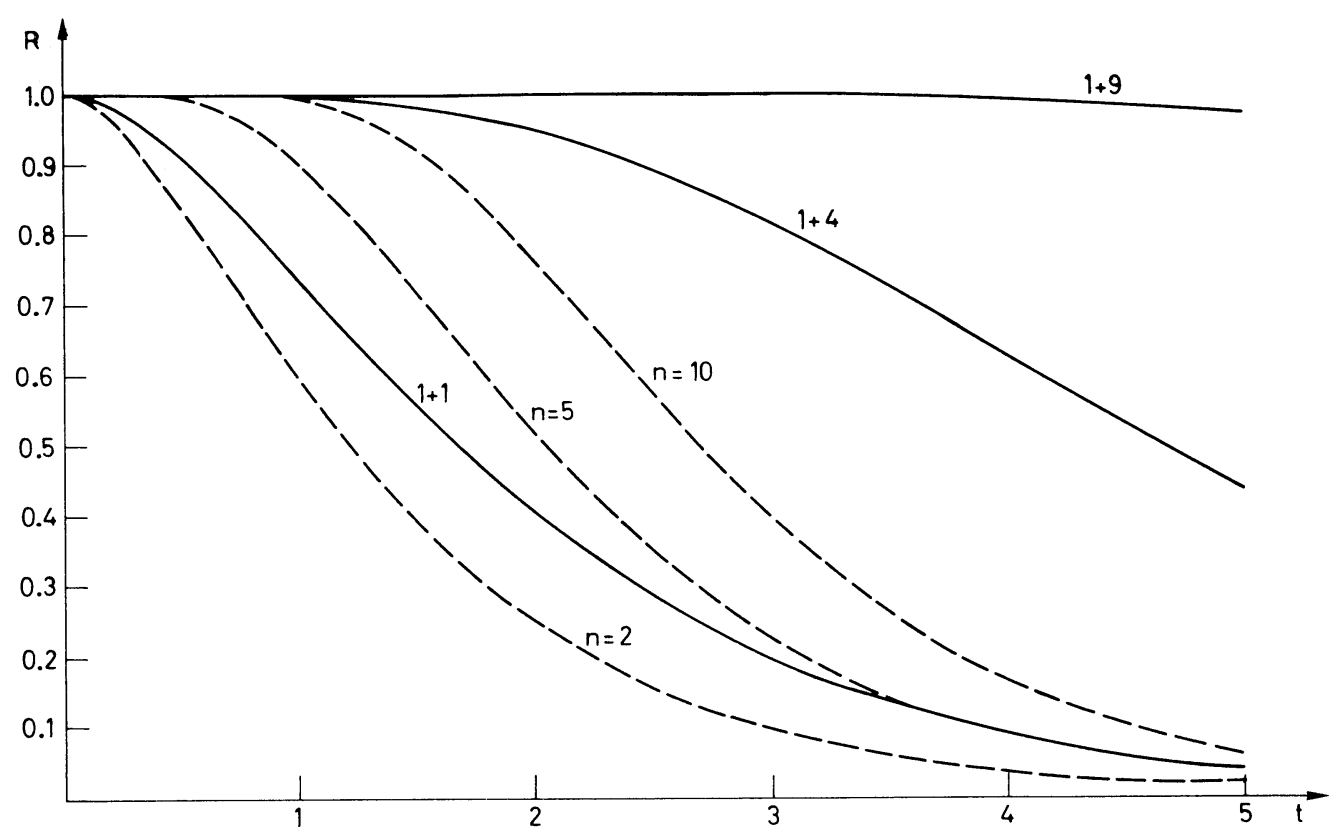

Fig. $12 R(t ; 1, n, 1)$ for different $n$

$R(t ; n, 1)$ for different $n$ (dashed curves)

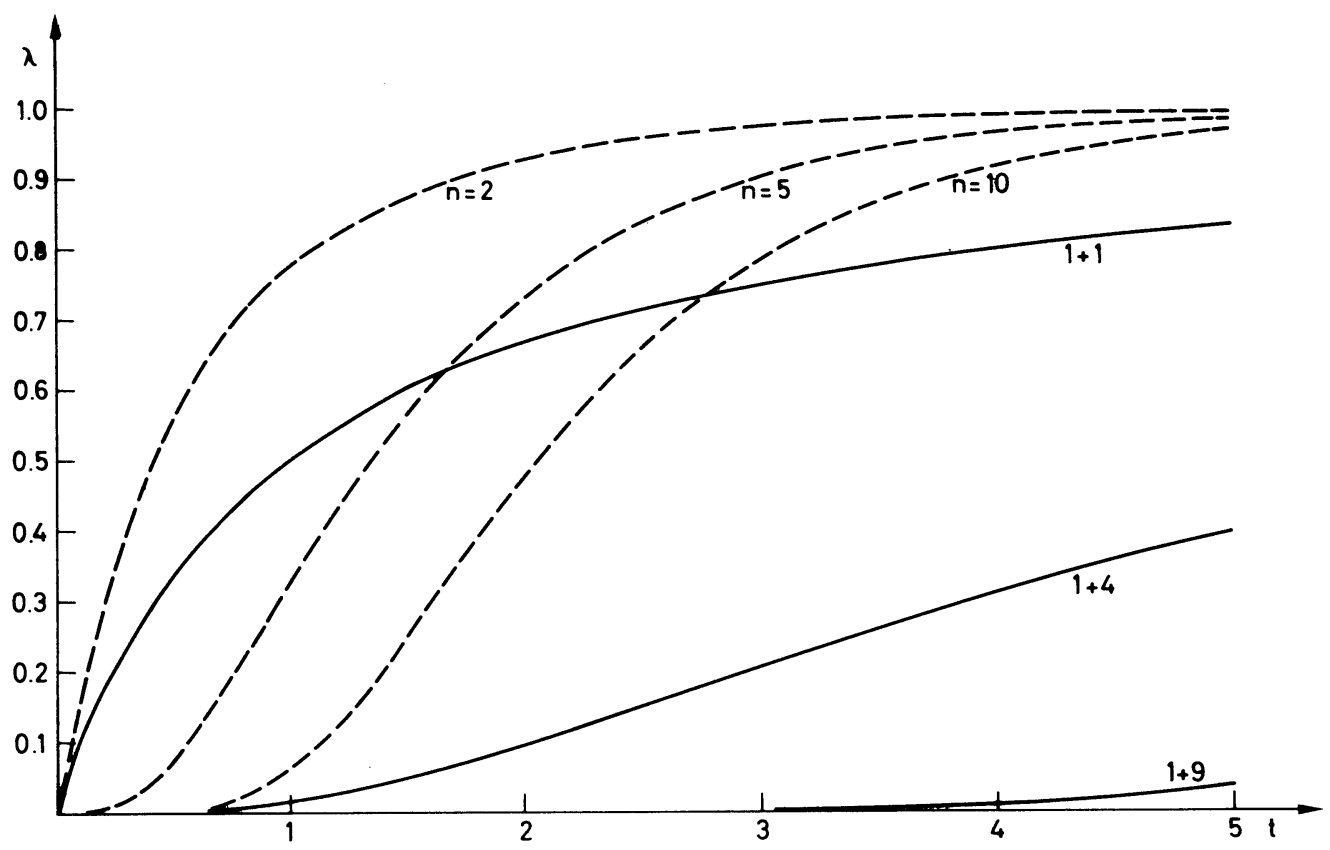

Fig. $13 \lambda(t ; 1, n, 1)$ for different $n$

$\lambda(t ; n, 1)$ for different $n$ (daghed curves) 
Looking at Fig. 13 we observe that $\lambda(t ; 1, n, 1)$ is monotonically increasing. This is generally true for $\lambda\left(t ; n, m, \lambda_{0}\right)$ in Eq. (I.35), as can easily be checked (IFR failure distribution). In addition, one easily finds from Eq. (I.35) that

$$
0 \leq \lambda\left(t ; n, m, \lambda_{0}\right)<m \lambda_{0}
$$

is true for all $t$, where

$$
\lim _{t \rightarrow \infty} \lambda\left(t ; n, m, \lambda_{0}\right)=m \lambda_{0}
$$

We finish this section by considering an example which is analogous to the one we discussed at the end of Section 5. We take in Theorem $7 \mathrm{~m}=2$ and ask the question: How to choose $n$ such that

$$
\mathrm{MTTF}_{\mathrm{S}} \geq \mathrm{MTTF}_{\text {comp }} ?
$$

From Eq. (I.36) we find the answer: If $\mathrm{n} \geq 1$ the inequality (I.39) holds.

Comparing this result now with the result at the end of the previous section, we must say that we need here only $2+1=3$ components, but there we needed at least $4 \times 2=8$ components to satisfy the inequality (I.39) for a system which does the same job but which was designed with hot redundancies.

\section{THE FUEL SUPPLY SYSTEM OF AN AIRL INER}

In this section we discuss an example of a reliability analysis of the design of the fuel supply system of an airliner. The technical details and the figures for the failure rates of the components of the system are taken from a reliability study made at the Dornier Airplane Company ${ }^{*}$ ), Friedrichshafen, Germany, in 1967.

The airliner which we consider has two jet engines, one under each wing. For this airplane we are given two different designs $S_{1}$ and $S_{2}$ of its fuel supply system. We want to calculate the values of two different reliability characteristics for both designs. The first characteristic is the maintenance reliability $\operatorname{MAR}(t)$ defined as the probability that no component of the system fails during the time $[0, t]$. The second characteristic is the operational reliability OPR( $t)$ defined as the probability that at least one of the engines is supplied with fuel during $[0, t]$.

The airliner is a short-range airplane with an average flight time of $t=1.25 \mathrm{~h}$. For this particular flight time $t$ we want to find $\operatorname{MAR}=\operatorname{MAR}(t)$ and $\operatorname{OPR}=O P R(t)$ for $S_{1}$ and $S_{2}$. Besides the calculation of MAR and OPR for both systems, we shall compare these values with the corresponding values of the fuel supply system of the Boeing 737 which is comparable with the designs $S_{1}$ and $S_{2}$.

For our purpose the systems $S_{1}$ and $S_{2}$ are described in the following way. Figure 14 shows a diagram of the system $S_{1}$. The airplane has at each wing an inner tank TI and an outer tank TO. For each tank there is a pump $\mathrm{P}$ with an electrical non-return valve NRV. For each pair of inner and outer tanks at the two wings there is an electrical change-over valve COV. Between the change-over valves $\mathrm{COV}$ and the corresponding engines $\mathrm{E}_{\text {left }}$ and

*) The author wishes to thank his former colleagues at Dornier for the access to these data. 
$E_{\text {right }}$, respectively, we have on both sides an electrical shut-off valve SOV. Finally, all tanks are interconnected by a common pipe which is, between the two inner tanks TI, interrupted by two shut-off valves SOV. Therefore, each engine can get fuel from each tank.

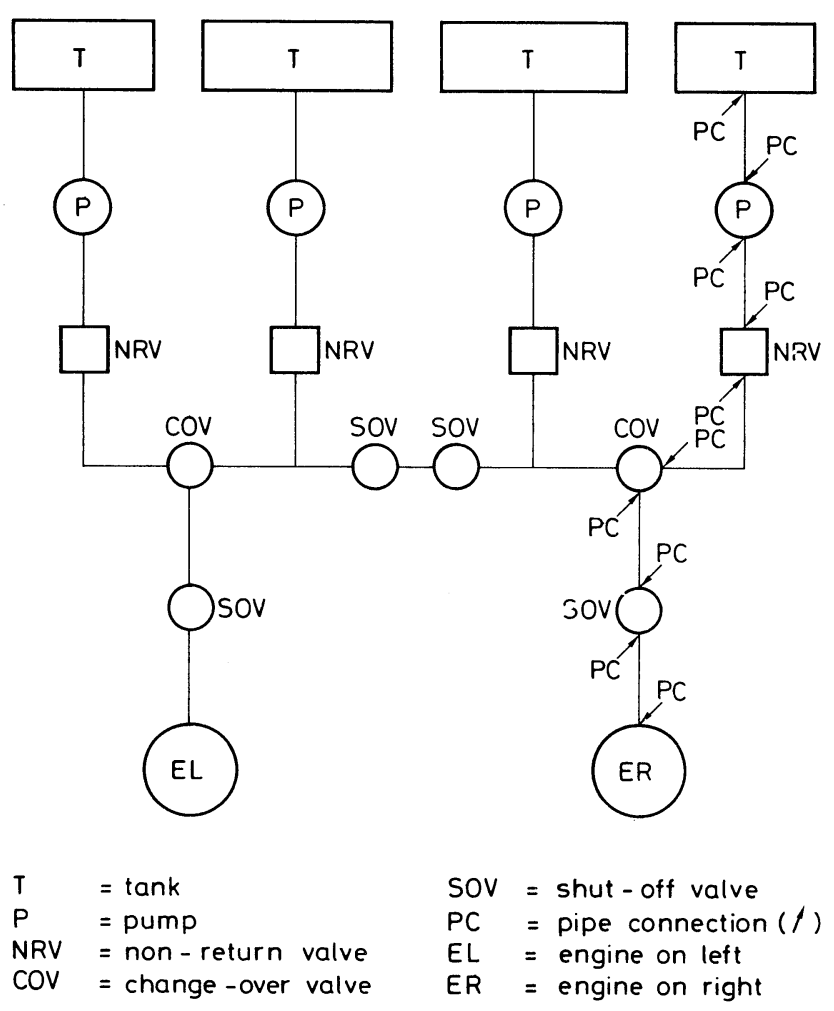

Fig. 14 Design of system $\mathrm{S}_{1}$

Normally the engines take their fuel from either the inner or the outer tanks of the corresponding wings. The choice of the tank is made by the pilot with the corresponding changeover valve COV.

The design $S_{2}$ is very similar to $S_{1}$. The only difference is that for each tank there are two pumps $\mathrm{P}$ plus a non-return valve NOV for each pump in hot parallel connection. That means the system $\mathrm{S}_{2}$ has hot redundancies in the form of the pumps. A diagram of $\mathrm{S}_{2}$ is given in Fig. 15.

We now formulate the assumptions under which we shall make our reliability analysis of $\mathrm{S}_{1}$ and $\mathrm{S}_{2}$.

\section{Assumptions}

a) A11 tanks are full at $t=0$.

b) Each tank contains enough fuel for a flight of $t=1.25 \mathrm{~h}$.

c) A11 components are in their useful lifetimes and have independent lifetimes.

Under these assumptions which have been made in the Dornier study, we only need the failure rates of the components and we are then able to find MAR and OPR of $S_{1}$ and $S_{2}$ using 
Theorems 2, 3, and 4 of this chapter. This is a consequence of assumption (c). The assumptions (a) and (b) will be used when we calculate OPR.

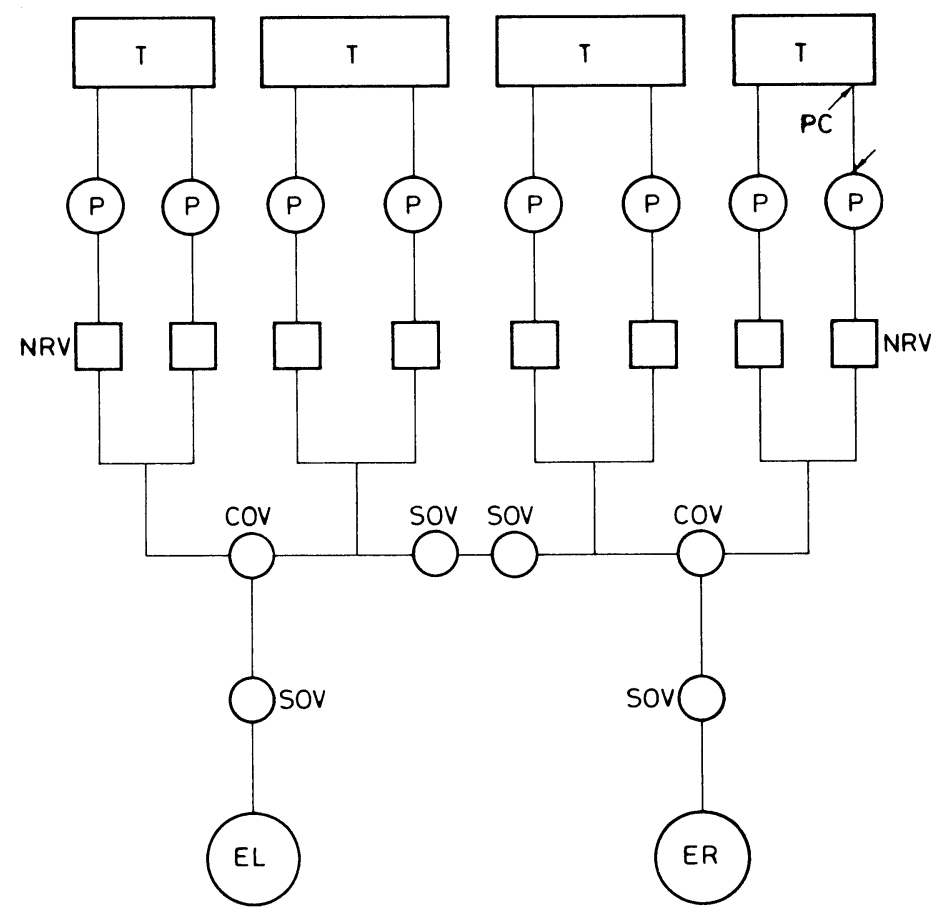

Fig. 15 Design of system $\mathrm{S}_{2}$

Table 3 shows the values of the failure rates $\lambda$ together with the reliabilites $R(1.25)$ and the mean times to failure MTTF of the different components. The failure rates of the tanks can be taken to be zero. The reliability of a pipe is supposed to be essentially determined by its number of connections and the failure rate of these pipe connections. In other words, the failure rate of a pipe is given by the sum of the failure rates of the corresponding pipe connections.

$\underline{\text { Table } 3}$

Numerical values of the characteristics of the components

\begin{tabular}{|l|l|l|c|}
\hline \multicolumn{1}{|c|}{ Component } & $\lambda$ & $\mathrm{R}(1.25)$ & $\begin{array}{c}\text { MTTF } \\
{[\mathrm{h}]}\end{array}$ \\
\hline Tank & 0.0 & 1.0 & $\infty$ \\
\hline Non-return valve & 0.000012 & 0.999985 & 83333 \\
\hline Pump & 0.002173 & 0.997287 & 460 \\
\hline Change-over valve & 0.000283 & 0.999646 & 3534 \\
\hline Shut-off valve & 0.000283 & 0.999646 & 3534 \\
\hline Pipe connection & 0.000002 & 0.999996 & 500000 \\
\hline
\end{tabular}


We first calculate MAR $=\operatorname{MAR}(1.25)$ of both systems. This is particularly easy. Since by definition the maintenance reliability is the probability that at least one of the components fails during the time interval $[0,1.25]$, we must consider the system as a series connection of all components. Since the components all have constant failure rates, the system itself will have a constant failure rate in this case. According to Theorem 3 , the failure rate of the system is the sum of the failure rates of the components. Table 4 shows the sums of the failure rates for $S_{1}$ and $S_{2}$.

\section{Table 4}

Failure rates of $S_{1}$ and $S_{2}$ (maintenance reliability)

\begin{tabular}{|c|c|c|c|}
\hline & $\mathrm{S}_{1}$ & \multicolumn{2}{|r|}{$\mathrm{S}_{2}$} \\
\hline & & $\mathrm{n}_{\mathrm{i}}$ & $\mathrm{n}_{i} \lambda_{i}$ \\
\hline $\mathrm{T}$ & & 4 & 0.0 \\
\hline $\mathrm{P}$ & 692 & 8 & 0.017384 \\
\hline NRV & 048 & 8 & $0.000 \quad 096$ \\
\hline $\mathrm{COV}$ & 132 & 4 & 0.001132 \\
\hline SOV & 132 & 4 & 0.001132 \\
\hline $\mathrm{PC}$ & 076 & 66 & 0.000132 \\
\hline & 080 & $\lambda_{S}$ & .019876 \\
\hline
\end{tabular}

Sincé

we easily find that

$$
\operatorname{MAR}_{i}=\exp \left(-1.25 \lambda_{S_{i}}\right), \quad i=1,2,
$$

$$
\begin{aligned}
& \operatorname{MAR}_{1}=0.986245, \\
& M_{2}=0.975464 .
\end{aligned}
$$

For the corresponding mean times to failure we obtain

$$
\begin{aligned}
& \text { MTTF }_{1}=90.25 \mathrm{~h}, \\
& \text { MTTF }_{2}=50.32 \mathrm{~h} .
\end{aligned}
$$

Dividing MTTF by the average flight time $t=1.25 \mathrm{~h}$, we obtain the average number of missions to repair ENM. We then have

$$
\begin{aligned}
& \mathrm{ENM}_{1}=72.2, \\
& \mathrm{ENM}_{2}=40.3 .
\end{aligned}
$$


It is obvious that the maintenance reliability, the mean time to failure, and the average number of missions to repair are smaller the more components there are in a system, as we can see by comparison of the results for $S_{1}$ and $S_{2}$.

We now come to the determination of the operational reliability OPR of $S_{1}$ and $S_{2}$. We shall do it explicitly for $S_{1}$. For $S_{2}$ the answer will easily follow.

Let us first introduce the following notation.

$E L=$ the event that the engine on the left is supplied with fuel during $[0, t]$.

$E R=$ the event that the engine on the right is supplied with fuel during $[0, t]$. Since the operational reliability OPR was defined as the probability that at least one of the engines is supplied with fuel during $[0, t]$, we have

$$
\mathrm{OPR}=\mathrm{P}(\mathrm{EL} \cup \mathrm{ER})
$$

as is easily seen by interpreting the right-hand side of Eq. (I.40). According to formula (A.4) of the Appendix we have

$$
P(E L \cup E R)=P(E L)+P(E R)-P(E L \cap E R)
$$

The symmetry of the systems $S_{1}$ and $S_{2}$ implies that

$$
P(E R)=P(E L) \text {. }
$$

We therefore obtain

$$
\mathrm{OPR}=2 \mathrm{P}(\mathrm{EL})-\mathrm{P}(\mathrm{EL} \cap \mathrm{ER})
$$

From this equation it follows that we know OPR if $P(E L)$ and P(EL $\cap$ ER) are known.

We first calculate $P(E L)$ for $S_{1}$. From the diagram of $S_{1}$ in Fig. 15 it is easy to deduce the block diagram for the operational reliability of $\mathrm{S}_{1}$ given in Fig. 16 . (Here, the assumptions (a) and (b) have to be taken into account.) To calculate

$\mathrm{P}(\mathrm{EL})=$ probability that the engine on the left is supplied with fuel during $[0, t]$, the reliability block diagram in Fig. 16 has to be changed by cutting away the series connection

$$
\mathrm{PC}-\mathrm{PC}-\mathrm{SOV}-\mathrm{PC}-\mathrm{PC}\left(-\mathrm{E}_{\text {right }}\right) \text {. }
$$

Doing this in Fig. 16 and introducing the following abbreviations

$$
\begin{aligned}
& C_{1}=\text { series: } \quad T-P C-P C-P-P C-P C-N R V-P C, \\
& C_{2}=C_{3}=C_{4}=C_{1}, \\
& C_{5}=\text { series: } P C-S O V-P C-P C-S O V-P C, \\
& C_{6}=\text { series: } P C-P C-S O V-P C-P C, \\
& C_{7}=C_{6},
\end{aligned}
$$




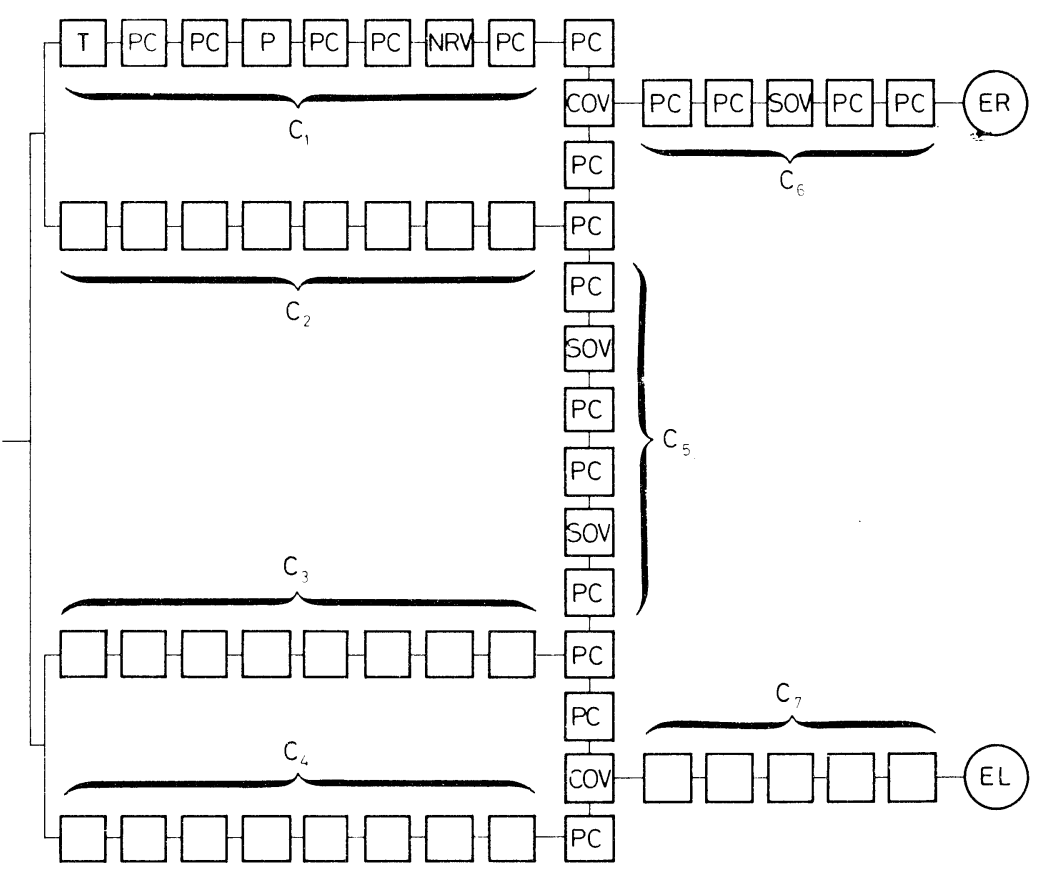

Fig. 16 Reliability block diagram for $\mathrm{S}_{1}$

the corresponding block diagram to calculate $\mathrm{P}(\mathrm{EL})$ can be represented as in Fig. 17 . Again introducing abbreviations we can successively reduce the block diagram for the calculation of $\mathrm{P}(\mathrm{EL})$. This has been done in Figs. 18 to 21 . The abbreviations used are the following:
$\mathrm{A}_{1}=$ series: $\mathrm{C}_{1}-\mathrm{PC}-\mathrm{COV}-\mathrm{PC}$,
$\mathrm{C}_{12}=$ series: $\mathrm{COV}-\mathrm{C}_{7}$,
$\mathrm{C}_{8}=$ hot parallel: $\mathrm{A}_{1}-\mathrm{C}_{2}$,
$A_{2}=$ series: $C_{8}-C_{10}$,
$\mathrm{C}_{9}=$ series: $\mathrm{C}_{4}-\mathrm{PC}$,
$\mathrm{C}_{13}=$ hot paralle1: $\mathrm{A}_{2}-\mathrm{C}_{3}$,
$\mathrm{C}_{10}=$ series: $\mathrm{PC}-\mathrm{C}_{5}$,
$C_{14}=$ series: $C_{13}-C_{11}$,
$\mathrm{C}_{11}=$ series: $\mathrm{PC}-\mathrm{PC}$,
$\mathrm{C}_{15}=$ hot parallel: $\mathrm{C}_{14}-\mathrm{C}_{9}$.

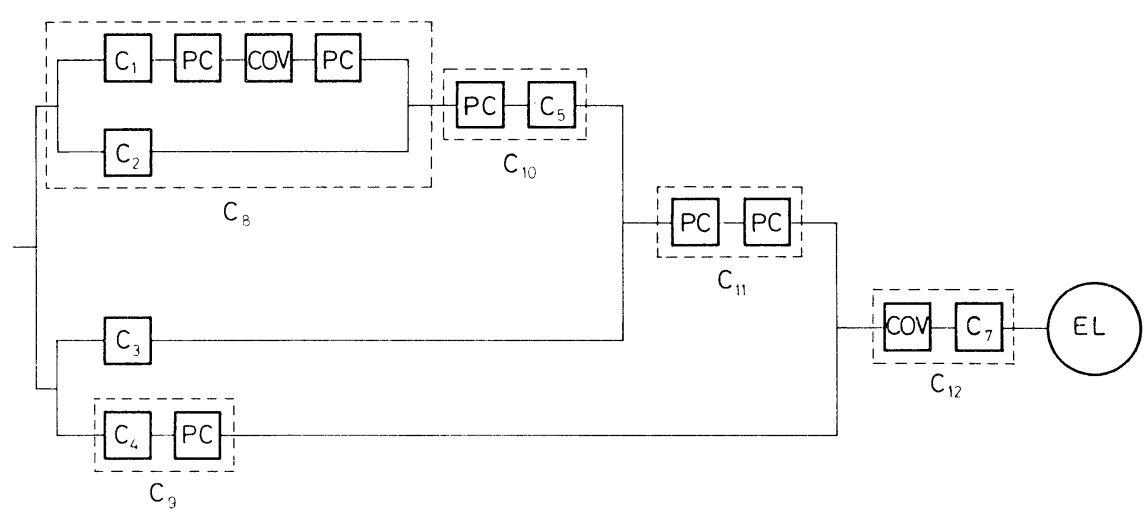

Fig. 17 Reliability diagram for the calculation of P(EL) 


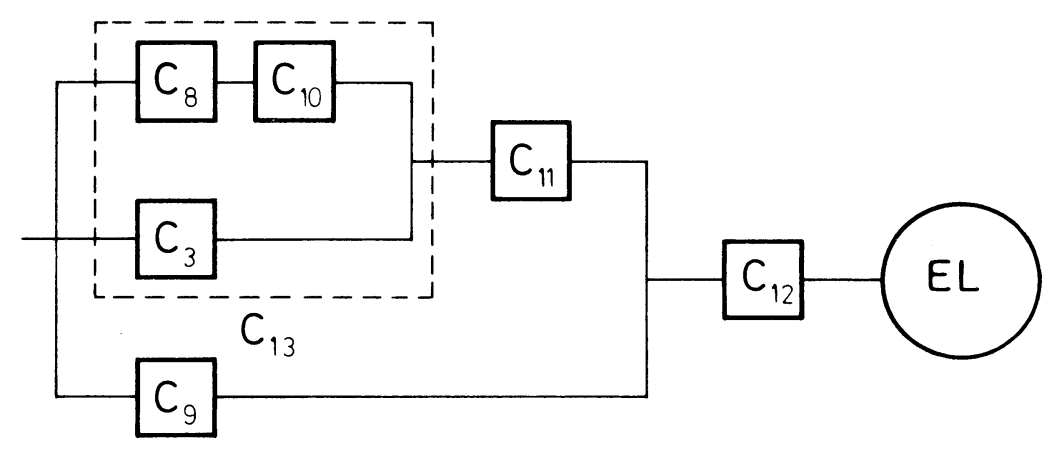

Fig. 18 Reduction of Fig. 17

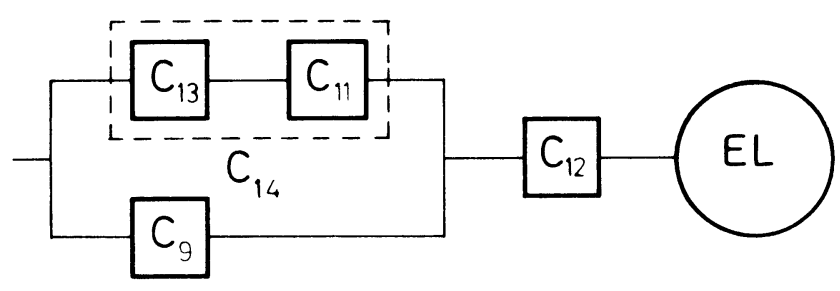

Fig. 19 Reduction of Fig. 18

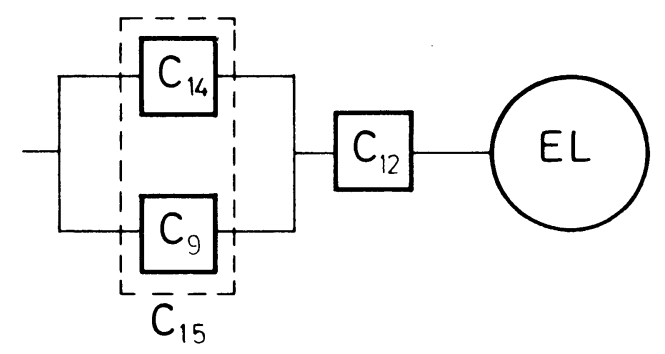

Fig. 20 Reduction of Fig. 19

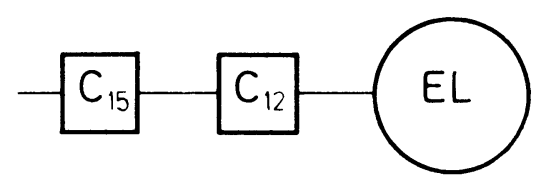

Fig. 21 Reduction of Fig. 20

Looking at the way we defined the subsystems $C_{i}, i=1, \ldots, 15$ and $A_{1}, A_{2}$, we easily find that the event EL will occur if the series

$$
\mathrm{C}_{15}-\mathrm{C}_{12}
$$

in Fig. 21 works. Therefore, if we denote by $R_{C_{i}}, i=1, \ldots, 15$ the reliabilities of the $C_{i}$, it immediately follows from Theorem 2 that

$$
P(E L)=R_{C_{12}} R_{C_{15}}
$$


In order to find $R_{C_{12}}$ and $R_{C_{15}}$ we have to calculate successively the $R_{C_{i}}, i=1, \ldots, 15$ and $\mathrm{R}_{\mathrm{A}_{1}}, \mathrm{R}_{\mathrm{A}_{2}}$. This can, however, be easily done using Theorem 2 for series connections, and Theorem 4 for hot parallel connections. The calculation is straightforward and we give only the results:

$$
\begin{array}{ll}
\mathrm{R}_{\mathrm{C}_{1}}=0.997252096 & \mathrm{R}_{\mathrm{C}_{10}}=0.999272140 \\
\mathrm{R}_{\mathrm{C}_{2}}=\mathrm{R}_{\mathrm{C}_{3}}=\mathrm{R}_{\mathrm{C}_{4}}=\mathrm{R}_{\mathrm{C}_{1}} & \mathrm{R}_{\mathrm{C}_{11}}=0.999992000 \\
\mathrm{R}_{\mathrm{C}_{5}}=0.999276137 & \mathrm{R}_{\mathrm{C}_{12}}=0.999276137 \\
\mathrm{R}_{C_{6}}=0.999630006 & \mathrm{R}_{\mathrm{A}_{2}}=0.999263603 \\
\mathrm{R}_{\mathrm{C}_{7}}=\mathrm{R}_{\mathrm{C}_{6}} & \mathrm{R}_{\mathrm{C}_{13}}=0.999997977 \\
\mathrm{R}_{\mathrm{A}_{1}}=0.996891093 & \mathrm{R}_{\mathrm{C}_{14}}=0.999989977 \\
\mathrm{R}_{\mathrm{C}_{8}}=0.999991457 & \mathrm{R}_{\mathrm{C}_{15}}=0.999999972 . \\
\mathrm{R}_{\mathrm{C}_{9}}=0.997 \text { 248 107 } &
\end{array}
$$

We now have to find $\mathrm{P}(\mathrm{EL} \cap \mathrm{ER})$ for the system $S_{1}$. A rigorous computation of this probability is possible in principle and can be done using basically the formula (A.4) of the Appendix in an iterative way. But it is a rather lengthy and involved calculation. To avoid it we use an approximate calculation. First of $a 11$, we observe that $\mathrm{R}_{\mathrm{C}_{15}}$ can, since we are at the end of the calculations, be set equal to 1 . Therefore,

$$
P(E L) \approx R_{C_{12}}
$$

Since $S_{1}$ is symmetric, the same is true for $P(E R)$. Looking at the definition of $C_{12}$ and $C_{15}$ it is then easily seen that $P(E L \cap E R)$ is approximately the same as the reliability of a series of two independent subsystems of type $C_{12}$. We therefore have

$$
\mathrm{P}(\mathrm{EL} \cap \mathrm{ER}) \approx \mathrm{R}_{\mathrm{C}_{12}}^{2}
$$

Using the formulae (I.43), (I.45), and (I.46), and inserting the value for $\mathrm{R}_{\mathrm{C}_{12}}$ we obtain

$$
\mathrm{OPR}_{1} \approx 0.999999476
$$

Since $C_{15}$ represents mainly the four pumps in hot parallel in the design $S_{1}$, the corresponding subsystem $C_{15}$ in $S_{2}$ which includes eight pumps in hot parallel must be even more reliable. It turns out that here

$$
\mathrm{R}_{\mathrm{C}_{15}}=0.9999999999 .
$$

However, the corresponding subsystems $C_{12}$ in $S_{2}$ are the same as in $S_{1}$. We therefore have

$$
\mathrm{OPR}_{2} \approx \mathrm{OPR}_{1} \text {. }
$$

When we now compare the result in Eq. (I.48) together with the fact that $\mathrm{MAR}_{2}<\mathrm{MAR}_{1}$, we must come to the conclusion that $S_{1}$ is superior to $S_{2}$, at least as far as the considered 
reliability characteristics are concerned. But $S_{2}$ must obviously have advantages over $S_{1}$. To find these out we consider another reliability characteristic. We call it FUR( $t)$ and define it as the probability that fuel can flow from each tank to the interconnection during $[0, t]$.

Why do we consider this reliability characteristic? We said that $t=1.25 \mathrm{~h}$ is the average flight time of our airliner. That means, however, that in many cases the airplane will also fly longer than $1.25 \mathrm{~h}$, which implies that it needs for such a flight normally more fuel than is contained in one tank. We therefore want to make sure that we can get fuel from each tank with high probability. The characteristic FUR( $t$ ) is a measure for that.

Looking at Fig. 16 and the definition of the subsystems $C_{i}$, $i=1, \ldots, 4$, of system $S_{1}$, it is easily seen that FUR for $S_{1}$ is equal to the reliability of the series connection:

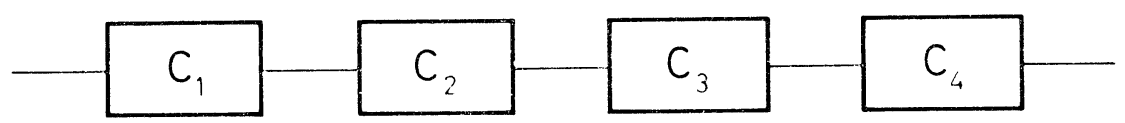

We therefore have

$$
\mathrm{FUR}_{1}=\mathrm{R}_{\mathrm{C}_{1}} \mathrm{R}_{\mathrm{C}_{2}} \mathrm{R}_{\mathrm{C}_{3}} \mathrm{R}_{\mathrm{C}_{4}}=0.989053607
$$

for the system $S_{1}$.

For the system $S_{2}$ we find from Fig. 15 that in this case FUR is given by the reliability of the following connection:

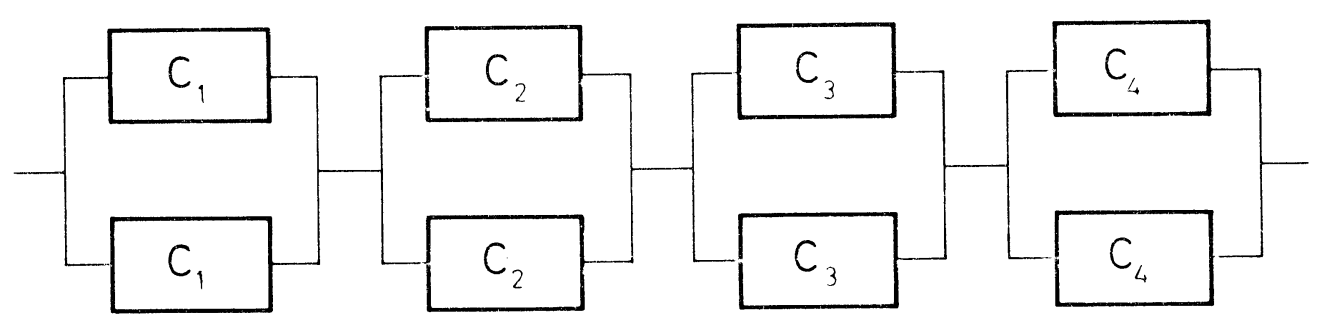

This leads to

$$
\mathrm{FUR}_{2}=\left[1-\left(1-\mathrm{R}_{\mathrm{C}_{1}}\right)^{2}\right]^{4}=0.999969796
$$

where $1-\left(1-R_{C_{1}}\right)^{2}$ is the reliability of the hot parallel connection: $C_{1}-C_{1}$, and where in addition we used the fact that $C_{1}=C_{2}=C_{3}=C_{4}$.

When we now compare $F R_{1}$ and $F R_{2}$ we can see that $S_{2}$ is superior to $S_{1}$ as far as this characteristic is concerned.

What is the conclusion which was drawn in the Dornier reliability study from the results we have mentioned so far? A new design $S_{3}$ was proposed. Its diagram is shown in Fig. 22. There, we have two pumps for each inner tank and one pump for each outer tank. The reason is the following. The inner tanks are the bigger ones and normally only they will be used. The outer tanks will only be used occasionally. Some other changes compared to $\mathrm{S}_{1}$ and $\mathrm{S}_{2}$ are made in $S_{3}$ which are technically possible but which are not further discussed here. 


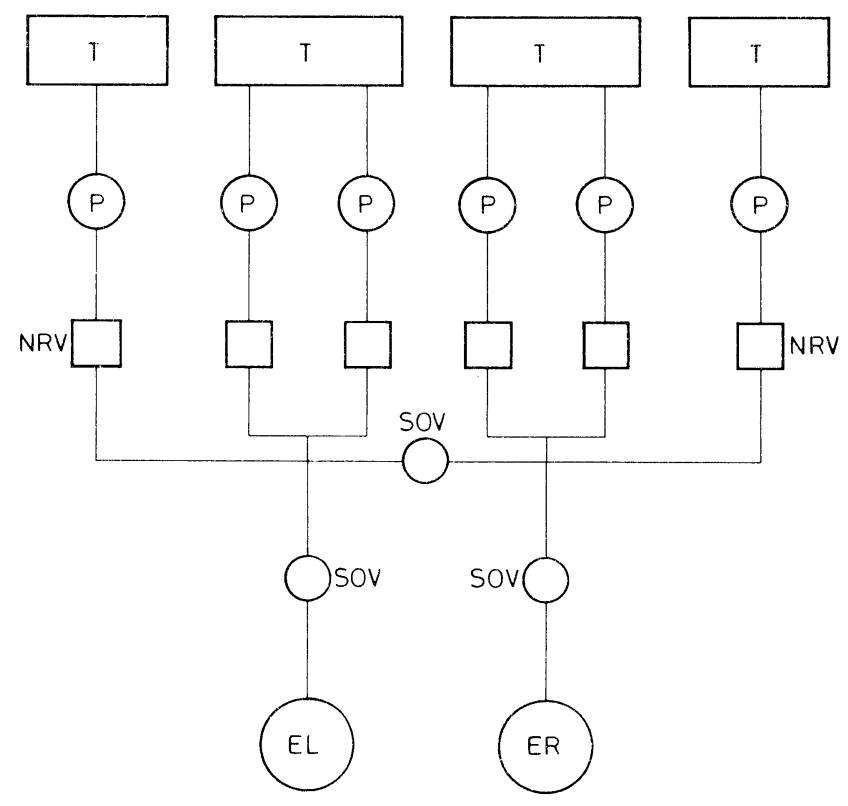

Fig. 22 Diagram of the system $\mathrm{S}_{3}$

The final step we make is a comparison of the designs $S_{1}, S_{2}$, and $S_{3}$ with the fuel supply system $S_{4}$ of the Boeing 737. A diagram of $S_{4}$ is given in Fig. 23. Table 5 shows the figures for the considered reliability characteristics of the different systems $\mathrm{S}_{1}, \ldots, \mathrm{S}_{4}$.

We see that the corresponding values for $\mathrm{S}_{3}$ and $\mathrm{S}_{4}$ are very similar, such that we can say that the new design $S_{3}$ will behave very similarly to an already existing and properly working system.

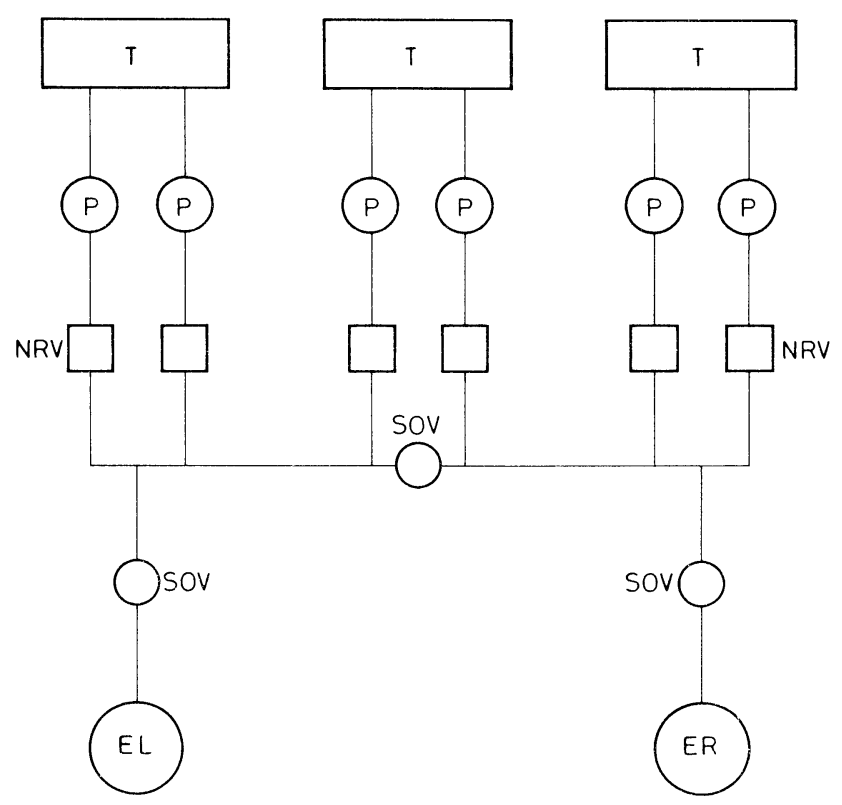

Fig. 23 Diagram of the Boeing 737 system $\mathrm{S}_{4}$ 
Table 5

Comparison of the results

\begin{tabular}{|l|l|l|l|l|}
\hline Characteristic & \multicolumn{1}{|c|}{$\begin{array}{c}\mathrm{S}_{1} \\
\text { pumps }\end{array}$} & $\begin{array}{c}\mathrm{S}_{2} \\
8 \mathrm{pumps}\end{array}$ & $\begin{array}{c}\mathrm{S}_{4} \\
6 \mathrm{pumps}\end{array}$ & $\begin{array}{c}\mathrm{S}_{5} \\
\text { Boeing } 737\end{array}$ \\
\hline MAR & 0.986245 & 0.975464 & 0.982658 & 0.982406 \\
\hline MTTF [h] & 90.25 & 50.32 & 72.2 & 71.0 \\
\hline ENF & 72.2 & 40.3 & 57.7 & 57.0 \\
\hline OPR & 0.9999995 & 0.9999995 & 0.99999993 & 0.99999987 \\
\hline FUR & 0.989054 & 0.999970 & $\begin{array}{c}0.999985] \text { a) } \\
0.994497\end{array}$ & 0.999977 \\
\hline
\end{tabular}

a) The number in parentheses corresponds to the two inner tanks. 


\section{REPAIRABLE SYSTEMS}

\section{DEFINITION OF REL IABILITY CHARACTERISTICS}

The systems and components treated in Chapter I were supposed to be unrepairable, which means that they cannot be repaired after failure to perform their particular tasks even if they can be repaired in principle. In this chapter we will discuss repairable systems. We say that a system is repairable if, after failure, it can be restored by either repairing failed (repairable) components or by replacing these components by new ones. In other words, a repairable system is a system which can be renewed to continue to perform its tasks. It may even occur that, in case of failure, the whole system is replaced by a new one. Therefore, in this sense, certain systems which are physically unrepairable can be considered repairable in this context.

For a repairable system, both the up and the down times are important. To describe them we introduce two independent sets of independent random variables $U_{i}$ and $D_{i}$ for $i=1,2, \ldots$. The quantity $U_{i}$ is the length of the $i^{\text {th }}$ time interval in which the system is up and $D_{i}$ is the corresponding down time interval. We denote by $F_{i}(t)$ and $G_{i}(t)$ the distribution functions of $U_{i}$ and $D_{i}$, respectively, for all $i \geq 1$. The general case where all the functions $F_{i}(t)$ and $G_{i}(t)$ are different is very difficult to be handled analytically, as far as the reliability characteristics are concerned, which we shall discuss in this chapter. We therefore only consider the case where $F_{i}(t)=F(t)$ and $G_{i}(t)=G(t)$ for all $i \geq 1$. That means, we discuss systems which, after repair, have the same failure distribution function $F(t)$ and have the same distribution function $G(t)$ for repair any time they fail. In many practical cases we have approximately such a situation.

Instead of speaking of repair of systems or components, often the more general expression maintenance is used, which includes scheduled and unscheduled repair operations. A basic characteristic of repairable systems is the concept of maintainability, which can be defined for systems where the down times $D_{i}$ have the same distribution function $G(t)$ as we have assumed.

\section{Definition 6}

The probability $M(t)$ that a failed system is repaired within the time $[0, t]$ is called the maintainability (or maintainability function) of the system.

Naturally, the maintainability $M(t)$ is, like the reliability $R(t)$ of unrepairable systems, dependent on many conditions which have not been mentioned explicitly in Definition 6 . For instance, the maintainability is highly dependent on the repair-men and their equipment. 
Let us suppose that maintenance of the system starts immediately after the system went down. The time intervals $\mathrm{D}_{i}$ then describe the repair or maintenance times. We therefore have, since $G_{i}(t)=G(t)$ :

$$
M(t)=G(t)
$$

We will always suppose that $M(t)$ has a probability density function

$$
m(t)=M^{\prime}(t) \text {, }
$$

which is called the maintenance density function.

Analogously to the failure rate $\lambda(t)$ of an unrepairable system, we define the maintenance rate

$$
\mu(t)=\frac{m(t)}{1-M(t)} .
$$

This characteristic can intuitively be interpreted as follows: $\mu(t) \Delta t$ describes, to the first order of magnitude in $\Delta t$, the probability that the system will be repaired within the interval $[t, t+\Delta t]$ under the condition that it is not repaired at the time $t$.

We may always assume that $M(0)=0$. Therefore, using Eqs. (II.1) and (II.2) we easily obtain

$$
M(t)=1-\exp \left[-\int_{0}^{t} \mu(\tau) d \tau\right]
$$

which is analogous to formula (I.4) for the reliability $R(t)$ of an unrepairable system.

Suppose that the maintenance time $\mathrm{T}_{\mathrm{M}}$ has an expected value, which is, for instance, true if $\mu(t) \geq \mu>0$, we then call

$$
\operatorname{MTTR}=\int_{0}^{\infty} t m(t) d t .
$$

the mean time to repair which can also be expressed by

$$
\operatorname{MTTR}=\int_{0}^{\infty}[1-\mathrm{M}(\mathrm{t})] \mathrm{dt} .
$$

This characteristic describes the average time it takes to repair a system with maintenance function $M(t)$.

In practice, the maintenance rate $\mu(t)$ is mostly considered to be constant $[\mu(t)=\mu]$ even if this is not a very good model for the repair rate. In reality an increasing function $\mu(t)$ as repair rate would be more realistic. But a constant maintenance rate $\mu$ leads to relatively simple and easily manageable formulae for repairable systems. The maintainability $M(t)$ is then given by

$$
M(t)=1-e^{-\mu t}
$$


and the mean time to repair is

$$
\operatorname{MTTR}=\frac{1}{\mu}
$$

We now come to the definition of two further reliability characteristics of repairable systems.

\section{Definition ?}

The probability $R\left(t, t_{0}\right)$ that a repairable system is operational at the moment $t$ and then operates without failure, at least up to the moment $t+t_{0}$, is called the nonstationary reliability of the system. The limit

$$
R_{\infty}\left(t_{0}\right)=\lim _{t \rightarrow \infty} R\left(t, t_{0}\right)
$$

is called the stationary reliability of the system.

These concepts are generalizations of the reliability $R(t)$ of an unrepairable system. In fact, if we consider a repairable system as unrepairable, which means that we consider it from $t=0$ over the first up time period $U_{1}$, we have for the reliability $P\left(t_{0}\right)\left[=R\left(t_{0}\right)\right.$ in the notation of Chapter I] of the unrepairable system the equation

$$
P\left(t_{0}\right)=R\left(0, t_{0}\right) \text {. }
$$

Since in our case all up times $U_{i}, i=1,2, \ldots$, have the same distribution we can speak about the average time between two failures or the mean time between failure MTBF, which is given by

$$
\operatorname{MTBF}=\int_{0}^{\infty} P(t) d t=\int_{0}^{\infty} R(0, t) d t
$$

or

$$
\operatorname{MTBF}=\int_{0}^{\infty}[1-F(t)] d t
$$

Equation (II.11) is easily seen when we remember that $F(t)$ is the distribution function of the up times $U_{i}$.

How do we find $R\left(t, t_{0}\right)$ given the failure distribution function $F(t)$ and the maintenance function $M(t)$ ?

To answer this question, we first give a general expression for $R\left(t, t_{0}\right)$ :

$$
R\left(t, t_{0}\right)=\sum_{i=0}^{\infty} P\left[\sum_{j=0}^{i}\left(U_{j}+D_{j}\right)<t<t+t_{0} \leq \sum_{j=0}^{i}\left(U_{j}+D_{j}\right)+U_{i+1}\right] \text {, }
$$

where $U_{0}=D_{0}=0$. The formula (II.12) is easily seen when we introduce the following random function $X(t)$

$$
X(t)= \begin{cases}0, & \text { if the system is up, } \\ 1, & \text { if the system is down. }\end{cases}
$$


(A random function or random process, in general, is a function $Z(t)$ which is a random variable for every fixed t.) A typical sample of $X(t)$ is shown in Fig. 24. It gives us an idea of how to show Eq. (II.12). In fact, the quantity $R\left(t, t_{0}\right)$ is the probability that the interval $\left(t, t+t_{0}\right)$ is included in either $U_{1}$ or $U_{2}$ and so on, and this fact is expressed by the right-hand side of Eq. (II.12).

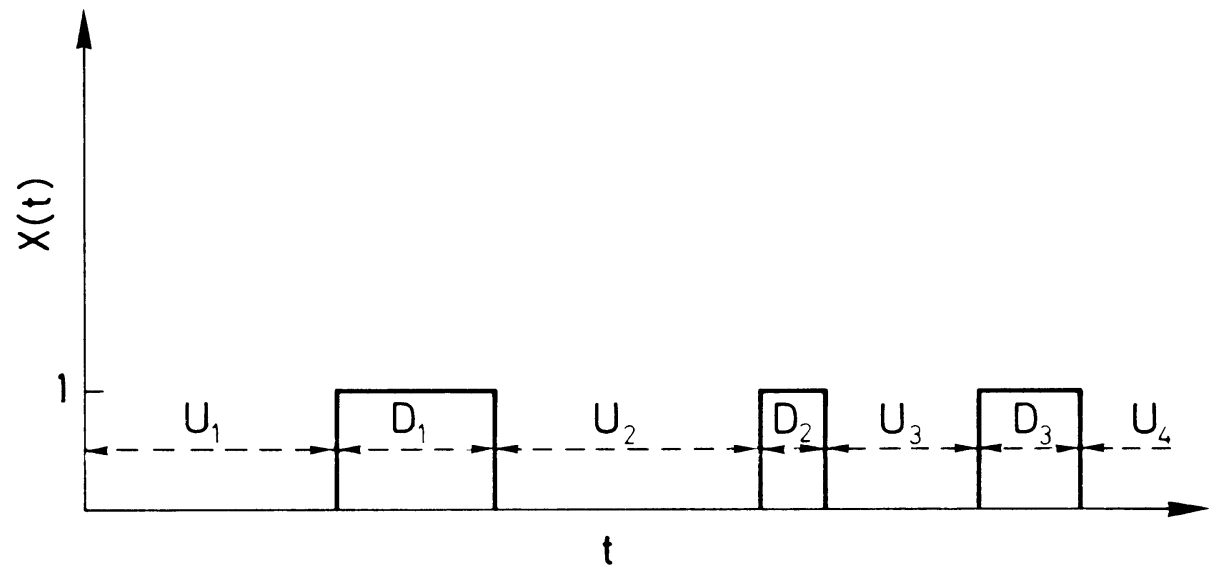

Fig. 24 Sample of $x(t)$

Using methods from probability theory which are beyond the scope of this introductory report, one can show that the right-hand side of Eq. (II.12) is given by

$$
R\left(t, t_{0}\right)=P\left(t+t_{0}\right)+\int_{0}^{t} P\left(t+t_{0}-\tau\right) k(\tau) d \tau,
$$

where $P(t)=1-F(t)$ is the reliability of the system considered as an unrepairable system and

$$
\begin{aligned}
& k(t)=\sum_{i=1}^{\infty} k_{i}(t), \\
& k_{i}(t)=\int_{0}^{t} k_{i^{\prime}-1}(t-\tau) k_{1}(\tau) d \tau, \quad i=2,3, \ldots, \\
& k_{1}(t)=\int_{0}^{t} f(t-\tau) m(\tau) d \tau \\
& f(t)=F^{\prime}(t), \quad m(t)=M^{\prime}(t) .
\end{aligned}
$$

Equation (II.14) answers the question which we posed on page 37. Naturally, it is a problem to find $k(t)$ in Eq. (II.15) in practice. For the special case where the $U_{i}$ and the $D_{i}$ both have an exponential distribution with parameters $\lambda$ and $\mu$, respectively, it can be shown by the aid of the Laplace transform $\left.^{8}, 9\right)$ that

$$
k(t)=\frac{\lambda \mu}{\lambda+\mu}\left[1-e^{-(\lambda+\mu) t}\right]
$$


Since

$$
P\left(t_{0}\right)=e^{-\lambda t_{0}}
$$

in this case, we finally find from Eqs. (II.14) to (II.19):

$$
R\left(t, t_{0}\right)=\left[\frac{\mu}{\lambda+\mu}+\frac{\lambda}{\lambda+\mu} e^{-(\lambda+\mu) t}\right] e^{-\lambda t_{0}} .
$$

From this expression it is easy to obtain the stationary reliability $R_{\infty}\left(t_{0}\right)$ defined in Eq. (II.8):

$$
R_{\infty}\left(t_{0}\right)=\frac{\mu}{\lambda+\mu} e^{-\lambda t_{0}}
$$

We compare $R_{\infty}\left(t_{0}\right)$ in this equation with the reliability $P\left(t_{0}\right)$ of the system considered as an unrepairable system given in Eq. (II.19) and we see that

$$
\mathrm{R}_{\infty}\left(\mathrm{t}_{0}\right)=\frac{\mu}{\lambda+\mu} \mathrm{P}\left(\mathrm{t}_{0}\right)
$$

which shows that, in the exponential case, the stationary reliability $R_{\infty}\left(t_{0}\right)$ is equal to the reliability $P\left(t_{0}\right)$ of the corresponding unrepairable system times a constant reduction factor. This factor tends to one for $\mu \rightarrow \infty$ or $1 / \mu=$ MTTR $\rightarrow 0$. That means $R_{\infty}\left(t_{0}\right)$ tends to $P\left(t_{0}\right)$ for MTTR $\rightarrow 0$.

Equation (II.22) gives the stationary reliability of a repairable system with exponential failure and maintenance distributions. For the general case it can be $\mathrm{shown}^{2}$ ) that we have

$$
R_{\infty}\left(t_{0}\right)=\frac{1}{M T B F+M T T R} \int_{t_{0}}^{\infty} P(\tau) d \tau,
$$

where $P(t)=1-F(t)$ is the reliability function. We can see from Eq. (II.23) that the dependence of $R_{\infty}\left(t_{0}\right)$ on the maintenance distribution only appears in the form of the mean time to repair MTTR. This is very important, since it is much easier to obtain information about the MTTR rather than about the whole distribution. This is even more important, since in practice we are much more interested in the stationary reliability $R_{\infty}\left(t_{0}\right)$ than in the non-stationary reliability $R\left(t, t_{0}\right)$.

Let us now summarize our results about the characteristics $R\left(t, t_{0}\right)$ and $R_{\infty}\left(t_{0}\right)$.

Theorem 8

Suppose a repairable system has the reliability function $P(t)=1-F(t)$, the failure density $f(t)$ and a maintenance law with density $m(t)$. Then, the non-stationary reliability $R\left(t, t_{0}\right)$ of the system is given by formula (II.14) and the corresponding stationary reliability $R_{\infty}\left(t_{0}\right)$ is given by Eq. (II.23). In the case of exponential failure and maintenance distributions, Eqs. (II.20) and (II.21) yield the corresponding quantities.

We now come to the last two reliability characteristics for repairable systems which we discuss here. 


\section{Definition 8}

The probability $A(t)$ that a repairable system is operational at the moment $t$ is called the non-stationary availability of the system. The limit

$$
\mathrm{A}_{\infty}=\lim _{\mathrm{t} \rightarrow \infty} \mathrm{A}(\mathrm{t})
$$

is called the stationary availability of the system.

Both characteristics given in Definition 8 are special cases of the non-stationary and the stationary reliability, respectively, but their intuitive interpretation justifies the introduction of special names for these characteristics. In fact, we have

$$
\begin{aligned}
A(t) & =R(t, 0), \\
A_{\infty} & =R_{\infty}(0) .
\end{aligned}
$$

We can therefore immediately formulate the following theorem.

\section{Theorem 9}

Under the conditions of Theorem 8, the non-stationary availability of a repairable system is given by

$$
A(t)=P(t)+\int_{0}^{t} P(t-\tau) k(\tau) d \tau
$$

and the stationary availability is given by

$$
\mathrm{A}_{\infty}=\frac{\mathrm{MTBF}}{\mathrm{MTBF}+\mathrm{MTTR}} \text {. }
$$

In the case of exponential failure and maintenance distributions we have

$$
\begin{aligned}
A(t) & =A_{\infty}+\frac{\lambda}{\lambda+\mu} e^{-(\lambda+\mu) t} \\
A_{\infty} & =\frac{\mu}{\lambda+\mu} .
\end{aligned}
$$

Using formula (II.28) for the non-stationary availability, which immediately follows from Eq. (II.23), one often writes Eq. (II.23) in the form

$$
R_{\infty}\left(t_{0}\right)=\frac{A_{\infty}}{M T B F} \int_{t_{0}}^{\infty} P(\tau) d \tau,
$$

which leads, for the exponential case of formula (II.21), to the form

$$
R_{\infty}\left(t_{0}\right)=A_{\infty} e^{-\lambda \tau_{0}} \text {. }
$$


We mentioned on page 39 that in the exponential case the stationary reliability $R_{\infty}\left(t_{0}\right)$ is equal to the reliability $\mathrm{P}\left(\mathrm{t}_{0}\right)$ of the corresponding unrepairable systems times a constant reduction factor. From formula (II.32) we see that this reduction factor is exactly the stationary availability of the repairable system.

In the literature on reliability, very often only the formula (II.28) is given as a measure of availability of a repairable system. Since $A_{\infty}$ becomes larger if the mean time to repair becomes smaller, we can see that $A_{\infty}$ is a reasonable measure of availability of a repairable system, but it is only valid, analogous to the stationary reliability $R_{\infty}\left(t_{0}\right)$, for large running times $t$, which, however, describes exactly situations in which engineers are mostly interested.

We finish this section by giving a simple numerical example. Suppose we have the case of exponential failure and maintenance distributions with

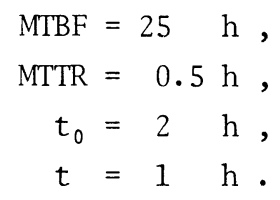

What are the values of $A_{\infty}, R_{\infty}\left(t_{0}\right), A(t)$, and $R\left(t, t_{0}\right)$ ? From the formulae (II.28), (II.32), (II.29) and (II.20) we obtain

$$
\begin{aligned}
\mathrm{A}_{0} & =0.9804, \\
\mathrm{R}_{\infty}\left(\mathrm{t}_{0}\right) & =0.905, \\
\mathrm{~A}(\mathrm{t}) & =0.9829, \\
\mathrm{R}\left(\mathrm{t}, \mathrm{t}_{0}\right) & =0.9073 .
\end{aligned}
$$

We can say that after a long running time the system is available for $98 \%$ of the time. The reliability after a long running period is $90.5 \%$ for a two-hour operation. We have a bit more than $98^{\circ}$ probability that the system is available one hour after starting. Finally, there is $90.7 \%$ probability that the system will operate failure-free for two more hours if it is operating one hour after starting.

\section{SERIES CONNECTIONS}

From Chapter I we already know what a series connection of $n$ components $C_{1}, \ldots, C_{n}$ is. It was easy to find the basic reliability characteristics of a series connection in the case of unrepairable components. If the components are repairable it is much more complicated to find the reliability characteristics for a series connection S. For a system $\mathrm{S}$ where the individual components have arbitrary failure distributions, the formulae become so complicated that they are essentially inapplicable in practice. In such cases only Monte Carlo simulations ${ }^{1,10}$ ) of the system's reliability behaviour can lead to practical solutions. We shall therefore consider a more restricted model which uses methods applied in Queuing Theory ${ }^{11}$ ).

Before we formulate the assumptions for our model, we recall to the reader the meaning of the symbol $o(x)$. We say that a function $f(x)$ is of the order $o(x)$ for $x \rightarrow 0$ if $f(x) / x \rightarrow 0$ for $x \rightarrow 0$. As an example we have

$$
\mathrm{P}(\Delta \mathrm{t})=1-\mathrm{e}^{-\lambda \Delta \mathrm{t}}=\lambda \Delta \mathrm{t}-\frac{\lambda^{2}}{2}(\Delta \mathrm{t})^{2}+\ldots=\lambda \Delta \mathrm{t}+\mathrm{o}(\Delta \mathrm{t})
$$


For our considered model of a repairable system we make the following assumptions:

a) The components $C_{1}, \ldots, C_{n}$ of the series connection $S$ have independent lifetimes.

b) The probability that two or more components fail at the same time is zero.

c) When one of the components fails the remaining components are switched off until the failed component is repaired.

d) The probability $L_{i}$ that the component $C_{i}$ fails within $(t, t+\Delta t)$ under the condition that $C_{i}$ is operating at time $t$ is given by

$$
\mathrm{L}_{\mathrm{i}}=\lambda_{\mathrm{i}} \Delta \mathrm{t}+\mathrm{o}(\Delta \mathrm{t}), \quad \mathrm{i}=1,2, \ldots, \mathrm{n} .
$$

e) The probability $M_{i}$ that the component $C_{i}$ is repaired within $(t, t+\Delta t)$ under the condition that $C_{i}$ is not yet repaired at time $t$ is given by

$$
\mathrm{N}_{\mathrm{i}}=\mu_{\mathrm{i}} \Delta \mathrm{t}+\mathrm{o}(\Delta \mathrm{t}), \quad \mathrm{i}=1,2, \ldots, \mathrm{n} .
$$

Given the assumptions (a) to (e) we now want to find the reliability characteristics for the series connection $S$ of the repairable components $C_{1}, \ldots, C_{n}$.

Let us first briefly discuss the assumptions (a) to (e). The point (a) is the same as in Chapter I. Assumption (b) is not really an assumption, since we always assume that the lifetimes of the components have a continuous distribution. Assumption (c) will not always be satisfied in practice. We could also consider the case where the remaining components stay in operational state. For convenience we confine ourselves to the case given in assumption (c). The other case is treated in Ref. 2. The assumptions (d) and (e) define the so-called transition probabilities $\mathrm{L}_{i}$ and $\mathrm{M}_{i}$, respectively, for the transition of the components from the state "up" to the state "down" and vice versa, respectively. The formulae (II.34) and (II.35) essentially say that our components have exponential failure and maintenance distributions with failure rates $\lambda_{i}$ and maintenance rates $\mu_{i}$, respectively. The reason why the exponentiality of the corresponding distributions is expressed as in the assumptions (d) and (e) will become clear later.

The series connection $S$ can be described in the following way. We say that $S$ is in the state

$\mathrm{H}_{0}$, if $\mathrm{S}$ is up,

$\mathrm{H}_{i}$, if $\mathrm{C}_{i}$ is down and all $\mathrm{C}_{j}, j \neq i$, are up for $i=1,2, \ldots, n$.

Because of the assumption (b) we then know that the system $S$ is (with probability 1) in exactly one of the states $H_{j}, j=0,1, \ldots, n$, at any moment $t$. Suppose we denote by $P_{j}(t), j=0,1, \ldots, n$, the probability that the system $S$ is in state $H_{j}$ at the moment $t$. We shall then see that we can determine the reliability characteristics

$$
\begin{aligned}
& P(t) \quad=1-F(t)=\text { reliability function of } S \text { (S considered unrepairable), } \\
& M(t) \quad=\text { maintenance function of } S, \\
& A(t) \quad=\text { non-stationary availability of } S, \\
& R\left(t, t_{0}\right)=\text { non-stationary reliability of } S,
\end{aligned}
$$

and the corresponding derived characteristics like MTBF, MTTR, $A_{\infty}$ and $R_{\infty}\left(t_{0}\right)$, if we know 
$\mathrm{P}_{0}(t)$ for three different "configurations" of the system $\mathrm{S}$. By the "configurations" of the system $\mathrm{S}$ we mean the following three different interpretations of the system $\mathrm{S}$.

Configuration 1: S begins to run at $t=0$ and is considered to be unrepairable.

Configuration 2: S went down at $t=0$ and is observed until it is repaired.

Configuration 3: $S$ begins to run at $t=0$ and is considered to be repairable.

It is obvious that the probabilities $P_{j}(t)$ for $j=0,1, \ldots, n$, of the possible states of $\mathrm{S}$ will be dependent on the different configurations in which $\mathrm{S}$ will be. We therefore have to introduce three different sets of state probabilities

$$
P_{1 j}(t), \quad P_{2 j}(t), \quad P_{3 j}(t), \quad j=0,1, \ldots, n,
$$

corresponding to the three different configurations of $\mathrm{S}$.

We now ask what the advantage of introducing these probabilities is for us. The answer is given by the following formulae:

$$
\begin{aligned}
P(t) & =P_{10}(t), \\
M(t) & =P_{20}(t), \\
A(t) & =P_{30}(t), \\
R\left(t, t_{0}\right) & =P_{10}\left(t+t_{0}\right)+\int_{0}^{t} P_{10}\left(t+t_{0}-\tau\right) k(\tau) d \tau, \\
M T B F & =\int_{0}^{\infty} P_{10}(t) d t, \\
M T T R & =\int_{0}^{\infty}\left[1-P_{20}(t)\right] d t, \\
A_{\infty} & =\lim _{t \rightarrow \infty} P_{30}(t), \\
R\left(t_{0}\right) & =\frac{A_{\infty}}{M T B F} \int_{t_{0}}^{\infty} P_{10}(t) d t
\end{aligned}
$$

where in formula (II.39) the function $k(t)$ is given by

$$
\begin{aligned}
k(t) & =\sum_{i=1}^{\infty} k_{i}(t), \\
k_{1}(t) & =-\int_{0}^{t} P_{10}^{\prime}(t-\tau) P_{20}^{\prime}(\tau) d \tau, \\
k_{i+1}(t) & =\int_{0}^{t} k_{i}(t-\tau) k_{1}(\tau) d \tau, \quad i=2,3, \ldots .
\end{aligned}
$$


The formulae (II.36) to (II.46) are easily found when the definitions of the different configurations are taken into account and the formulae of Theorems 8 and 9 are used.

Looking at a11 the expressions in Eqs. (II.36) to (II.46), we observe that all the reliability characteristics for our system $S$ are known if $F_{10}(t), P_{20}(t)$ and $P_{30}(t)$ are known. We therefore pose the next question: "How to determine $P_{10}(t), P_{20}(t)$ and $P_{30}(t)$ ?" The answer is: "Each set of state probabilities $P_{1 j}(t), P_{2 j}(t)$, and $P_{3 j}(t)$ for $j=0,1, \ldots, n$, is a solution of a different set of simultaneous ordinary differential equations with constant coefficients." We shall therefore derive these differential equations and try to find their solutions. It is, in general, not possible to find differential equations for the quantities $P_{i 0}(t), i=1,2,3$, alone. In other words, for fixed $i$ (= configuration $i$ ) the system of differential equations is coupled with respect to the index $j$, at least for the configurations 2 and 3 . To show how the mentioned systems of differential equations are derived, we will discuss configuration 1 in the special case of $n=1$. The derivation of the corresponding system of differential equations will show us the principle of how to derive the equations for all configurations and for any $\mathrm{n} \geq 1$.

Let us now consider configuration 1 for $n=1$ (i.e. $S$ has only one component). We use the following notation

$$
\begin{aligned}
P_{1 j}\left(t+\Delta t \mid H_{k}\right)= & \text { probability that the system goes to state } H_{j} \text { during }[t, t+\Delta t] \text { under } \\
& \text { the condition that it is in state } H_{k} \text { at time } t .
\end{aligned}
$$

Using the concept of conditional probability it is easily seen that

$$
\begin{aligned}
& P_{10}(t+\Delta t)=P_{10}\left(t+\Delta t \mid H_{0}\right) P_{10}(t)+P_{10}\left(t+\Delta t \mid H_{1}\right) P_{11}(t), \\
& P_{11}(t+\Delta t)=P_{11}\left(t+\Delta t \mid H_{0}\right) P_{10}(t)+P_{11}\left(t+\Delta t \mid H_{1}\right) P_{11}(t) .
\end{aligned}
$$

It is easy to interpret these equations intuitively. We take, for instance, the first equation in (II.47). On the left-hand side we have the probability that the system $S$ (configuration 1 ) is in state $H_{0}$ at time $t+\Delta t$. This must be equal to the probability $\mathrm{P}_{10}(\mathrm{t})$ that $\mathrm{S}$ is in $\mathrm{H}_{0}$ at time $t$ times the probability $\mathrm{P}_{10}\left(\mathrm{t}+\Delta \mathrm{t} \mid \mathrm{H}_{0}\right)$ that $\mathrm{S}$ is in $\mathrm{H}_{0}$ at time $t$ and that it stays there during $[t, t+\Delta t]$ plus the probability $P_{11}(t)$ that $S$ is in $H_{1}$ at time $t$ times the probability $P_{10}\left[t+\Delta t \mid H_{1}\right]$ that $S$ is in $H_{1}$ at time $t$ and that it changes to $H_{0}$ during $[t, t+\Delta t]$.

The generalization of the system (II.47) for any number $n \geq 1$ of components and any configuration $i=1,2,3$ is given by

$$
P_{i j}(t+\Delta t)=\sum_{k=0}^{n} P_{i j}\left(t+\Delta t \mid H_{k}\right) P_{i k}(t)
$$

for $j=0,1, \ldots, n$ and $i=1,2,3$.

For the derivation of the system of differential equations for the system $\mathrm{S}$ with one component in configuration 1 , we use the system (II.47). In this system we know the transition probabilities $P_{1 j}\left(t+\Delta t \mid H_{k}\right)$ for $j=0,1$, and $k=0,1$. We have

$$
\mathrm{P}_{10}\left(\mathrm{t}+\Delta \mathrm{t} \mid \mathrm{H}_{1}\right)=0
$$




$$
\begin{aligned}
& \mathrm{P}_{11}\left(\mathrm{t}+\Delta \mathrm{t} \mid \mathrm{H}_{1}\right)=1, \\
& \mathrm{P}_{11}\left(\mathrm{t}+\Delta \mathrm{t} \mid \mathrm{H}_{0}\right)=\lambda_{1} \Delta \mathrm{t}+\mathrm{o}(\Delta \mathrm{t}), \\
& \mathrm{P}_{10}\left(\mathrm{t}+\Delta \mathrm{t} \mid \mathrm{H}_{0}\right)=1-\left[\lambda_{1} \Delta \mathrm{t}+\mathrm{o}(\Delta \mathrm{t})\right] .
\end{aligned}
$$

To see why the formulae (II.49) to (II.52) are true we have to remember that our system is in configuration 1 and that we have the assumption (d) on page 42. Equation (II.49) then expresses that $\mathrm{S}$ will never come back to state $\mathrm{H}_{0}$, and formula (II.50) expresses the same by saying that $\mathrm{S}$ will always stay in $\mathrm{H}_{1}$ if it is there once. Equation (II.51) gives, according to assumption (d), the transition probability from $H_{0}$ to $H_{1}$ and formula (II.52) expresses the probability that $S$ will stay in $H_{0}$ which must be equal to $1-P_{11}\left(t+\Delta t \mid H_{0}\right)$ since the system can only be either in $\mathrm{H}_{0}$ or in $\mathrm{H}_{1}$.

We now insert Eqs. (II.49) to (II.52) into the system (II.47) and after some trivial manipulations we end up with the system

$$
\begin{aligned}
& \frac{P_{10}(t+\Delta t)-P_{10}(t)}{\Delta t}=-\lambda_{1} P_{10}(t)+\frac{1}{\Delta t} o(\Delta t), \\
& \frac{P_{11}(t+\Delta t)-P_{11}(t)}{\Delta t}=\lambda_{1} P_{10}(t)+\frac{1}{\Delta t} o(\Delta t) .
\end{aligned}
$$

In the system (II.53) we take $\Delta t \rightarrow 0$ and obtain, since $(1 / \Delta t) \circ(\Delta t) \rightarrow 0:$

$$
\begin{aligned}
& P_{10}^{\prime}(t)=-\lambda_{1} P_{10}(t), \\
& P_{11}^{\prime}(t)=\lambda_{1} P_{10}(t) .
\end{aligned}
$$

With Eqs. (II.54) we found the previously announced system of differential equations for $S$ in configuration 1 and with $n=1$. To find a unique solution of the system we need the corresponding initial conditions. Since $S$ is in configuration 1 it is easily seen that we have

$$
\begin{aligned}
& P_{10}(0)=1, \\
& P_{11}(0)=0 .
\end{aligned}
$$

We now return to the general case of $n$ components and to all configurations 1,2 , and 3 . Using the system of equations (II.48), the assumptions (d) and (e) on page 42 and the definitions of the different configurations 1,2 , and 3 on page 43 , one arrives, in an analogous way as for the special case, at the following systems of differential equations together with the corresponding initial conditions $(i=1,2, \ldots, n)$ :

$$
\begin{aligned}
& P_{10}^{\prime}(t)=-\Lambda P_{10}(t), \\
& P_{1 i}^{\prime}(t)=\lambda_{i} P_{10}(t), \\
& P_{10}(0)=1, \\
& P_{1 i}(0)=0
\end{aligned}
$$




$$
\begin{aligned}
-46- & \\
\Lambda & =\sum_{i=1}^{n} \lambda_{i}, \\
P_{20}^{\prime}(t) & =\sum_{i=1}^{n} \mu_{i} P_{2 i}(t), \\
P_{2 i}^{\prime}(t) & =-\mu_{i} P_{2 i}(t), \\
P_{20}(0) & =0, \\
P_{2 i}(0) & =\frac{\lambda_{i}}{\Lambda}, \\
P_{30}^{\prime}(t) & =-\Lambda P_{30}(t)+\sum_{i=1}^{n} \mu_{i} P_{3 i}(t), \\
P_{3 i}(0) & =0 . \\
P_{3 i}^{\prime}(t) & =\lambda_{i} P_{30}(t)-\mu_{i} P_{3 i}(t), \\
& =1,
\end{aligned}
$$

[We might mention that it is not trivial to show that $P_{2 i}(0)=\lambda_{i} \Lambda^{-1}$ in Eq. (II.60), but it is intuitively clear.]

We now have to solve the above three systems of differential equations in order to find the functions $P_{10}(t), P_{20}(t)$, and $P_{30}(t)$ which we need for our reliability characteristics in Eqs. (II.36) to (II.46).

Since the system (II.56) is decoupled we immediately find $P_{10}(t)$ from the system (II.56):

$$
P_{10}(t)=e^{-\Lambda t}
$$

In the system (II.59) we easily find $\mathrm{P}_{2 i}(t)$ as

$$
P_{2 i}(t)=\frac{\lambda_{i}}{\Lambda} e^{-\mu_{i} t}, \quad i=1,2, \ldots, n
$$

Since we have

$$
\mathrm{P}_{20}(\mathrm{t})=1-\sum_{\mathrm{i}=1}^{\mathrm{n}} \mathrm{P}_{2 \mathrm{i}}(\mathrm{t}),
$$

we finally obtain

$$
\mathrm{P}_{20}(\mathrm{t})=1-\sum_{\mathrm{i}=1}^{\mathrm{n}} \frac{\lambda_{i}}{\Lambda} \mathrm{e}^{-\mu_{\mathrm{i}} \mathrm{t}}
$$


It is more complicated to solve the system (II.61) . He apply the method of the Laplace transform. Let

$$
p_{j}(s)=\int_{0}^{\infty} e^{-s t} P_{3 j}(t) d t
$$

for $j=0,1, \ldots, n$ be the Laplace transform of $P_{3 j}(t)$. Applying formula (II.66) to Eqs. (II.61) and using Eq. (II.62) we obtain

$$
\begin{aligned}
& s \mathrm{p}_{0}(\mathrm{~s})-1=-\Lambda \mathrm{p}_{0}(\mathrm{~s})+\sum_{\mathrm{i}=1}^{\mathrm{n}} \mu_{\mathrm{i}} \mathrm{p}_{\mathrm{i}}(\mathrm{s}) \\
& \mathrm{s} \mathrm{p}_{\mathrm{i}}(\mathrm{s})=\lambda_{\mathrm{i}} \mathrm{p}_{0}(\mathrm{~s})-\mu_{\mathrm{i}} \mathrm{p}_{\mathrm{i}}(\mathrm{s})
\end{aligned}
$$

for $i=1,2, \ldots, n$. This is a system of linear algebraic equations for the unknown functions $p_{j}(s)$. It is easy to check that the solution to the system (II.67) is given by

$$
\begin{aligned}
& p_{0}(s)=\frac{1}{s\left(1+\sum_{k=1}^{n} \frac{\lambda_{k}}{s+\mu_{k}}\right)} \\
& p_{i}(s)=\frac{\lambda_{i}}{s+\mu_{i}} p_{0}(s), \quad i=1,2, \ldots, n .
\end{aligned}
$$

Since the function $\mathrm{p}_{0}(\mathrm{~s})$ given in Eq. (II.68) is the Laplace transform of the function $\mathrm{P}_{30}(\mathrm{t})$, we only have to find the original function to $\mathrm{p}_{0}(\mathrm{~s})$ in a table for the Laplace transform. Unfortunately, there exists no general formula for the original function $\mathrm{P}_{30}(\mathrm{t})$ of $\mathrm{p}_{0}(\mathrm{~s})$ if the $\mu_{i}$ are distinct. However, in the special case where $\mu_{i}=\mu$ for $i=1,2, \ldots, n$, we can write $p_{0}(s)$ in the form

$$
\mathrm{p}_{0}(\mathrm{~s})=\frac{\mu}{(\Lambda+\mu)} \frac{1}{\mathrm{~s}}+\frac{\Lambda}{(\Lambda+\mu)} \frac{1}{(\mathrm{~s}+\Lambda+\mu)},
$$

where $\Lambda$ is given by Eq. (II.58). From this equation we easily find

$$
\mathrm{P}_{30}(\mathrm{t})=\frac{\mu}{\Lambda+\mu}+\frac{\Lambda}{\Lambda+\mu} \mathrm{e}^{-(\Lambda+\mu) \mathrm{t}}
$$

for $\mu_{i}=\mu, i=1, \ldots, n$.

We now know the functions $\mathrm{P}_{10}(t), \mathrm{P}_{20}(t)$, and $\mathrm{P}_{30}(t)$, where in the latter case we know the explicit form of the function only for $\mu_{i}=\mu, i=1,2, \ldots$, n. If the $\mu_{i}$ are distinct we know the Laplace transform (II.68) of $\mathrm{P}_{30}(\mathrm{t})$ which has to be evaluated numerically, that means the zeros of the denominator have to be found in each particular case. Inserting the formulae (II.63), (II.65), and (II.71) into Eqs. (II.36) to (II.46), we finally obtain

$$
\mathrm{P}(\mathrm{t})=\mathrm{e}^{-\Lambda \mathrm{t}}, \quad \Lambda=\sum_{\mathrm{i}=1}^{\mathrm{n}} \lambda_{\mathrm{i}},
$$




$$
\begin{aligned}
M(t) & =1-\sum_{i=1}^{n} \frac{\lambda_{i}}{\Lambda} e^{-\mu_{i} t}, \\
A(t) & =\frac{\mu}{\Lambda+\mu}+\frac{\Lambda}{\Lambda+\mu} e^{-(\Lambda+\mu) t} \quad\left(\mu_{i}=\mu, i=1, \ldots, n\right) . \\
R\left(t, t_{0}\right) & =A(t) e^{-\Lambda t_{0}} \quad\left(\mu_{i}=\mu, i=1, \ldots, n\right), \\
\operatorname{MTBF} & =\frac{1}{\Lambda} \\
\operatorname{MTTR} & =\frac{1}{\Lambda} \sum_{i=1}^{n} \frac{\lambda_{i}}{\mu_{i}}, \\
A_{\infty} & =\frac{1}{1+\sum_{i=1}^{n} \frac{\lambda_{i}}{\mu_{i}}} \\
R_{\infty}\left(t_{0}\right) & =A_{\infty} e^{-\Lambda t_{0}} .
\end{aligned}
$$

We have to say a word on how we found $\mathrm{A}_{\infty}$ in Eq. (II. 78). In the theory of the Laplace transform $\left.{ }^{8}, 9\right)$, it is shown that

$$
A_{\infty}=\lim _{s \rightarrow 0} s p_{0}(s)
$$

which immediately yields the formula (II.78) and which is valid for any positive $\mu_{i}$, $i=1, \ldots, n$.

We now summarize the results of this section in the following theorem.

Theorem 10

Suppose we have a series connection $S$ of independent repairable components $C_{1}, \ldots, C_{n}$, the assumptions (a) to (e) on page 42 are satisfied and the down times $D_{k}$ have the same distribution function $M(t)$ for $k=1,2, \ldots$. Then, the up times $U_{k}$ have the same distribution function $F(t)$ for $k=1,2, \ldots$, and the reliability characteristics $P(t)$, $M(t), A(t), R\left(t, t_{0}\right), M T B F, M T T R, A_{\infty}$, and $R_{\infty}\left(t_{0}\right)$ are given by Eqs. (II.72) to (II.79).

For completeness we must say that, as is stated in this theorem, the identical distribution of the up times $U_{k}$ follows from the fact that systems with exponentially distributed lifetimes do not age (shown in Chapter I, pages 9 and 10).

We finish this section with a numerical example. We consider a series connection of 100 equal repairable components each having

$$
\begin{aligned}
& \text { MTBF }=100000 \mathrm{~h}, \\
& \text { MTTR }=5 \mathrm{~h} .
\end{aligned}
$$


We want to calculate the characteristics (II.72) to (II.79) for that system taking $t=t_{0}=10 \mathrm{~h}$. The result is:

$$
\begin{aligned}
\mathrm{P}(10) & =0.990, & \mathrm{MTBF} & =1000 \mathrm{~h}, \\
\mathrm{M}(10) & =0.865, & \mathrm{MTTR} & =5 \mathrm{~h}, \\
\mathrm{~A}(10) & =0.996, & \mathrm{~A}_{\infty} & =0.995, \\
\mathrm{R}(10,10) & =0.986, & \mathrm{R}_{\infty}(10) & =0.985 .
\end{aligned}
$$

Remark: In this section we provided the basic mathematical tools which are normally also used to treat repairable exponentially distributed systems with redundancies. But the formulae for such systems are much more involved. We therefore skip the discussion of redundant repairable systems. The interested reader, however, should be able, after a careful study of this section, to understand the corresponding literature about redundant repairable systems (see Ref. 2). In addition, we might mention again that in most of the complicated practical cases only a Monte Carlo simulation of the reliability behaviour of the system will lead to practical solutions. A discussion of these methods, however, is beyond the scope of this report. The interested reader may find an example in Ref. 1.

\section{COMPUTER PROGRAM TO MON ITOR THE MAINTENANCE ON THE CDC 6600}

In this section we want to discuss briefly an existing computer program which monitors maintenance operations on the CDC 6600 computer. The discussion of this program should show the reader how some of the reliability characteristics which have been defined for repairable systems in this chapter are used in practice and how others, which have not been defined here, may be introduced and applied.

The program is called MNT and was developed by the Air Force Weapons Laboratory (AFWL), USA, in 1970. The program basically provides per computer component the

- mean time to response (MTRR)

- mean time to failure (MTTF)

- mean time to repair (MTTR)

- per cent up time (PCTUP)

- failure rate (FR)

- reliability for a prescribed time (R)

- confidence intervals for MTTF, FR and R.

The mentioned characteristics are defined as follows:

The response time is the difference in time between notification of component failure and arrival of customer engineer who is responsible for the repair.

The time to failure is the difference in time between the time down for the current failure and time up from the last failure.

The time to repair is the difference in time between completion time on this call and arrival time on this call.

The time up is the difference between the total operating hours and total hours down. 
The failure distribution of the components is supposed to be exponential. Therefore, the failure rate FR is assumed constant. The confidence intervals for MTTF, FR and R provide a statistical means to control, for instance, each time a data evaluation by the program MNT is made, whether this assumption stays true, or whether the failure rate is increasing. [A more detailed discussion of the concept of a confidence interval can be found in every textbook on statistics, for instance, in Guttman and Wilks $\left.\left.{ }^{12}\right)\right]$.

For the characteristics MTRR, MTTF, MTTR, and PCTUP, the following approximate values are used in the program MNT:

$$
\begin{aligned}
& \text { MTRR }=\frac{1}{k} \sum_{i=1}^{k}\left(\mathrm{AT}_{\mathrm{i}}-\mathrm{NT}_{\mathrm{i}}\right), \\
& \mathrm{MTTF}=\frac{1}{\mathrm{n}} \sum_{i=1}^{\mathrm{n}}\left(\mathrm{CTD}_{\mathrm{i}}-\mathrm{LTU}_{\mathrm{i}}\right), \\
& \text { MTTR }=\frac{1}{\mathrm{n}} \sum_{i=1}^{\mathrm{n}}\left(\mathrm{CT}_{\mathrm{i}}-\mathrm{AT}_{\mathrm{i}}\right), \\
& \text { PCTUP }=\frac{100}{\text { OPER }} \sum_{\mathrm{i}=1}^{\mathrm{n}}\left(\mathrm{OPER}-\mathrm{HD}_{\mathrm{i}}\right),
\end{aligned}
$$

where the symbols used mean:

$$
\begin{aligned}
& \mathrm{k} \quad=\text { total number of calls on all components, } \\
& \mathrm{n} \quad=\text { number of failures of this component, } \\
& \mathrm{AT} \quad=\text { arrival time of the customer engineer, } \\
& \mathrm{NT} \quad=\text { notification time of failure, } \\
& \mathrm{CTD}=\text { current time down of this component, } \\
& \text { LTU = last time up of this component, } \\
& \mathrm{CT}=\text { completion time, } \\
& \mathrm{OPER}=\text { total operating hours of this component, } \\
& \mathrm{HD} \quad=\text { hours down of this component. }
\end{aligned}
$$

To compute by the program MNT the quantities MTRR, MTTF, MTTR, PCTUP, FR, R and the corresponding confidence intervals for every component of the CDC 6600 computer, a11 the above-mentioned data have to be provided for every component. This is technically done by filling out for every component a so-called maintenance card on which the arrival time, the notification time, and so on, appear. The information on these cards is used as the input data for the program MNT, which on return will give a list of the mentioned reliability characteristics for every considered component. These output data will then give useful information on the maintenance operation of the computer system. 


\section{OPTIMIZATION PROBLEMS}

\section{INTRODUCTORY REMARKS}

In this chapter we want to discuss briefly what kind of optimization problems occur in reliability theory, how they may be formulated in a mathematical way, and how even the process of formulating the problems may occasionally help to clarify things and to improve a system's design. We shall not discuss what the methods are to solve such problems analytically or numerically. This is a subject which must be handled separately, since it is too vast a field.

We shall basically discuss a few typical examples which principally occur in systems design:

- redundancy optimization,

- optimum number of spare parts,

- optimum time interval for age replacement.

\section{OPTIMIZATION}

Everybody who designs a system practically wants to design an "optimal" system. But what does "optimal" mean? If we do not specify, we normally mean that the system must be, for example, very reliable, to a high degree available, not too heavy, not too voluminous, not too expensive, etc. All these criteria like reliability, availability, weight, volume, cost, and many others, may describe somehow what we mean by an "optimal" system. We suppose, therefore, that we have a list of such criteria for a system which is to be designed. In addition, we assume that we know the system or design variables $x_{1}, x_{2}, \ldots, x_{n}$, of whose values we can freely dispose when designing the system. For instance, the number of components of a special type in series or in parallel connection are system variables. In other words, we suppose that the system is designed when the values of the system variables are chosen. We may then define in an intuitive way what we mean by the design of an "optimal" system as follows: "Choose the system variable values such that the system is 'best' with respect to all criteria which are taken into account."

How to do it? It is difficult since the criteria may be conflicting with, and dependent on, each other. For instance, increasing reliability implies increasing cost or weight or volume.

One approach to solve our optimization problem is the so-called Operations Research (OR) approach. It can, in principle, be applied if all relevant criteria can be quantified. 
In cases where this is not satisfied, other methods must be applied ${ }^{13,14)}$. Criteria which are difficult to be quantified are, for instance, readability, styling, prestige, and so on. Fortunately, most of the criteria which occur in connection with reliability optimization are quantifiable such that the OR approach is, in principle, applicable.

We formulate the OR approach to an optimization problem in the following form.

\section{OR Optimization Principle}

a) Choose one of the criteria as the objective function $z$.

b) Define constraints for the remaining criteria.

c) Optimize the objective function $z=z\left(x_{1}, \ldots, x_{n}\right)$ under the given constraints.

A simple example will immediately clarify how the OR Optimization Principle is to be used. Suppose we have to design a system $S$ and we want to optimize it according to the OR Optimization Principle. We assume that we have the following three criteria

i) $\mathrm{A}=$ availability,

ii) $V=$ volume,

iii) $\mathrm{C}=\cos t$

and $n$ system variables $x_{1}, x_{2}, \ldots, x_{n}$. According to point (a) of the optimization principle, we have to choose one criterion as the objective function. We take, for instance, the availability as the function $z$. For the criteria volume $V$ and cost $C$ we have to define constraints. Suppose we want to make the system $S$ not more voluminous than a volume $V_{0}$ and not more expensive than an amount $C_{0}$. The OR Optimization Principle then finally leads us to the following optimization problem.

Find $\left(x_{1}, \ldots, x_{n}\right)$ such that the objective function $z=A\left(x_{1}, \ldots, x_{n}\right)$ is maximal under the constraints

$$
\begin{aligned}
& V\left(x_{1}, \ldots, x_{n}\right)-V_{0} \leq 0, \\
& C\left(x_{1}, \ldots, x_{n}\right)-C_{0} \leq 0 .
\end{aligned}
$$

Since in most of the optimization problems the system variables can only take values which are greater than or equal to zero, we have, in addition to the constraints (III.1) and (III.2), the set of constraints

$$
x_{i} \geq 0, \quad i=1, \ldots, n,
$$

which are often called the non-negativity conditions of the optimization problem.

The formulation of the optimization problem which we have given for a simple example suggests the following general mathematical formulation of an OR optimization problem.

\section{OR optimization problem}

Given $n$ variables $x_{1}, x_{2}, \ldots, x_{n}$, an objective function $z=z\left(x_{1}, \ldots, x_{n}\right)$, and $m$ constraint functions $\phi_{i}\left(x_{1}, \ldots, x_{n}\right), i=1,2, \ldots, m$. Find $\left(x_{1}, \ldots, x_{n}\right)$ such that $z=z\left(x_{1}, \ldots, x_{n}\right)$ is maximal under the constraints

$$
\phi_{i}\left(x_{1}, \ldots, x_{n}\right) \leq 0, \quad i=1, \ldots, m .
$$


In the formulation of the optimization problem we may always assume that we want to maximize the objective function. For, in the case where we want to minimize it (e.g. if $z=\cos t$ ) we redefine the objective function by taking its negative value (e.g. $z=-\cos t$ ) and we then maximize the new objective function. This procedure does not affect the solution $\left(x_{1}, \ldots, x_{n}\right)$ of the problem. In addition, the constraints, like (III.1), (III.2), and (III.3), can always be written in the form as in (III.4).

We may therefore say if we have a problem of optimal system design to which the OR Optimization Principle can be applied (quantifiable criteria) that its corresponding mathematical problem can always be formulated in the form of the OR Optimization Problem given above. Which criterion we choose as the objective function $z$ and what the constraints on the remaining criteria will be are decisions which have to be made separately in each particular case. We shall learn more about it in the examples which we will discuss later.

Depending on the type of the objective function $z$ and the constraint functions $\phi_{i}$, the OR optimization problems are generally classified as problems of Linear Programming, Nonlinear Programming, etc. For instance, in the case where

$$
\begin{aligned}
z & =\sum_{j=1}^{n} c_{j} x_{j}, \\
\phi_{i} & =\sum_{j=1}^{n} a_{i j} x_{j}, \quad i=1, \ldots, m
\end{aligned}
$$

with given constants $c_{j}, a_{i j}$, for $j=1, \ldots, n$ and $i=1, \ldots, m$, we have a linear programming problem. For such problems there exists a very powerful method of solution, the so-called simplex method. Many practical optimization problems lead to linear programming problems. Unfortunately, reliability optimization problems practically always end up with non-linear problems. For the non-linear problems, however, no general method of solution like the simplex method exists. The reader who is interested in the mathematical methods for the solution of OR optimization problems is referred to the special OR literature ${ }^{15,16)}$.

\section{REDUNDANCY OPTIMIZATION}

In this section we want to discuss a simple example of redundancy optimization. We assume that we have an unrepairable system $\mathrm{S}_{0}$ consisting of two different components $\mathrm{C}_{1}$ and

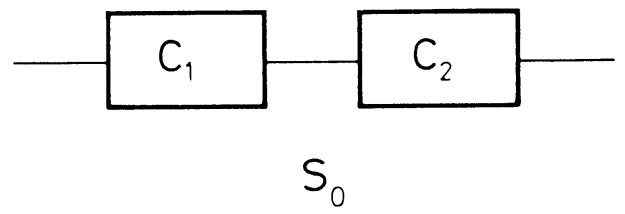

$\mathrm{C}_{2}$ in series connection. To improve the reliability of the system $\mathrm{S}_{0}$ we want to add redundancies in the following way. We add $x_{1}$ hot redundant components of the type $\mathrm{C}_{1}$ to the component $C_{1}$ and $x_{2}$ cold redundant components of the type $C_{2}$ to the component $C_{2} \cdot$. This way we change the system $S_{0}$ to the system $S$ shown in Fig. 25. We then want to find the optimal values for $x_{1}$ and $x_{2}$ under the following assumptions. 


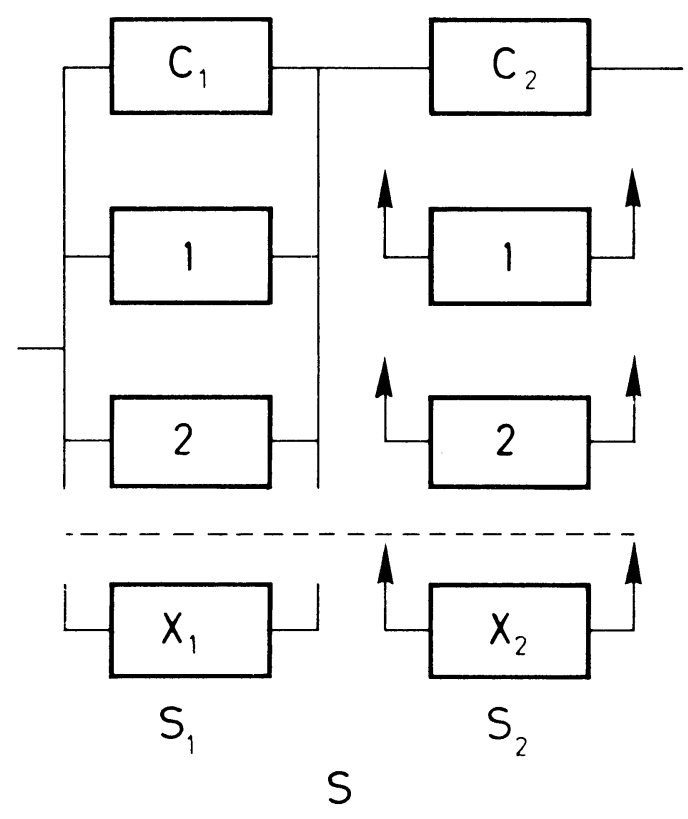

Fig. 25 Improvement of the system $\mathrm{S}_{0}$

a) The lifetimes of $\mathrm{C}_{1}$ and $\mathrm{C}_{2}$ are independent and exponentially distributed.

b) $C_{i}$ has weight $w_{i}$ and $\operatorname{cost} c_{i}, i=1,2$.

c) The system variables are $x_{1}, x_{2}$.

d) The criteria are:

- reliability for a given operation time $t_{0}$,

- weight,

- cost.

e) The objective function $z=$ reliability of $S$.

f) The constraints are:

- weight of redundant components $\leq \mathrm{w}_{0}$,

- cost of redundant components $\leq c_{0}$.

Suppose the subsystems $S_{1}$ and $S_{2}$ of the system $S$ are given as indicated in Fig. 25; we then obtain for the objective function

$$
z=R_{S_{1}}\left(x_{1}\right) R_{S_{2}}\left(x_{2}\right) \text {, }
$$

where

$$
\begin{aligned}
& \mathrm{R}_{\mathrm{S}_{1}}\left(\mathrm{x}_{1}\right)=1-\left(1-\mathrm{e}^{\left.-\lambda_{1} \mathrm{t}_{0}\right)^{\mathrm{x}_{1}+1}},\right. \\
& \mathrm{R}_{\mathrm{S}_{2}}\left(\mathrm{x}_{2}\right)=\mathrm{e}^{-\lambda_{2} \mathrm{t}_{0}} \sum_{j=0}^{\mathrm{x}_{2}} \frac{\left(\lambda_{2} \mathrm{t}_{0}\right)^{j}}{j !} .
\end{aligned}
$$


Equation (III.7) is a consequence of the independence of the lifetimes of $C_{1}$ and $C_{2}$. Formula (III.8) follows from Theorem 4 and the fact that $C_{1}$ has an exponential lifetime distribution with failure rate $\lambda_{1}$. Formula (III.8) follows analogously from Theorem 7, Eq. (I.34).

The analytic expressions for the weight and cost of the redundant components of the system $\mathrm{S}$ are obviously given by

$$
\begin{aligned}
& \mathrm{w}=\mathrm{w}_{1} \mathrm{x}_{1}+\mathrm{w}_{2} \mathrm{x}_{2}, \\
& \mathrm{c}=\mathrm{c}_{1} \mathrm{x}_{1}+\mathrm{c}_{2} \mathrm{x}_{2} .
\end{aligned}
$$

We can therefore formulate our redundancy optimization problem in the following:

Find $\left(\mathrm{x}_{1}, \mathrm{x}_{2}\right)$ such that

$$
z=R_{S_{1}}\left(x_{1}\right) R_{S_{2}}\left(x_{2}\right)
$$

is maximal under the constraints

$$
\begin{aligned}
& \mathrm{w}_{1} \mathrm{x}_{1}+\mathrm{w}_{2} \mathrm{x}_{2} \leq \mathrm{w}_{0}, \\
& \mathrm{c}_{1} \mathrm{x}_{1}+\mathrm{c}_{2} \mathrm{x}_{2} \leq \mathrm{c}_{0}, \\
& \mathrm{x}_{1}, \mathrm{x}_{2} \geq 0 .
\end{aligned}
$$

We see that in this problem the constraints are linear, but the objective function is non-1inear.

We want to discuss a numerical example for this problem. We take

$$
\begin{array}{lll}
\lambda_{1}=5 \times 10^{-4}, & \lambda_{2}=8 \times 10^{-4}, & \mathrm{t}_{0}=1000 \mathrm{~h}, \\
\mathrm{c}_{0}=\$ 180, & \mathrm{c}_{1}=\$ 30, & \mathrm{c}_{2}=\$ 10, \\
\mathrm{w}_{0}=9 \mathrm{~kg}, & \mathrm{w}_{1}=1 \mathrm{~kg}, & \mathrm{w}_{2}=1 \mathrm{~kg},
\end{array}
$$

The constraints (III.13) and (III.14) then become

$$
\begin{array}{r}
x_{1}+x_{2} \leq 9, \\
30 x_{1}+10 x_{2} \leq 180 .
\end{array}
$$

These inequalities together with expression (III.15) define the so-called feasible solutions to our optimization problem, which are all the integer-valued combinations $\left(x_{1}, x_{2}\right)$ which satisfy the inequalities (III.15) to (III.17). Figure 26 shows the region of the feasible solutions given by the shaded area in the first quadrant of the $\left(x_{1}, x_{2}\right)$ plane.

To find the optimum solution $\left(x_{1}, x_{2}\right)$ to our problem, we only have to calculate the right-hand side of Eq. (III.12), given by Eqs. (III.8) and (III.9) for all feasible solutions and the solution of our problem will be the point $\left(x_{1}, x_{2}\right)$ which yields the largest value for the objective function $z$. It is, however, not necessary to do the calculation for every feasible solution because of the following fact. Suppose we keep $x_{2}$ fixed, then $z=z\left(x_{1}, x_{2}\right)$ is a monotonically increasing function of $x_{1}$ as is easily seen from Eqs. (III.12) and (III.8). 


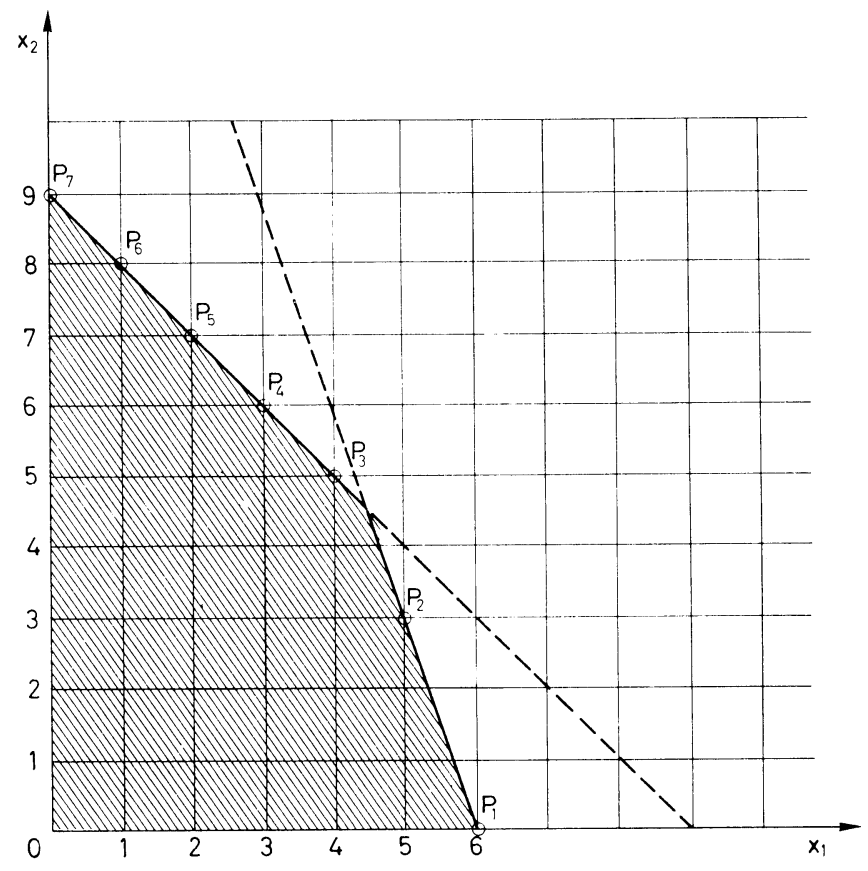

Fig. 26 Feasible solutions

Therefore, for fixed $x_{2}$, the feasible solution $\left(x_{1}, x_{2}\right)$ with the highest possible $x_{1}$ will yield the highest possible z-value for fixed $x_{2}$. An analogous effect can be seen by keeping $x_{1}$ fixed and varying $x_{2}$ within the region of feasible solutions. Doing that one easily finds that the solution to our optimization problem must be among the points $P_{1}$ to $P_{7}$ shown in Fig. 26. A numerical evaluation of the objective function at these points yields:

$$
\begin{aligned}
& z\left(P_{1}\right)=0.449, \quad z\left(P_{2}\right)=0.987, \quad z\left(P_{3}\right)=0.990, \\
& z\left(P_{4}\right)=0.976, \quad z\left(P_{5}\right)=0.939, \quad z\left(P_{6}\right)=0.845 \text {, } \\
& z\left(P_{7}\right)=0.607 \text {. }
\end{aligned}
$$

This shows that the point $\mathrm{P}_{3}$ with

$$
\mathrm{x}_{1}=4, \quad \mathrm{x}_{2}=5
$$

yields the solution. The corresponding values of the criteria are:

$$
\text { reliability }=0.990, \quad \text { weight }=9 \mathrm{~kg}, \quad \text { cost }=\$ 170 .
$$

So far we have chosen the reliability of the system $S$ as the objective function. We now modify the problem and take the cost as the objective function. For the reliability we take the constraint that the reliability of the system should be at least 0.95 . We then have the following optimization problem.

Find $\left(x_{1}, x_{2}\right)$ such that

$$
z=30 x_{1}+10 x_{2}
$$


is minimal under the constraints

$$
\begin{aligned}
x_{1}+x_{2} & \leq 9, \\
R_{S_{1}}\left(x_{1}\right) R_{S_{2}}\left(x_{2}\right) & \geq 0.95, \\
x_{1}, x_{2} & \geq 0 .
\end{aligned}
$$

In this case one can proceed in an analogous way to the previous problem. It is, however, slightly more difficult to find the feasible solutions. The reader may try it as an exercise. The solution to the problem is given by

$$
x_{1}=x_{2}=3 \text {. }
$$

The corresponding values of the criteria are:

$$
\text { reliability }=0.967, \text { weight }=6 \mathrm{~kg}, \quad \text { cost }=\$ 120 .
$$

In the first problem we have put priority on the criterion "reliability". In the second problem we have put priority on the criterion "cost". Which one of the solutions we will use later for the actual design depends on other factors. We may, for instance, say that $96.7 \%$ reliability instead of $99 \%$ is enough for us, but we then have the advantage of having a system which is $33 \%$ lighter and $34 \%$ cheaper. In any case, we observe that it may be worth while to formulate the original problem in different ways to have the possibility of looking at different possible solutions to the original problem.

\section{NUMBER OF SPARE PARTS}

\subsection{Average number of spare parts}

Before we come to the discussion of an example to determine the optimum number of spare parts for the components of a given system, we first want to give a very simple and useful formula to determine the average number of spare parts for components of a system. We make the following assumptions:

a) $\mathrm{n}$ unrepairable components of the same type, having reliability $\mathrm{R}(\mathrm{t})$, operate under the same conditions in a system $\mathrm{S}$.

b) The system $\mathrm{S}$ fails if one of these components fails.

On the basis of these assumptions, we ask the question: "What is the average number $\bar{n}$ of spare parts we should provide for these $n$ components during the time $[0, t]$ ?" The answer is

$$
\overline{\mathrm{n}}=\mathrm{n}[1-\mathrm{R}(\mathrm{t})]
$$

To prove this simple, but very helpful, formula we define $\mathrm{n}$ random variables

$$
N_{i}= \begin{cases}1, & \text { if component } C_{i} \text { fails during }[0, t], \\ 0, & \text { otherwise . }\end{cases}
$$


for $i=1,2, \ldots, n$. We obviously have

$$
\begin{aligned}
& P\left(N_{i}=1\right)=1-R(t), \\
& P\left(N_{i}=0\right)=R(t),
\end{aligned}
$$

for $i=1,2, \ldots, n$.

The number $\mathrm{N}$ of failed components during $[0, t]$ is given by

$$
N=\sum_{i=1}^{n} N_{i},
$$

and its expected value is obtained by

$$
\begin{aligned}
E[N] & =E\left[\sum_{i=1}^{n} N_{i}\right]=\sum_{i=1}^{n} E\left[N_{i}\right] \\
& =\sum_{i=1}^{n}\{0 \cdot R(t)+1 \cdot[1-R(t)]\}=n[1-R(t)] .
\end{aligned}
$$

The average number $\bar{n}$ of spare parts should, however, be equal to the average number of failed components which is given by formula (III.21) and is identical to Eq. (III.18).

The formula (III.18) can easily be applied in practice, if $R(t)$ is known. Since normally the time $t=t_{0}$ to which the formula is to be applied will be fixed, we only have to provide an estimate for the value $R\left(t_{0}\right)$ and not for the whole function $R(t)$. This, however, can easily be done in many cases by using failure data of those components.

\subsection{Optimum number of spare parts for a series connection}

Now we come to an optimization problem in connection with the determination of the number of spare parts of a system. We discuss the following example.

We consider a series connection $S$ of

$$
\begin{aligned}
& m_{1} \text { unrepairable components of type } C_{1} \text {, } \\
& m_{2} \text { unrepairable components of type } C_{2} \text {. }
\end{aligned}
$$

We want to find the optimum numbers $\left(x_{1}, x_{2}\right)$ of spare parts for $C_{1}, C_{2}$ using the following assumptions:

a) $C_{1}, C_{2}$ have independent 1 ifetimes and constant failure rates $\lambda_{1}$ and $\lambda_{2}$, respectively.

b) Cost of $\mathrm{C}_{i}$ is $c_{i}, i=1,2$.

c) New spare parts of type $C_{i}$ arrive at times $t_{i}, 2 t_{i}, 3 t_{i}, \ldots, i=1,2$. .

d) The system variables are:

$$
x_{i}=\text { number of spare parts for } C_{i}, i=1,2 .
$$

e) The criteria are:

- Permanent availability = probability that the system $S$ is never down because of a lack of spare parts.

- Cost of spare parts.

- Storage place for spare parts. 
f) The objective function is $z=$ permanent availability.

g) The constraints are

$$
\begin{gathered}
c_{1} x_{1}+c_{2} x_{2} \leq c_{0}, \\
0 \leq x_{1} \leq k_{1}, \\
0 \leq x_{2} \leq k_{2},
\end{gathered}
$$

where $k_{1}$ and $k_{2}$ express the limitations in storage places for spare parts of type $C_{1}$ and $\mathrm{C}_{2}$, respectively, and $\mathrm{c}_{0}$ is the constraint on cost.

Suppose we denote by $N_{i}(t)$ the number of components of type $C_{i}$ which failed during $[0, t]$. From Theorem 7 , formula (1.34), it then easily follows that

$$
P\left(N_{i}\left(t_{i}\right) \leq x_{i}\right)=e^{-m_{i} \lambda_{i} t_{i}} \sum_{j=0}^{x_{i}} \frac{\left(m_{i} \lambda_{i} t_{i}\right)^{j}}{j !}, \quad i=1,2 .
$$

In addition, since our system $\mathrm{S}$ is a series connection of independent components of type $C_{1}$ and $C_{2}$, it is easily seen that the objective function which is supposed to be equal to the permanent availability of $\mathrm{S}$, defined under (e) above, is given by

$$
z=P\left(N_{1}\left(t_{1}\right) \leq x_{1}\right) P\left(N_{2}\left(t_{2}\right) \leq x_{2}\right),
$$

where the right-hand side of Eq. (III.26) is determined by the formula (III.25).

The rigorous formulation of our optimization problem is now:

Find $\left(\mathrm{x}_{1}, \mathrm{x}_{2}\right)$ such that the objective function (III.26) is maximal under the constraints (III.22) to (III.24).

As a numerical example of this problem we take:

$$
\begin{aligned}
& \lambda_{1}=1.5 \times 10^{-5}, \quad \lambda_{2}=10^{-4}, \\
& \mathrm{t}_{1}=\mathrm{t}_{2}=1000 \mathrm{~h} \approx 42 \text { days }, \\
& \mathrm{m}_{1}=200 \text { units, } \mathrm{m}_{2}=50 \text { units, } \\
& \mathrm{k}_{1}=12 \text { units, } \quad \mathrm{k}_{2}=8 \text { units }, \\
& \mathrm{c}_{0}=\$ 60, \quad \mathrm{c}_{1}=\$ 4, \quad \mathrm{c}_{2}=\$ 3 .
\end{aligned}
$$

Putting these values in the formulae (III.22) to (III.25), one finds analogously to the problems considered in Section 3 that the solution to this problem is given by

$$
x_{1}=9, \quad x_{2}=8
$$

The corresponding values of the criteria are:

$$
\begin{aligned}
\text { permanent availability } & =0.931, \\
\text { cost of spare parts } & =\$ 60 .
\end{aligned}
$$


We now modify the problem such that we set $k_{2}=\infty$ in the constraint (III.24). That means, there is now storage limitation for spare parts of type $C_{2}$. The solution to the modified problem again can be found in the same way as before, and it is given by

$$
x_{1}=7, \quad x_{2}=10
$$

The corresponding values for the criteria are:

$$
\begin{aligned}
\text { permanent availability } & =0.975, \\
\text { cost of spare parts } & =\$ 58 .
\end{aligned}
$$

Let us now compare both solutions. In each case we have $17=9+8=7+10$ spare parts all together. In the second case we have a higher availability and lower cost. Of course, in the second case we have two spare parts of type $\mathrm{C}_{2}$ more than the storage limitations in the first problem formulation allow. On the other hand, we have only seven spare parts of type $C_{1}$ where we could have $k_{1}=12$. Suppose we are able to put the two spare parts of type $\mathrm{C}_{2}$ on the five empty places of type $\mathrm{C}_{1}$ parts, the second formulation of the problem then really led us to a solution of which we did not think in the first formulation of the problem and which is even more advantageous for us than the first one when we compare the values (III.28) and (III.29) with (III.31) and (III.32).

This simple example again shows how useful different formulations of a general optimization problem can be.

\section{OPTIMUM TIME INTERVAL FOR AGE REPLACEMENT}

As a last example of optimization problems in connection with reliability we want to discuss how to find the optimum time interval for an age replacement policy. We generally call a replacement policy a rule which says when a component has to be replaced by a new one. Here, we consider the so-called age replacement policy. It says that a component is replaced:

a) if it failed, or

B) after $x$ time units.

Optimization of the time interval for age replacement now means: Find the value of $x$ which is optimal with respect to a given criterion.

Before we formulate our problem in a rigorous form, we make the following assumptions:

a) The system in which the considered component operates has to run for a long time $(t \rightarrow \infty)$.

b) The failure distribution function $F(t)$ of the component is known.

c) $c_{1}=$ average cost to replace a failed component,

$c_{2}=$ average cost to replace a non-failed component,

$c_{2}<c_{1}$.

We now use the following notation:

$N_{1}(t)=$ number of failed components during $[0, t]$.

$\mathrm{N}_{2}(t)=$ number of exchanges of non-failed components during $[0, t]$. 
The average cost per time unit for age replacement during $[0, t]$ is then given by

$$
C(t ; x)=\frac{c_{1} E\left[N_{1}(t)\right]+c_{2} E\left[N_{2}(t)\right]}{t}
$$

According to our assumption (a) on page 60, we want to consider $t \rightarrow \infty$. We therefore formulate our optimization problem in the following way:

Find the replacement time interval $x$ such that the average cost per time unit for $t \rightarrow \infty$

$$
z(x)=\lim _{t \rightarrow \infty} C(t ; x)
$$

is minimal.

We could also use the function (III.33) as the objective function for given $t$. In this case the solution $x$ will be dependent on $t$ and it turns out that this problem is much more difficult. Its solution is discussed in Ref. 6.

Let us now solve the above formulated problem. In Ref. 6 it is shown that the function $z(x)$, defined in Eq. (III.34) is given by

$$
z(x)=\frac{\left(c_{1}-c_{2}\right) F(x)+c_{2}}{\int_{0}^{x}[1-F(\xi)] d \xi},
$$

where $F(x), c_{1}$ and $c_{2}$ are defined in the above-mentioned assumptions (b) and (c).

The function (III.35) takes its minimum at the root $x_{0}$ of the equation

$$
\lambda(x) \int_{0}^{x} R(\xi) d \xi-F(x)=\frac{c_{2}}{c_{1}-c_{2}},
$$

where

$$
\begin{aligned}
& \lambda(x)=\frac{F^{\prime}(x)}{R(x)}, \\
& R(x)=1-F(x)
\end{aligned}
$$

are the failure rate and the reliability, respectively, of the component. Equation (III.36) is obtained by differentiating the function (III.35) and setting the derivative equal to zero

Suppose $x_{0}$ is a root of Eq. (III.36), then the function $z(x)$, given by Eq. (III.35), takes the value

$$
z\left(x_{0}\right)=\left(c_{1}-c_{2}\right) \lambda\left(x_{0}\right)
$$

Let us consider two examples.

Example 1: Suppose the component has an exponential failure distribution function

$$
F(x)=1-e^{-\lambda x}
$$


In this case the left-hand side of Eq. (III.36) yields

$$
\lambda \int_{0}^{x} e^{-\lambda \xi} d \xi-\left(1-e^{-\lambda x}\right)=1-e^{-\lambda x}-\left(1-e^{-\lambda x}\right)=0
$$

Since the right-hand side of Eq. (III.36) is never equal to zero, that equation has no solution in this case. The function $z(x)$ therefore has no minimum for finite $x$. This fact suggests making replacements only at failure if a component has constant failure rate, a result which we expect if we take into account that a component with constant failure rate does not age (Chapter I, pages 9 and 10).

Example 2: Suppose the component has a Weibull failure distribution with

$$
\mathrm{F}(\mathrm{x})=1-\mathrm{e}^{-\lambda \mathrm{x} \alpha}, \quad \alpha>1
$$

In this case, as we know from Chapter I, the component has an IFR failure law (increasing failure rate) and we expect a finite $\mathrm{x}$ as the optimum interval for age replacement.

As a numerical example we take

$$
\alpha=2, \quad \lambda=10^{-4}, \quad c_{1}=\$ 1100, \quad c_{2}=\$ 100
$$

We then easily find from Eqs. (III.36), (III.37), and (III.38):

$$
\begin{aligned}
x_{0} & =32 h, \\
z\left(x_{0}\right) & =\$ 6.4 / h .
\end{aligned}
$$

We want to compare the minimum cost per hour for age replacement given by Eq. (III.39) with the cost per hour for a replacement only at failure. In Ref. 6 it is shown that this cost per hour is generally given by

$$
\mathrm{C}_{0}=\frac{\mathrm{C}_{1}}{\mathrm{MTTF}}
$$

For the Weibull distribution with $\alpha=2$ it is easily found that

$$
\mathrm{NTTF}=\frac{1}{2} \sqrt{\frac{\pi}{\lambda}}
$$

The formula (III.40) therefore leads in our case to

$$
\mathrm{C}_{0}=\$ 12.4 / \mathrm{h}
$$

Comparing Eq. (III.39) with Eq. (III.42) shows that age replacement leads to a $48^{\circ}$ cost reduction in this case.

\section{Acknowledgements}

The author wishes to thank Dr. C.S. Taylor for the encouragement to give this lecture series at CERN and Mr. K.S. Kölbig for his helpful criticism in reading the manuscript. 


\section{BASIC CONCEPTS IN PROBABILITY THEORY AND STATISTICS}

\section{INTRODUCTION}

Probability theory is occupied with mathematical models which are first of all used in statistics. There are several concepts forming the basis of probability theory. It seems to be more favourable to discuss first the intuitive meaning of these concepts before giving their rigorous mathematical definition. This is even more important here, since we will give no proofs of the later formulated theorems.

The most important concepts which we shall define in the following sections are:

Random experiment

Sample space

Event

Probability of an event

Probability space

Random variable

Probability law

Distribution function.

We shall discuss these concepts successively, to obtain an idea why they are defined in the way we do in the following sections.

Let us consider the goal of statistics. In principle, one wants to say "something" about the outcome of such experiments, which can have a different outcome even if the experiments are performed under the same conditions.

What is now this "something" that one wants to say about the results of such experiments? It is impossible to predict the result itself of such an experiment before having performed it. But it is perhaps possible to predict "something" about the chances which the different possible outcomes of the experiment have of being observed. This is exactly what statistics does. It tries to measure the chances which different possible results of such experiments have of being observed. The corresponding measure is called the probability p.

But how do we come to a rigorous definition of this measure?

If such an experiment (e.g. tossing a coin) is performed very often under the same conditions one observes that the relative frequency of any particular result stabilizes around a value $\mathrm{p}$ with an increasing number of repetitions of the experiment. In other words, one can imagine that the relative frequency tends to a limit $\mathrm{p}$ when the experiment is performed indefinitely. It is this ideal value $\mathrm{p}$ which intuitively we would like to take as the probability p. However, in reality this value can never be realized. Therefore, in probability theory the existence of such a limit value is demanded axiomatically and one then shows that the relative frequency tends to $\mathrm{p}$. We have seen that the limit value of the relative frequency can only be reached if the experiment is performed indefinitely. This implies that the experiment must have the property of being able to be 
performed indefinitely, at least in principle. This leads us to the concept of a random experiment. It is an experiment which, when performed under the same conditions, can have different outcomes and which can be performed indefinitely, at least in principle.

Examples of a random experiment are:

a) Tossing a coin.

b) Counting the number of failures of a large set of identical electronic equipment.

c) Measuring the lifetime of transistors.

d) Measuring the three velocity components of a turbulent flow at a certain point.

Let us consider a random experiment $\delta$. We then call the set of all its possible different outcomes the sample space $\Omega$ of the random experiment $\delta$.

In the example (a) $\Omega$ has only two elements: $\Omega=\{$ head, tail $\}$. Here, $\Omega$ is finite. In all the other examples $\Omega$ is (at least theoretically) infinite. But also from example (a) it is possible to construct an experiment $\delta_{1}$ which has an infinite sample space $\Omega_{1}$. This is simply done by repeating the original experiment $\delta$ in (a) indefinitely. The elements of $\Omega_{1}$ are then infinite sequences of the two elements $\{$ head\} or $\{$ tail\}.

In the applications one is often not so much interested in the different elements (results of the experiment) of the sample space $\Omega$, as in certain groups of elements. In example (b) it may be desirable to know what the probability is that "at least 10 failures" are observed or that "at most 20 failures" occur. That means, one is interested in the probability of certain subsets of the sample space $\Omega$. Such subsets of $\Omega$ are called events A, B, ... of the random experiment 8 . The probability of an event A is (intuitively) simply the limit of the relative frequency of this event where we say that the event is observed whenever an element in $\mathrm{A}$ is observed.

We have seen that an event is a set and that the probability is a function of such sets, i.e. the probability is a set function. It assigns to a subset (event) of the sample space $\Omega$ a value $\mathrm{p}: 0 \leq \mathrm{p} \leq 1$, the probability of the corresponding event.

In principle, one could define that every subset of the sample space represents an event. But it turns out that in this case there arise mathematical difficulties. Therefore one considers only special classes of subsets of $\Omega$ to represent events. (Experience shows that this kind of event is sufficient for the applications.) The classes of subsets which are taken as events are $\sigma$ algebras. A $\sigma$ algebra $t$ of subsets of $\Omega$ is defined to be a class of subsets of $\Omega$ which contains the space $\Omega$, the nul set $\phi$ and with each $A \in t$ (A element of $t$ ), its complement $\bar{A} \in t$. In addition, the operation of union is countably often permitted. This means, if $A_{i} \in t, i=1,2, \ldots$, the union

$$
\bigcup_{i=1}^{\infty} A_{i}
$$

is also an element of $t$, i.e. it is also an event.

Let us now briefly summarize. We consider a random experiment $\varepsilon$ with the sample space $\Omega$. We suppose that we have selected what we consider to be the events of this experiment. In other words that we have assigned to $\&$ a $\sigma$ algebra $t$. (We shall see later how to do this in practical cases.) Suppose that for each event $A \in t$ we also know the 
probability $\mathrm{p}(\mathrm{A})$. We then have assigned to the random experiment the triplet $(\Omega, t, \mathrm{p})$, which represents a mathematical model of the random experiment $\delta$. The triplet $(\Omega, t, p)$ is called a probability space.

For the applications this model is too general and not very handy. Therefore one introduces standardized sample spaces. For one-dimensional problems $\Omega=\mathrm{R}$ (= real axis) is taken and for $n$-dimensional problems $\Omega=R_{n}(=n$-dimensional real space). The models for the events $t$ are also standardized by taking what are called the Borel sets $\mathcal{B}_{n}$ in $R$ or $R_{n}$. Without going into mathematical details we mention that, for instance, points in $R_{n}$, intervals in $\mathrm{R}$, rectangles in $\mathrm{R}_{2}$, etc., are Borel sets. Therefore practically all mathematical models for random experiments, which are used in the applications, are of the form $\left(R_{n}, \mathscr{B}_{n}, P\right)$, where $\mathrm{n}$ is properly chosen as we shall see later, and where $\mathrm{P}$ describes the probability of each event $B_{n} \in \mathscr{B}_{n}$.

But how can we construct this model if originally $\Omega$ is not equal to $R_{n}$ for some $n$ ? This is conceptually very simple. We take a transformation $\underline{x}=\left(x_{1}, x_{2}, \ldots, x_{n}\right)$ from $\Omega$ into $R_{n}$. [For instance, in our example (a) we take $n=1$ and assign to the element \{head\} the number $X_{1}=0$ and to $\{$ tail $\}$ the number $\left.x_{1}=1.\right]$ For reasons which we shall understand immediately, the transformation must have the following property:

Let $B_{n} \in \mathscr{B}_{n}$ be an event (= Borel set) in $R_{n}$. Then the inverse image

$$
\begin{aligned}
A=\underline{X}^{-1}\left(B_{n}\right)= & \{\text { all elements } \omega \text { in } \Omega, \text { for which the value } \underline{X}(\omega) \\
& \text { lies in the set } \left.B_{n}\right\}
\end{aligned}
$$

of the transformation $\underline{X}$ must be an element in t. In other words, events (in $\Omega$ ) must be transformed into events (in $R_{n}$ ).

With this property of the transformation we can easily define in a consistent way the probability of $B_{n}$ :

$$
P\left(B_{n}\right)=p(A)
$$

The transformation $\underline{x}$ is called an $\underline{n-d i m e n s i o n a l ~ r a n d o m ~ v a r i a b l e . ~(I f ~} \underline{x}$ is one-dimensional, we write $\left.X_{.}\right)$The components $X_{i}$ of $\underline{X}$ are all one-dimensional random variables. The set function $P\left(B_{n}\right)$ is called the probability law or the distribution of $\underline{x}$. In general, we shall denote random variables by capital letters and particular values which they take by sma11 letters.

We have seen that $P\left(B_{n}\right)$ is a set function. It is much more convenient and practical to deal with functions which are only dependent on points $\underline{x} \in R_{n}$ (point functions). On the other hand, it can be shown that for each distribution $P\left(B_{n}\right)$ there exists a (point) function $\mathrm{F}(\underline{\mathrm{x}})$ such that

$$
P\left(B_{n}\right)=\int_{B_{n}} d F(\underline{x})
$$

for each $B_{n} \in \mathscr{B}_{n}$. Therefore, we can always express the distribution of $\underline{x}$ with the aid of the function $F(\underline{x})$. The function $F(\underline{x})$ is called the distribution function of the random variable $\underline{x}$. 
Finally let us briefly discuss the meaning and the application of the probability spaces $\left(R_{n}, \mathscr{H}_{n}, P\right)$ in statistics. In a practical problem, we have, in principle, the following steps:

i) We have a random experiment $\delta$.

ii) $\varepsilon$ determines, mostly in a natural way, the sample space $\Omega$ and the events $t$. [In the examples (a) to (d) we have:

a) $\Omega=\{$ head, tail $\} ; t=\operatorname{set} \mathscr{T}(t)$ of all subsets of $\Omega$

b) $\Omega=\{0,1,2, \ldots\}$; $t=T(t)$

c) $\Omega=(0, \infty) ; \quad t=(0, \infty) \cap \mathcal{B}_{1}$

d) $\left.\Omega=R_{3}, \quad t=\mathfrak{B}_{3} \cdot\right]$

iii) We try to find a random variable $\underline{X}$ which corresponds to the random experiment $\mathcal{E}$. This also is mostly done in a natural way. [In our examples we have:

a) $X($ head $)=x_{1}, \quad X($ tail $)=x_{2}, \quad x_{1} \neq x_{2}, \quad$ e.g. $x_{1}=0, \quad x_{2}=1$.

b) $X(\omega)=\omega, \quad \omega=0,1,2, \ldots$

c) $X(\omega)=\omega, \quad 0<\omega<\infty$

d) $\left.\underline{X}(\underline{\omega})=\underline{\omega}, \underline{\omega} \in R_{3} \cdot\right]$

iv) We must find the distribution of $\underline{x}$; in other words, we must find the distribution function $F(\underline{x})$ which corresponds to the random experiment $\delta$.

In other words, knowing what the random variable $\underline{X}$ corresponding to our random experiment is (dimension $\mathrm{n}$ and domain) and knowing its distribution function $\mathrm{F}(\underline{\mathrm{x}})$ we have the complete mathematical description of our random experiment. That means, for each event represented by a Borel set $B_{n}$ we can compute its probability by the formula (A.1) .

As far as statistics is concerned it is now step (iv) where statistics normally is used. For, probability theory only tells us that there exists a distribution function $F(\underline{x})$, but it does not tell us how to find it in a particular case. Statistics exploits information about $F(\underline{x})$ coming from a finite number of observations of $\underline{x}$, called samples from $\underline{x}$. It provides methods to find approximate values for the distribution function using the sampled values.

For instance, in our example (a) we know from probability theory that

$$
\mathrm{P}\left(\mathrm{x}_{1}\right)=\mathrm{p}, \quad \mathrm{P}\left(\mathrm{x}_{2}\right)=1-\mathrm{p}, \quad 0<\mathrm{p}<1
$$

For a particular coin we have to determine $\mathrm{p}$. We toss the coin $\mathrm{n}$ times and take the observed relative frequency of $x_{1}$ as an approximate value of $p$.

There are of course cases where we have additional information about the random experiment by which we can determine the distribution of the corresponding random variable without taking samples.

Suppose we know in our example (a) that the coin is honest. We can then assume that $P\left(x_{1}\right)=P\left(x_{2}\right)$, which leads to $p=1-p$ or $p=1 / 2$.

Summarizing we can say that probability theory studies the properties of mathematical models (= probability spaces) of random experiments and especially the properties of distribution functions. Statistics, however, studies and provides methods to determine unknown distributions using information drawn from samples of the corresponding random variables. 


\section{DEFINITIONS OF THE FUNDAMENTAL CONCEPTS}

We do not give the rigorous mathematical definition of all the concepts which we have discussed above. In fact, this is not necessary for our purpose.

We know that an event is a subset of the sample space $\Omega$. In probability theory it is common to use the following correspondences. We denote by $A_{1}$ and $A_{2}$ two events, and by the same notations the subsets in $\Omega$ which correspond to them. We then have the correspondences:

\section{Events}

$A_{1}$ occurs

$A_{1}$ does not occur

$A_{1}$ or $A_{2}$ occurs

$A_{1}$ and $A_{2}$ occur

$A_{1}$ implies $A_{2}$

$\phi$ impossible event

$\Omega$ sure event

$A_{1}$ and $A_{2}$ are equivalent

$A_{1}$ and $A_{2}$ are exclusive

\section{Subsets of $\Omega$}

$$
\begin{aligned}
& \left\{\omega \in A_{1}\right\} \\
& \left\{\omega \in \bar{A}_{1}\right\} \text { (complement) } \\
& A_{1} \cup A_{2} \text { (union) } \\
& A_{1} \cap A_{2} \text { (intersection) } \\
& A_{1} \subset A_{2} \text { (inclusion) } \\
& \phi \text { (empty set) } \\
& \Omega \\
& A_{1}=A_{2} \\
& A_{1} \cap A_{2}=\phi \text { (disjoint). }
\end{aligned}
$$

We know already that the probability $\mathrm{P}$ is a set function which takes values between 0 and 1 on the elements of a $\sigma$ algebra $t$, i.e. on the events. There are other properties which the probability $P$ reasonably should have. They all together lead us to the following:

\section{Probability axioms:}

Let $\Omega$ be a sample space and $\mathcal{t}$ a $\sigma$ algebra of events in $\Omega$. A real set function $\mathrm{P}$, defined on $\mathfrak{t}$, is called a probability on $\mathfrak{t}$, if:

$$
\begin{array}{ll}
\text { I) } P(A) \geq 0 & \text { for all events } A \in t, \\
\text { II) } P\left(\bigcup_{i=1}^{\infty} A_{i}\right)=\sum_{i=1}^{\infty} P\left(A_{i}\right) & \text { with } A_{i} \in \mathcal{A}, \text { and } A_{i} \cap A_{j}=\phi \text { for } i \neq j, \\
\text { III) } P(\Omega)=1 . &
\end{array}
$$

Let us briefly discuss these axioms in order to obtain an idea whether a probability defined in this way is reasonable from an intuitive point of view. To see this we shall always consider the corresponding relative frequencies, which according to what we have said before should be approximations to the axiomatically introduced probabilities.

The first axiom is reasonable since the relative frequencies $Q_{n}(n=$ number of trials) satisfy $Q_{n} \geq 0$ for all $n=1,2, \ldots$.

The last axiom is also reasonable because the relative frequency of the sure event is always 1. In other words, one of the possible outcomes of a random experiment is always observed.

Let us take the second axiom for two exclusive events $A_{1}$ and $A_{2}$. In this case axiom (II) tells us that the probability of the event " $A_{1}$ or $A_{2}$ " is given by the sum of the single probabilities of $A_{1}$ and $A_{2}$. This is also reasonable. For, if we take the relative frequencies $Q_{n 1}$ and $Q_{n 2}$ of $A_{1}$ and $A_{2}$, respectively, the relative frequency $Q_{n}$ of the event " $A_{1}$ or $A_{2}$ " is then given by $Q_{n}=Q_{n_{1}}+Q_{n_{2}}$ for all $n=1,2, \ldots$. 
Let us take another example to test whether the axioms are justified from an intuitive point of view. Since

$$
\Omega \cup \phi=\Omega \quad \text { and } \quad \Omega \cap \phi=\phi \text {, }
$$

it follows from (II) and (III) that

$$
1=\mathrm{P}(\Omega)=\mathrm{P}(\Omega \cup \phi)=\mathrm{P}(\Omega)+\mathrm{P}(\phi)
$$

and from (I) it follows that

$$
\mathrm{P}(\phi)=0,
$$

which is also reasonable. In a similar way, it can be shown that

$$
P(\bar{A})=1-P(A)
$$

for every event $A \in \mathcal{A}$.

We mention two other rules which are very useful and can be proved intuitively using Fig. A.1:

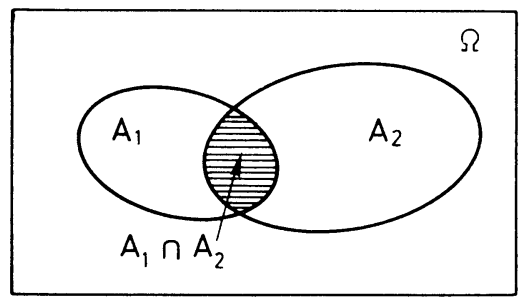

Fig. A.1 Intersection of $A_{1}$ and $A_{2}$

$$
\begin{aligned}
& \mathrm{P}\left(\mathrm{A}_{1} \cup \mathrm{A}_{2}\right)=\mathrm{P}\left(\mathrm{A}_{1}\right)+\mathrm{P}\left(\mathrm{A}_{2}\right)-\mathrm{P}\left(\mathrm{A}_{1} \cap \mathrm{A}_{2}\right), \\
& \mathrm{P}\left(\overline{\mathrm{A}}_{1} \cap \overline{\mathrm{A}}_{2}\right)=1-\mathrm{P}\left(\mathrm{A}_{1} \cup \mathrm{A}_{2}\right),
\end{aligned}
$$

where $A_{1}$ and $A_{2}$ are any two events.

\section{CONDITIONAL PROBABILITY. INDEPENDENCE}

To better understand the concept of conditional probability we start with an example.

Let $\delta$ be a random experiment and $A$ and $B$ two events associated with $\delta$. It is easy to check that

$$
(\mathrm{A} \cap \mathrm{B}) \cup(\overline{\mathrm{A}} \cap \mathrm{B}) \cup(\mathrm{A} \cap \overline{\mathrm{B}}) \cup(\overline{\mathrm{A}} \cap \overline{\mathrm{B}})=\Omega
$$


and that the four events in parentheses are mutually exclusive. We now perform the random experiment $\delta \mathrm{n}$ times in an independent way (i.e. each trial is independent of the outcome of the preceding trials). We suppose that we observed the four events with the frequencies given in the following table:

\begin{tabular}{|c|c|c|}
\cline { 2 - 3 } \multicolumn{1}{c|}{} & $B$ & $\bar{B}$ \\
\hline$A$ & $n_{11}$ & $n_{12}$ \\
\hline $\bar{A}$ & $n_{21}$ & $n_{22}$ \\
\hline
\end{tabular}

We now calculate the relative frequency $Q(A \mid B)$ of $A$ given that $B$ was observed. The result is

$$
\mathrm{Q}(\mathrm{A} \mid \mathrm{B})=\frac{\mathrm{n}_{11}}{\mathrm{n}_{11}+\mathrm{n}_{21}} \text {. }
$$

If $Q(A \cap B)$ gives the relative frequency of the event $(A \cap B)$ and $Q(B)$ the one of $B$, we can write Eq. (A.6) as

$$
\mathrm{Q}(\mathrm{A} \mid \mathrm{B})=\frac{\mathrm{n}_{11} / \mathrm{n}}{\left(\mathrm{n}_{11}+\mathrm{n}_{21}\right) / \mathrm{n}}=\frac{\mathrm{Q}(\mathrm{A} \cap \mathrm{B})}{\mathrm{Q}(\mathrm{B})} .
$$

If we now replace on the right-hand side of Eq. (A.7) the relative frequencies by their corresponding probabilities, we then interpret the term obtained as "the probability of A under the condition that B has occurred". Therefore we give the following definition:

\section{Definition 1}

Let $\mathrm{A}$ and $\mathrm{B}$ be two events with $\mathrm{P}(\mathrm{B})>0$. We then write

$$
P(A \mid B)=\frac{P(A \cap B)}{P(B)},
$$

where $P(A \mid B)$ is called the conditional probability of $A$ under $B . \quad[P(A \mid B)$ is read:

"P of $\mathrm{A}$ under the condition $\left.\mathrm{B}^{\prime}.\right]$

A second definition which is connected with Definition 1 is the following.

\section{Definition 2}

Two events A and B are called independent, if

$$
\mathrm{P}(\mathrm{A} \cap \mathrm{B})=\mathrm{P}(\mathrm{A}) \mathrm{P}(\mathrm{B}) .
$$

This definition has an intuitive meaning. For, from Eq. (A.8) it follows that

$$
\mathrm{P}(\mathrm{A} \cap \mathrm{B})=\mathrm{P}(\mathrm{A} \mid \mathrm{B}) \mathrm{P}(\mathrm{B}),
$$

and if "A is independent of $B$ " we have $P(A \mid B)=P(A)$. We shall have examples for these two concepts later. 


\section{DISCRETE RANDOM VARIABLES}

Let $\delta$ be a random experiment. We said in the Introduction to the Appendix that, in general, it is possible to find a mathematical model for $\varepsilon$ of the form $\left(R_{n}, \mathscr{B}_{n}, P\right)$, where the corresponding random variable $\underline{x}$, which describes the outcome of $\delta$, takes values in $R_{n}$. This way we can identify the possible values of $\underline{X}$ with those of the outcome of $\mathcal{E}$.

Suppose now that the possible values of $\underline{x}$ are discrete values $\left(\underline{x}_{1}, \underline{x}_{2}, \ldots\right)$. This means that we have

$$
P\left(\underline{X}=\underline{x}_{i}\right)=p_{i}>0, \quad i=1,2, \ldots
$$

with

$$
\sum_{i=1}^{\infty} p_{i}=1
$$

and, for each $\underline{x} \neq \underline{x}_{i}, i=1,2, \ldots$, we have

$$
\mathrm{P}(\underline{\mathrm{X}}=\underline{\mathrm{x}})=0 \text {. }
$$

[Also, for each $B_{n} \in \mathcal{B}_{n}$ which does not contain any of the $\underline{x}_{i}$, we have $P\left(B_{n}\right)=0$. ]

In this case $\underline{X}$ is called a discrete random variable. Here, we must only know the $p_{i}$ and we can calculate the probability for all events $B_{n} \in \mathscr{B}_{n}$.

A few examples may clarify this concept a little.

i) $x=$ number observed when throwing a die. $x$ takes the values $\{1,2, \ldots, 6\}$.

ii) $x=$ number of defective items in a sample of size $n$ of a lot which contains $r \geq n$ defective items. $\mathrm{X}$ takes the values $\{0,1, \ldots, \mathrm{n}\}$.

iii) $\mathrm{x}=$ number of particles observed by a counter during a fixed time interval. $\mathrm{x}$ takes the values $\{0,1,2, \ldots\}$.

We said that the probability law or the distribution of a random variable $\underline{x}$ can be described by a (point) function $F(\underline{x})$, the distribution function of $\underline{x}$. This function can be defined by

$$
F(\underline{x})=P\left(X_{1} \leq x_{1}, \ldots, X_{n} \leq x_{n}\right)
$$

for each $\underline{x} \in R_{n}$, where the expression on the right-hand side of Eq. (A.14) is an abbreviation of $\mathrm{P}\left(\left\{\mathrm{X}_{1} \leq \mathrm{x}_{1}\right\} \cap\left\{\mathrm{X}_{2} \leq \mathrm{x}_{2}\right\} \cap \ldots \cap\left\{\mathrm{X}_{\mathrm{n}} \leq \mathrm{x}_{\mathrm{n}}\right\}\right)$. In other words $\mathrm{F}(\underline{\mathrm{x}})$ gives the probability that simultaneously values $x_{i} \leq x_{i}, i=1,2, \ldots, n$, are observed.

For a one-dimensional discrete random variable $\mathrm{X}, \mathrm{F}(\mathrm{x})$ is simply a step function given by

$$
\mathrm{F}(\mathrm{x})=\sum_{\mathrm{x}_{\mathrm{i}} \leq \mathrm{x}} \mathrm{p}_{\mathrm{i}}
$$

Figure A.2 shows the graph of example (i) with $p_{i}=1 / 6, i=1,2, \ldots, 6$. 


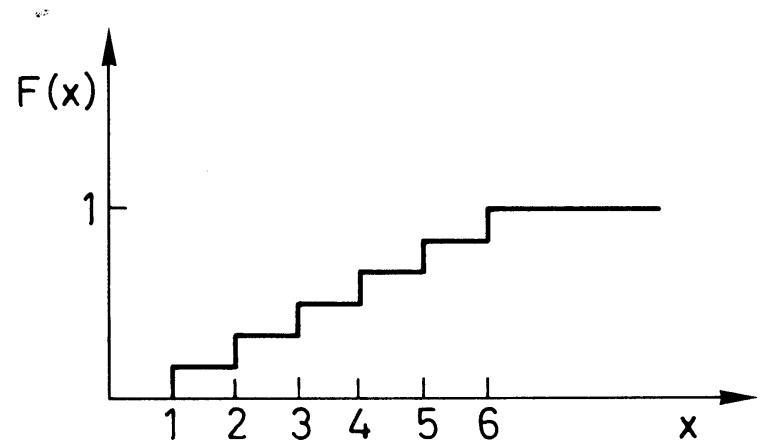

Fig. A.2 Distribution function of a die

If $\mathrm{F}(\mathrm{x})$ is known, the $\mathrm{p}_{\mathrm{i}}$ (in the discrete case) can be uniquely determined. Therefore the probability law is known either if the $p_{i}$ are known or if $F(x)$ is given.

We observe that, in general, we have to determine an infinite set of different parameters $p_{i}$ to know the probability law of a discrete random variable. Fortunately, in many applications it is sufficient to take special probability laws, in which the $p_{i}$ are known functions of only a few unknown parameters. This means that in these cases we only have to determine these unknown parameters.

We mention a few probability laws of discrete variables which occur very often in the applications.

a) Uniform distribution

$X$ takes $k$ different values $\left\{x_{1}, x_{2}, \ldots, x_{k}\right\}$.

$$
P\left(X=x_{i}\right)=\frac{1}{k}, \quad i=1,2, \ldots, k
$$

Example (i) has this distribution if the die is not biased.

b) Alternative distribution

$\mathrm{X}$ takes only two values, say, $\{0,1\}$.

$$
\mathrm{P}(\mathrm{X}=0)=1-\mathrm{p}, \quad \mathrm{P}(\mathrm{X}=1)=\mathrm{p}, \quad 0 \leq \mathrm{p} \leq 1 .
$$

This distribution depends on one unknown parameter p.

For the description of the next distribution which we want to consider, it is convenient to first introduce two more definitions.

\section{Definition 3}

The components $\left(\mathrm{x}_{1}, \ldots, \mathrm{x}_{\mathrm{n}}\right)$ of an $\mathrm{n}$-dimensional random variable are called independent, if

$$
F(\underline{x})=F_{1}\left(x_{1}\right) F_{2}\left(x_{2}\right) \ldots F_{n}\left(x_{n}\right) \text {, }
$$

where $F_{i}\left(x_{i}\right)$ are the distribution functions of the $x_{i}$. 


\section{Definition 4}

Let $x$ be a random variable and $x_{n}=\left(x_{1}, \ldots, x_{n}\right)$ an $n$-dimensional random variable, where all the components $x_{i}$ of $\underline{x}$ have the same distribution as $X$. We then call $x_{n}$ a random sample from $X$, if the $x_{i}$ are independent.

We now consider

c) Binomial distribution $B_{n}(\underline{r} ; p)$

Let $\mathrm{X}$ be a random variable like under (b) (alternative distribution). We take a random sample $\underline{X}_{n}$ from $X$ and define a new random variable

$$
\mathrm{N}=\sum_{\mathrm{i}=1}^{\mathrm{n}} \mathrm{X}_{\mathrm{i}}
$$

$\mathrm{N}$ takes values $\{0,1, \ldots, \mathrm{n}\}$. It can be shown that

$$
B_{n}(r ; p)=P(N=r)=\left(\begin{array}{l}
n \\
r
\end{array}\right) p^{r}(1-p)^{n-r}
$$

for $\mathrm{r}=0,1, \ldots, \mathrm{n}$. We say that $\mathrm{N}$ has a binomial distribution with parameter $\mathrm{p}$. The random variable $X i_{1}$.ample (ii) has a binomial distribution if the sample is a random sample.

d) Poisson distribution $\mathrm{P}(\mathrm{r} ; \mathrm{a})$

Let $\mathrm{R}$ be a random variable which takes the values $\{0,1,2, \ldots\}$. If

$$
P(r ; a)=P(R=r)=e^{-a} \frac{a^{r}}{r !}
$$

for $\mathrm{r}=0,1,2, \ldots$ and $\mathrm{a}>0, \mathrm{R}$ has a Poisson distribution with parameter $\mathrm{a}$.

It can be shown that the number $\mathrm{N}(\mathrm{t})$ of events which arrive independently and at random duri, it time interval of length $t$ has, under certain conditions, a Poisson distribution $\mathrm{P}(\mathrm{n} ; \lambda t)$. This means that, if on the average $\lambda$ events arrive during the time unit, the probability that $n=0,1, \ldots$ events arrive during the time $t$ is given by $\mathrm{P}(\mathrm{n} ; \lambda t)$.

\section{CONTINUOUS RANDOM VARIABLES}

We have characterized the discrete random variables by the fact that they take only a discrete set of different values. But there are also random variables which can take all values of a continuum. Such random variables are called continuous random variables. They are particularly characterized by the following property of their distribution function $\mathrm{F}(\underline{\mathrm{x}})$ : it is continuous and there exists a function $f(\underline{x}) \geq 0$ such that

$$
F(\underline{x})=\int_{-\infty}^{x_{1}} \cdots \int_{-\infty}^{x_{n}} f(\underline{x}) d x_{1} d x_{2} \ldots d x_{n}
$$

The function $f(\underline{x})$ is called the probability density function of the random variable $\underline{x}$. Equation (A.20) immediately leads to 


$$
\frac{\partial^{n} F(\underline{x})}{\partial x_{1} \ldots \partial x_{n}}=f(\underline{x})
$$

Therefore, if $F(\underline{x})$ is known, $f(\underline{x})$ is also known and vice versa, at least in principle.

This implies that the distribution of a continuous random variable $\underline{X}$ can also be described by the probability density function $f(\underline{x})$. In the applications it is of ten more convenient to work with the density function rather than to use the distribution function. It is also very simple to characterize the class of density functions, since every function $f(\underline{x})$ which satisfies

$$
\begin{gathered}
f(\underline{x}) \geq 0 \quad \text { for all } \quad \underline{x} \in R_{n} \\
\int_{R_{n}} f(\underline{x}) d \underline{x}=1
\end{gathered}
$$

is a probability density function. This fact immediately implies that there exist infinitely many probability density functions. Therefore, if we have a random experiment $\delta$ which leads to a continuous random variable $\underline{X}$, we have to search within this infinity of probability density functions to find the one which corresponds to $\delta$. At first glance it seems to be hopeless to have any idea what the correct function $f(\underline{x})$ may be. Fortunately, as in the case of the discrete random variables, there are typical density functions which occur very often in the applications and which are known up to some unknown constants. The following two examples may demonstrate that. More examples are given in Chapter I.

a) Uniform distribution

$\mathrm{X}$ takes values in an interval $(\mathrm{a}, \mathrm{b})$ and its probability density function is given by

$$
f(x)=\left\{\begin{array}{cl}
\frac{1}{b-a}, & \text { for } a<x<b, \\
0, & \text { elsewhere } .
\end{array}\right.
$$

If $\mathrm{a}=0, \mathrm{~b}=1$, this distribution is particularly important for Monte Carlo methods, which are not discussed here but can be studied for instance in a book by Hammersley and Handscomb ${ }^{10}$ ).

b) Normal distribution

$\mathrm{X}$ takes values between $-\infty$ and $+\infty$ and its probability density function is given by

$$
f(x)=\frac{1}{\sqrt{2 \pi} \sigma} \exp \left[-\frac{(x-\mu)^{2}}{2 \sigma^{2}}\right] \text {, }
$$

where $\sigma>0$ and $-\infty<\mu<\infty$ are parameters which have to be chosen in a proper way for each particular random experiment $\delta$, which we can assume has a normal distribution. How to find at least approximate values for $\mu$ and $\sigma$ is a statistical problem and will be discussed later. The parameters $\mu$ and $\sigma$ have an intuitive meaning as we shall see soon.

The normal distribution can be applied to many practical problems. The reason why this is true will be seen later. 


\section{MATHEMATICAL EXPECTATION}

The concept of mathematical expectation is based on the following idea.

Suppose there is a die having probability $p_{i}$ that the figure $x_{i}, i=1, \ldots, 6$, is thrown. We consider the following game. A player gains $g\left(x_{i}\right)$ dollars (he has to pay, if $g$ is negative) when he throws $x_{i}$. If he plays the game for a long time what will be his expected gain? Since he will receive $g\left(x_{i}\right)$ with probability $p_{i}$ for $i=1, \ldots, 6$, it seems to be reasonable to define the expected or average gain by

$$
E[g]=\sum_{i=1}^{6} g\left(x_{i}\right) p_{i}
$$

This example motivates the following definition.

\section{Definition 5}

Let $\underline{X}$ be a random variable with distribution function $F(\underline{x})$, and let $g(\underline{x})$ be a real or complex function such that

$$
E[g(\underline{x})]=\int_{R_{n}} g(\underline{x}) d F(\underline{x})
$$

exists. Then $E[g(\underline{X})]$ is called the mathematical expectation of $g(\underline{X})$.

In particular, we have for Eq. (A.27) the expressions

$$
E[g(\underline{x})]=\sum_{i=1}^{\infty} g\left(x_{i}\right) p_{i}
$$

in the case of a discrete random variable $\underline{X}$, and

$$
E[g(\underline{x})]=\int_{R_{n}} g(\underline{x}) f(\underline{x}) d \underline{x}
$$

in the case of a continuous random variable with density function $f(\underline{x})$.

From Definition 5 it immediately follows that the mathematical expectation is a linear operator, i.e.

$$
E\left[\alpha g_{1}(\underline{X})+\beta g_{2}(\underline{X})\right]=\alpha E\left[g_{1}(\underline{X})\right]+\beta E\left[g_{2}(\underline{X})\right] \text {, }
$$

where $\alpha$ and $\beta$ are constants, and $g_{1}$ and $g_{2}$ are suitable functions.

The choice of particular functions $g(\underline{x})$ in Definition 5 leads to special constants associated with the distribution of $\underline{x}$. For a one-dimensional random variable the following examples occur very often in the applications and they even have special names:

$$
\mu_{\mathrm{n}}^{\prime}=E\left[\mathrm{X}^{\mathrm{n}}\right], \quad\left(\mathrm{n}^{\mathrm{th}} \text { moment of } \mathrm{X}\right),
$$




$$
\begin{gathered}
\mu_{1}^{\prime}=\mu_{X}=E\left[X^{1}\right], \quad(\text { mean value of } X), \\
\mu_{n}=E\left[\left(X-\mu_{X}\right)^{n}\right], \quad\left(\text { central } n^{\text {th }} \text { moment of } X\right), \\
\operatorname{var}(X)=\sigma_{X}^{2}=E\left[\left(X-\mu_{X}\right)^{2}\right], \quad(\text { variance of } X), \\
\left.\operatorname{cov}\left(X_{1}, X_{2}\right)=\sigma_{X_{1} X_{2}}=E\left[\left(X_{1}-\mu_{X_{1}}\right)\left(X_{2}-\mu_{X_{2}}\right)\right], \quad \text { (covariance of } X_{1} \text { and } X_{2}\right) .
\end{gathered}
$$

The importance of these and other mathematical expectations lies in the following fact. Very often in the applications we are not able to determine the whole distribution function $F(x)$ or, in the continuous case, the density function $f(x)$ of a random variable, because we may not have enough information. But if we have a random sample $\left(X_{1}, X_{2}, \ldots, X_{n}\right)$ from $X$, it is shown in statistics that we can easily obtain estimates for the expected values. These are simply given by the expression

$$
\hat{E}_{n}[g(x)]=\frac{1}{n} \sum_{i=1}^{n} g\left(X_{i}\right),
$$

Now, the expressions (A.36) give us at least some information about the distribution which underlies the corresponding random variable $X$. In many practical cases, expressions of type (A.36) even determine approximately the whole distribution.

Let us discuss the following example. Suppose we have a random variable $X$, of which we know that it has a normal distribution, but where we do not know the parameters $\mu$ and $\sigma^{2}$. In addition, we assume that we have a random sample $\left(X_{1}, X_{2}, \ldots, X_{n}\right)$ of size $n$ from $\mathrm{X}$. How can we determine approximately the distribution of $\mathrm{X}$ ?

Using the density function of the normal distribution given by Eq. (A.25), it can be shown that

$$
\begin{gathered}
E[x]=\int_{-\infty}^{\infty} x f(x) d x=\mu, \\
E\left[(x-\mu)^{2}\right]=\int_{-\infty}^{\infty}(x-\mu)^{2} f(x) d x=\sigma^{2} .
\end{gathered}
$$

These two formulae show us that the unknown parameters of the normal distribution are the mean and the variance of $x$. Before we continue, let us briefly discuss the intuitive meaning of these two constants. The parameter $\mu$ is the value around which all observations vary when we take samples from $X$. The parameter $\sigma^{2}$ gives a measure of the spread of the sample points around $\mu$. If $\sigma^{2}$ is small, the points are mostly close to $\mu$, if $\sigma^{2}$ is large they may be spread all over the axis. This behaviour is also indicated by the shape of $f(x)$ given in Fig. A.3. 


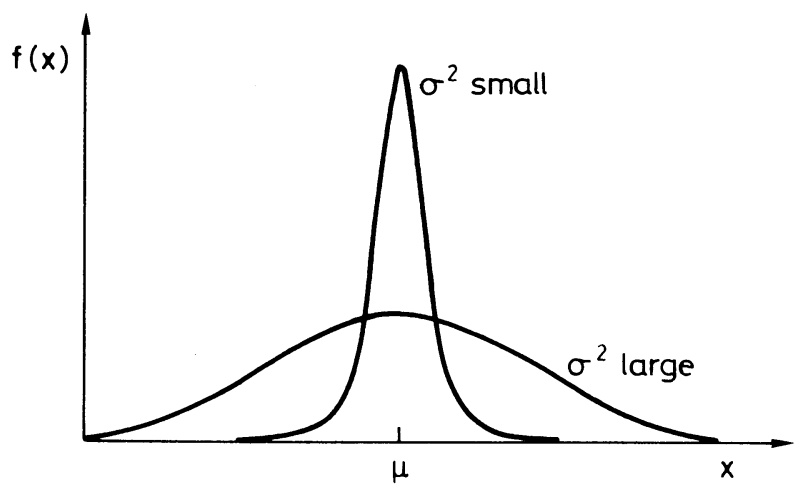

Fig. A.3 Density of the normal distribution

Let us come back to the approximation of $\mu$ and $\sigma^{2}$. Using Eq. (A.36) with $g(x)=x$, we immediately find as an approximation to $\mu$ :

$$
\hat{\mu}=\frac{1}{n} \sum_{i=1}^{n} x_{i} .
$$

For $\sigma^{2}$, the problem is a bit more complicated, since here the function $g(x)=(x-\mu)^{2}$, which is dependent on the unknown value $\mu$. However in statistics it is shown that the expression

$$
\hat{\sigma}^{2}=\frac{1}{n} \sum_{i=1}^{n}\left(x_{i}-\hat{\mu}\right)^{2}
$$

is a "good" estimate for $\sigma^{2}$, at least for large $n$. [For smaller $\mathrm{n}$ it is preferable to take $1 /(n-1)$ instead of $1 / \mathrm{n}$ as the factor of the sum in Eq. (A.40).] This way we have found a means to determine approximately the total distribution of our random variable $X$. How good is the approximation? This is a question which is handled in statistics. But it will not be discussed here. It can be found in the literature on mathematical statistics under the heading "Estimation of unknown parameters" [see, for example, Guttman and Wilks ${ }^{12}$ ) and Eadie et al. 17)].

\section{LAW OF LARGE NUMBERS. CENTRAL LIMIT THEOREM}

Let us recall what we said in the Introduction to the Appendix when we motivated the definition of the probability of an event. There we said that it can be shown that the relative frequency $Q_{n}$ of an event tends to the probability of the event with an increasing number $\mathrm{n}$ of trials. This means that the intuitive concept of probability of an event (limit of the relative frequency) and the axiomatic concept (probability axioms) coincide in some sense. We say "in some sense" because we have to define the meaning of " $Q_{n}$ tends to $\mathrm{p}^{\prime \prime}$.

This is one problem we want to discuss in this section. A second one to be treated here is the statement from Section 4 of the Appendix that the normal distribution can be applied to many practical problems. 
For convenience, we confine the discussion to one-dimensional random variables. Suppose we have an infinite sequence $x_{1}, X_{2}, \ldots$ of random variables with the corresponding distribution functions $F_{1}(x), F_{2}(x), \ldots$. We then make the following definition.

\section{Definition 6}

We say that the sequence $x_{1}, x_{2}, \ldots$ converges in distribution to the random variable $X$ with the distribution function $F(x)$, if for each point where $F(x)$ is continuous, we have

$$
\lim _{n \rightarrow \infty} F_{n}(x)=F(x)
$$

[This gives one definition of a concept of convergence of random variables. There are others, but they are more difficult to understand. For these concepts the interested reader may, for instance, refer to Hogg and Craig $\left.^{18)}.\right]$

Let us take a simple example. A random variable which takes only one value, say $\mu$, is called a degenerated random variable. In this case we have $P(X=\mu)=1$ and $P(X=x)=0$ for all $\mathrm{x} \neq \mu$. The corresponding distribution function $\mathrm{F}(\mathrm{x})$ is given by

$$
F(x)= \begin{cases}0, & x<\mu, \\ 1, & x \geq \mu .\end{cases}
$$

For a degenerated random variable which takes the value $\mu$ we write shortly $\mu$.

Let us now take a sequence $\left\{\mathrm{x}_{\mathrm{n}}\right\}$ of normally distributed random variables with mean value $\mu$ and corresponding variances $\sigma^{2}=1 / \mathrm{n}$. We can then show that the corresponding distribution functions $\mathrm{F}_{\mathrm{n}}(\mathrm{x})$ converge to Eqs. (A.42). This is easily done by using formula (A.25). The result is intuitively clear when we remember the meaning of the variance which in the considered case tends to zero.

We now formulate a theorem without proof which is very important for statistics and which also enables us to show that $Q_{n}$ tends in distribution to $p$.

\section{Theorem 1}

Let $\left\{x_{n}\right\}$ be a sequence of independent random variables which all have the same distribution as some random variable $\mathrm{X}$. In addition, let $\mathrm{g}(\mathrm{X})$ be a function such that $E[|g(X)|]<\infty$. Then

$$
\hat{\mathrm{E}}_{\mathrm{n}}[\mathrm{g}(\mathrm{X})]=\frac{1}{\mathrm{n}} \sum_{\mathrm{i}=1}^{\mathrm{n}} \mathrm{g}\left(\mathrm{x}_{\mathrm{i}}\right)
$$

converges in distribution to $\mathrm{E}[\mathrm{g}(\mathrm{X})]$.

This theorem gives an explanation why Eq. (A.36) was used as an approximation to $E[g(X)]$. We use it to prove that the relative frequency $Q_{n}$ tends in distribution to the probability p. The latter statement is one version of what is generally called the law of large numbers. 
Let $\mathrm{E}$ be an event of a random experiment $\delta$. We define a random variable $\mathrm{X}$ which takes the values 0 and 1 :

$$
X= \begin{cases}1, & \text { if } E \text { occurs, when } \delta \text { is performed; } \\ 0, & \text { if } \bar{E} \text { occurs, when } \delta \text { is performed. }\end{cases}
$$

We then have

$$
\mathrm{p}=\mathrm{p}(\mathrm{E})=\mathrm{P}(\mathrm{X}=1)
$$

We repeat $\delta$ independently $n$ times and obtain for the relative frequency $Q_{n}$ of $E$ :

$$
\mathrm{Q}_{\mathrm{n}}=\frac{1}{\mathrm{n}} \sum_{\mathrm{i}=1}^{\mathrm{n}} \mathrm{X}_{\mathrm{i}}
$$

where $X_{i}$ are the observed values of $X$. Since $E[|X|]=p \cdot 1+(1-p) \cdot 0=p<\infty$, the assumption of Theorem 1 is satisfied and it follows that $Q_{n} \rightarrow E[X]=p$ in distribution, proving the law of large numbers.

We now turn to the question of the importance of the normal distribution. It is demonstrated by the following theorem.

\section{Theorem 2}

Let $\left\{\mathrm{X}_{\mathrm{n}}\right\}$ be a sequence of independent random variables which all have the same distribution. Suppose that $\mu=E\left[X_{n}\right], \sigma^{2}=\operatorname{var}\left(X_{n}\right)$ exist. Then

$$
z_{n}=\frac{1}{\sqrt{n}} \sum_{i=1}^{n} \frac{x_{i}-\mu}{\sigma}
$$

converges in distribution to a random variable $\mathrm{Z}$ which has a standardized normal distribution (i.e. with mean zero and variance one).

Since the proof of Theorem 2 is too complicated we shall skip it here.

Theorem 2 can be generalized to the case where the $x_{i}$ do not have the same distribution. It is known in literature under the name "Central limit theorem". We may interpret it in the following way. Whenever a random variable (like $Z$ in Theorem 2) can be considered as a sum of many independent random variables it is approximately normally distributed. In other words, if a random phenomenon can be considered to be the result of many other (independent) random phenomena, it tends to have a normal distribution.

\section{CONDITIONAL DISTRIBUTIONS}

From Definition 1 in Section 2 of the Appendix we already know the definition of the conditional probability of two events. We now want to define what a conditional distribution is. To first obtain an intuitive feeling for this concept we consider the following example. Suppose we have two random variables $U$ and $V$ which may, for instance, describe two velocity components in a turbulent flow at a particular point and at a particular time. We then can, for example, ask the question what the probability is that U is less than or 
equal to some given $\mathrm{u}$, under the condition that we know that $\mathrm{v}=\mathrm{v}$, where $\mathrm{v}$ is a given value. In this case we call $U$ a conditional random variable. The variable $U$ then is still a random variable, but if $U$ and $V$ are not independent, the conditional variable $U / V$ (" $U$ under the condition $V^{\prime \prime}$ ) has another distribution which is called the conditional distribution. Particularly when we assume that the conditional random variable is continuous, it must have a probability density which is denoted by $f(u \mid v)$ and which is called a conditional probability density function. It can be shown that we have

$$
f(u \mid v)=\frac{f(u, v)}{f_{2}(v)}
$$

where $f(u, v)$ is the density of the two-dimensional random variable $(U, v)$ and $f_{2}(v)$ is the density of $\mathrm{V}$.

The concept of conditional distributions is very important for practical problems. Applications of it are given in the main paper on reliability.

\section{SUMS OF INDEPENDENT RANDOM VARIABLES}

In reliability theory and in many other applications of probability theory it often occurs that we have a sum

$$
\mathrm{T}_{\mathrm{n}}=\sum_{\mathrm{i}=0}^{\mathrm{n}} \mathrm{x}_{\mathrm{i}}
$$

of independent random variables $x_{i}$ having distribution functions $G_{i}\left(x_{i}\right)$ and densities $g_{i}\left(x_{i}\right)$ for $i=0,1, \ldots, n$. The quantity $T_{n}$ is then itself a random variable and it is important to find its distribution function $F_{n}\left(t_{n}\right)$ supposing that the $G_{i}\left(x_{i}\right)$ or the $g_{i}\left(x_{i}\right)$ are al1 known.

In order to find the distribution function of $\mathrm{T}_{\mathrm{n}}$ a recurrence relation can be derived. Let

$$
\mathrm{T}_{j}=\sum_{\mathrm{i}=0}^{\mathrm{j}} \mathrm{x}_{\mathrm{i}}
$$

for $j=1, \ldots, n$. In textbooks on probability and statistics (see Ref. 18) it is shown that for $j=1$ we have

$$
F_{1}\left(t_{1}\right)=\int_{-\infty}^{\infty} G_{1}\left(t_{1}-t_{0}\right) d F_{0}\left(t_{0}\right),
$$

where $F_{0}\left(t_{0}\right)=G_{0}\left(t_{0}\right)$. The integral in Eq. (A.46) is called a convolution integral of $F_{0}$ and $G_{1}$. Using the probability density functions $f_{j}\left(t_{j}\right)$ and $g_{i}\left(x_{i}\right)$ corresponding to $T_{j}$ and $x_{i}$, respectively, we obtain from Eq. (A.46) the formulae

$$
F_{1}\left(t_{1}\right)=\int_{-\infty}^{\infty} G_{1}\left(t_{1}-t_{0}\right) f_{0}\left(t_{0}\right) d t_{a},
$$




$$
f_{1}\left(t_{1}\right)=\int_{-\infty}^{\infty} g_{1}\left(t_{1}-t_{0}\right) f_{0}\left(t_{0}\right) d t_{0}
$$

It is now easy to see that we have

$$
\begin{aligned}
& F_{j}\left(t_{j}\right)=\int_{-\infty}^{\infty} G_{j}\left(t_{j}-t_{j-1}\right) d F_{j-1}\left(t_{j-1}\right), \\
& F_{j}\left(t_{j}\right)=\int_{-\infty}^{\infty} G_{j}\left(t_{j}-t_{j-1}\right) f_{j-1}\left(t_{j-1}\right) d t_{j-1} \\
& f_{j}\left(t_{j}\right)=\int_{-\infty}^{\infty} g_{j}\left(t_{j}-t_{j-1}\right) f_{j-1}\left(t_{j-1}\right) d t_{j-1}
\end{aligned}
$$

for $j=1,2, \ldots, n$ and $n=1,2, \ldots$. 


\section{REFERENCES}

1) K.B. Brink, T. Conrady, H.J. Keller and R. Keppeler, Technische Wartbarkeit und Zuverlässigkeit, ihr Einfluss auf den wirtschaftlichen Betrieb, Lehrgangshandbuch, (VDI-Bildungswerk, Düsseldorf, 1973).

2) B.A. Kozlov and I.A. Ushakov, Reliability handbook (Holt, Rinehart and Winston, New York, 1970).

3) G.W.A. Dummer and R.C. Winton, An elementary guide to reliability (Pergamon, Oxford, 1968).

4) R.H. Myers, K.L. Wong and H.M. Gordy, Reliability engineering for electronic systems (John Wiley, New York, 1964).

5) W. Ireson, Reliability handbook (McGraw-Hill, New York, 1966).

6) R.E. Barlow and F. Proschan, Mathematical theory of reliability (John Wiley, New York, 1967).

7) P. Chapouille and R. de Pazzis, Fiabilité des systèmes (Masson, Paris, 1968).

8) G. Doetsch, Handbuch der Laplace-Transformation (Birkhäuser, Base1, 1950-1955), Vo1s. $1-3$.

9) R.V. Churchil1, Operational mathematics (McGraw-Hil1, New York, 1972).

10) J.M. Hammersley and D.C. Handscomb, Monte Carlo methods (Methuen, London, 1964).

11) R.B. Cooper, Introduction to Queuing Theory (Macmillan, New York, 1972) •

12) I. Guttman and S.S. Wilks, Introductory engineering statistics (John Wiley, New York, 1971).

13) S. Dworatschek and H. Dönike, Wirtschaftlichkeitsanalyse von Informationssystemen, (de Gruyter, Berlin, 1972).

14) C. Zangemeister, Nutzwertanalyse in der Systemtechnik (Wittemann, München, 1973).

15) C. McMillan, Jr., Mathematical programming (John Wiley, New York, 1970) •

16) H. Müller-Merbach, Operations research (Franz Vahlen, München, 1971).

17) W.T. Eadie, D. Drijard, F. James, M. Roos and B. Sadoulet, Statistical methods in experimental physics (North-Holland, Amsterdam, 1971).

18) R.V. Hogg and A.T. Craig, Introduction to mathematical statistics Macmillan, New York, 1967). 